\title{
The Stabilized Cation Pool Method: Metal- and Oxidant-Free Benzylic C-H/Aromatic C-H Cross-Coupling
}

\author{
Ryutaro Hayashi, Akihiro Shimizu, and Jun-ichi Yoshida* \\ Department of Synthetic Chemistry and Biological Chemistry, Graduate School of Engineering, \\ Kyoto University, Kyotodaigaku-katsura Nishikyo-ku, Kyoto 615-8510, Japan. \\ E-mail: yoshida@ @bchem.kyoto-u.ac.jp
}

1. General

$\mathrm{S} 2$

2. Synthesis of Sulfilimines

S3

3. Reaction of 4-Methoxybenzyl Bromide with Benzofuran

S4

4. Generation and Observation of Benzylaminosulfonium Ion 3a.

S4-S5

5. Reaction of 3 with Benzofuran.

S5-S10

6. Oxidation Potentials

S11

7. Reactions of 3a with Nucleophiles.

S12-S14

8. Synthesis of Inhibitor of PTPase

S15-S17

9. Reference

$\mathrm{S} 18$

10. NMR Spectra

S19-S55 


\section{General}

${ }^{1} \mathrm{H}$ and ${ }^{13} \mathrm{C}$ NMR spectra were recorded in $\mathrm{CDCl}_{3}$ on a Varian MERCURY plus-400 $\left({ }^{1} \mathrm{H} 400 \mathrm{MHz},{ }^{13} \mathrm{C}\right.$ $100 \mathrm{MHz}$ ) spectrometer with tetramethylsilane as an internal standard unless otherwise noted. Mass spectra were obtained on a JEOL EXACTIVE (ESI and APCI) mass spectrometer and a JEOL JMS-SX102A mass spectrometer (EI). GC analysis was performed on a SHIMADZU GC-2014 gas chromatograph equipped with a flame ionization detector using a fused silica capillary column (column, CBP1; $0.22 \mathrm{~mm}$ x $25 \mathrm{~m}$ ). Merck precoated silica gel F254 plates (thickness $0.25 \mathrm{~mm}$ ) were used for thin-layer chromatography (TLC) analysis. Flash chromatography was carried out using Kanto Chem. Co., Silica Gel N (spherical, neutral, 40-100 $\mu \mathrm{m}$ ). Preparative gel permeation chromatography (GPC) was performed on a Japan Analytical Industry LC-918. All reactions were carried out under argon atmosphere unless otherwise noted. Unless otherwise noted, all materials were obtained from commercial suppliers and were used without further purification.

The anodic oxidation was carried out using an H-type divided cell (4G glass filter) equipped with an anode made of carbon strings (Nippon Carbon GF-20-P21E, ca. $160 \mathrm{mg}$ for $0.20 \mathrm{mmol}$ scale, dried at $300{ }^{\circ} \mathrm{C} / 1 \mathrm{mmHg}$ for $3 \mathrm{~h}$ before use) and a platinum plate cathode $(10 \mathrm{~mm} \times 10 \mathrm{~mm})$. Dichloromethane was washed with water, distilled from $\mathrm{P}_{2} \mathrm{O}_{5}$, redistilled from dried $\mathrm{K}_{2} \mathrm{CO}_{3}$ to remove a trace amount of acid, and stored over molecular sieves $4 \mathrm{~A}$. $\mathrm{NaB}\left(\mathrm{C}_{6} \mathrm{~F}_{5}\right)_{4}$ was provided from Nippon Shokubai Co. as a precursor of $\mathrm{Bu}_{4} \mathrm{NB}\left(\mathrm{C}_{6} \mathrm{~F}_{5}\right)_{4}$. Bu $\mathrm{Bu}_{4} \mathrm{NB}\left(\mathrm{C}_{6} \mathrm{~F}_{5}\right)_{4}$ was synthesized according to the reported literature ${ }^{1}$.

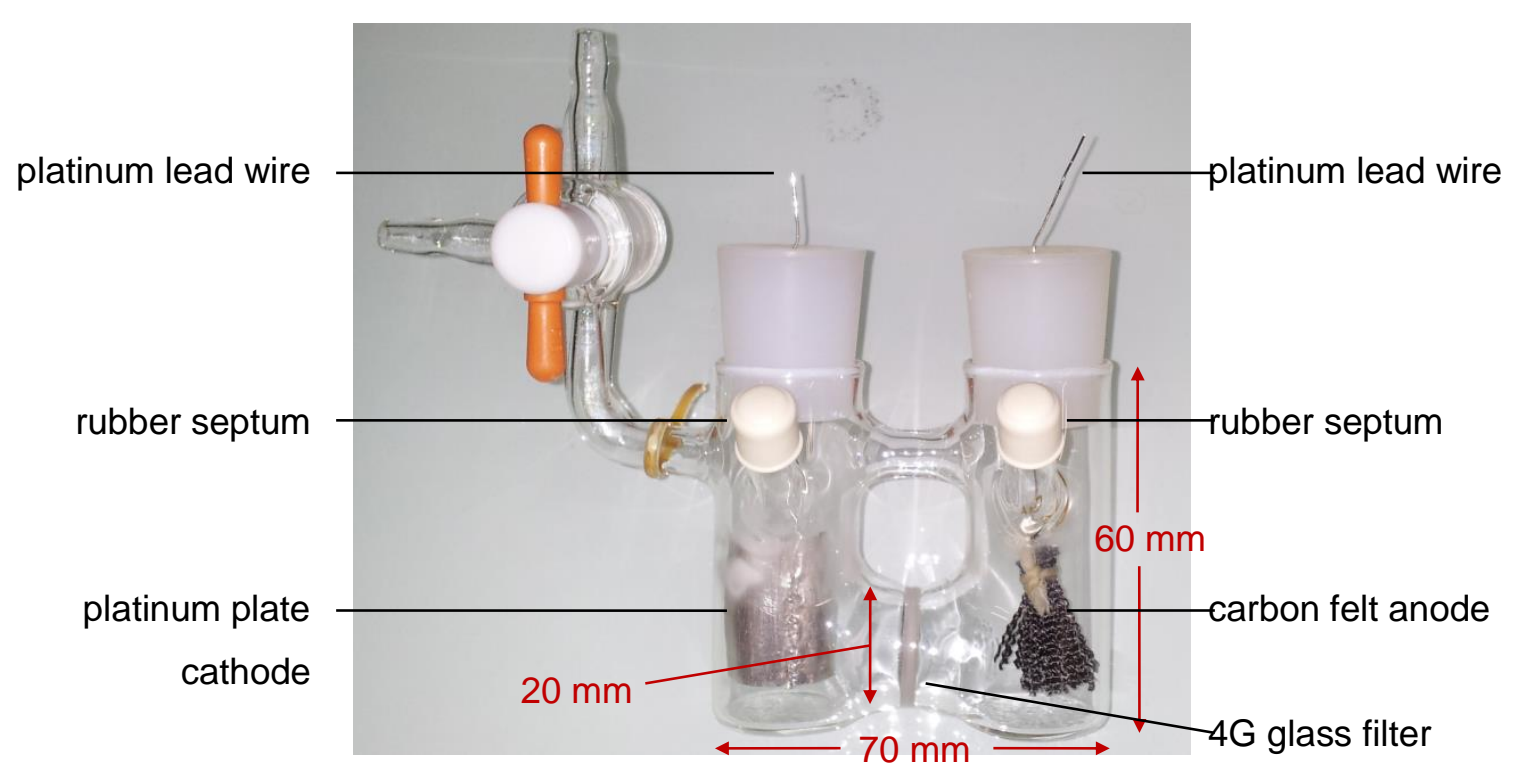

Figure S1. An H-type divided cell for electrolysis. 


\section{Synthesis of Sulfilimines}

Synthesis of $S, S$-Dimethyl- $N$-(4-methylphenylsulfonyl)sulfilimine (2a) ${ }^{2}$

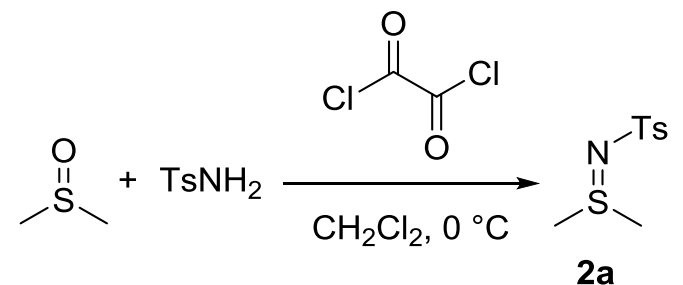

To a round-bottom flask were added DMSO ( $5 \mathrm{~mL}, 70 \mathrm{mmol}), p$-toluenesulfonamide $(1.7 \mathrm{~g}, 10 \mathrm{mmol})$, and $\mathrm{CH}_{2} \mathrm{Cl}_{2}(40 \mathrm{~mL})$. A solution of oxalyl chloride $(1.9 \mathrm{~mL}, 22 \mathrm{mmol})$ in $\mathrm{CH}_{2} \mathrm{Cl}_{2}(5 \mathrm{~mL})$ was added dropwise at $0{ }^{\circ} \mathrm{C}$ over $3 \mathrm{~min}$. After being stirred at $0{ }^{\circ} \mathrm{C}$ for $2 \mathrm{~h}, \mathrm{Et}_{3} \mathrm{~N}(8.5 \mathrm{~mL})$ was added to the mixture. The mixture was washed with $1 \mathrm{M} \mathrm{NaOH}$ aq, $\mathrm{H}_{2} \mathrm{O}$, and brine, dried over $\mathrm{Na}_{2} \mathrm{SO}_{4}$, and concentrated in vacuum. The residue was purified by recrystallization from $\mathrm{CH}_{2} \mathrm{Cl}_{2}$ to give $S, S$-dimethyl- $N$-(4-methylphenylsulfonyl)sulfilimine (2a) in $76 \%$ yield (1.76 g, $7.6 \mathrm{mmol})$. TLC $\mathrm{R}_{f} 0.08$ (EtOAc); ${ }^{1} \mathrm{H}$ NMR (400 MHz, $\left.\mathrm{CDCl}_{3}\right) \delta 2.40$ (s, 3H), 2.69 (s, 6H), 7.25 (d, $\left.J=7.9 \mathrm{~Hz}, 2 \mathrm{H}\right), 7.78$ (d, $J=8.3$ $\mathrm{Hz}, 2 \mathrm{H}) ;{ }^{13} \mathrm{C}$ NMR $\left(100 \mathrm{MHz}, \mathrm{CDCl}_{3}\right) \delta 21.4,36.0,126.2,129.3,141.2,141.8$; HRMS (ESI) calcd for $\mathrm{C}_{9} \mathrm{H}_{13} \mathrm{NO}_{2} \mathrm{~S}_{2}\left(\mathrm{M}+\mathrm{H}^{+}\right): 232.0460$, found: 232.0457 .

\section{Synthesis of $S, S$-Diphenyl- $N$-(4-methylphenylsulfonyl)sulfilimine $2 \mathrm{~b}^{3}$}

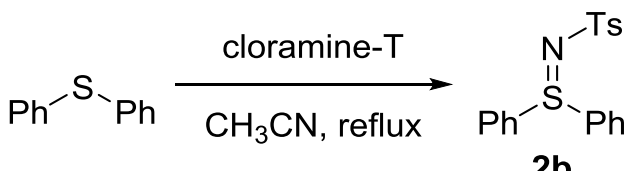

2b

To a round-bottom flask were added phenyl sulfide $(13 \mathrm{~mL}, 80 \mathrm{mmol}$ ), cloramine-T (trihydrate, $25 \mathrm{~g}, 110$ mmol), and $\mathrm{CH}_{3} \mathrm{CN}$ (200 mL). The mixture was refluxed for $3 \mathrm{~h} . \mathrm{CH}_{2} \mathrm{Cl}_{2}(300 \mathrm{~mL})$ was added to the solution and the precipitate was removed by filtration. The filtrate was concentrated under vacuum and the residue was purified by recrystallization from EtOAc and hexane to give $\boldsymbol{S}, \boldsymbol{S}$-diphenyl- $\boldsymbol{N}$-(4-methylphenylsulfonyl)sulfilimine (2b) in $66 \%$ yield $(19 \mathrm{~g}, 53 \mathrm{mmol})$. TLC $\mathrm{R}_{f} 0.36$ (hexane/EtOAc 1:1); ${ }^{1} \mathrm{H}$ NMR (400 MHz, $\left.\mathrm{CDCl}_{3}\right) \delta 2.24$ (s, 3H), $7.14(\mathrm{~d}, J=7.9 \mathrm{~Hz}, 2 \mathrm{H}), 7.42-7.48(\mathrm{~m}$, $4 \mathrm{H}), 7.48-7.53(\mathrm{~m}, 2 \mathrm{H}), 7.60-7.64(\mathrm{~m}, 4 \mathrm{H}), 7.75(\mathrm{~d}, J=8.3 \mathrm{~Hz}, 2 \mathrm{H}) ;{ }^{13} \mathrm{C} \mathrm{NMR}\left(100 \mathrm{MHz}, \mathrm{CDCl}_{3}\right) \delta 21.4$, $126.3,127.2,129.1,129.8,132.2,136.6,141.4,141.6$; HRMS (ESI) calcd for $\mathrm{C}_{19} \mathrm{H}_{18} \mathrm{NO}_{2} \mathrm{~S}_{2}\left(\mathrm{M}+\mathrm{H}^{+}\right)$: 356.0773, found: 356.0766 . 


\section{Reaction of 4-Methoxybenzyl bromide with benzofuran}

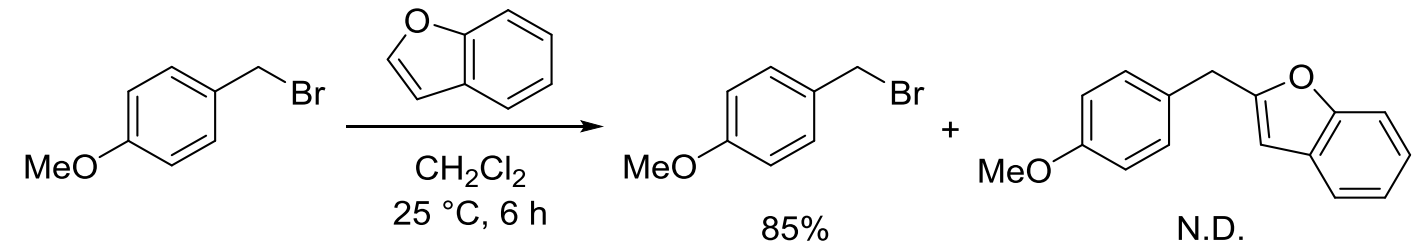

To a round-bottom flask were added 4-methoxybenzyl bromide (19.5 mg, $0.097 \mathrm{mmol}$ ), benzofuran (55 $\mu \mathrm{L}, 0.51 \mathrm{mmol})$, and $\mathrm{CH}_{2} \mathrm{Cl}_{2}(10 \mathrm{~mL})$. The mixture was stirred at $25^{\circ} \mathrm{C}$ for $6 \mathrm{~h}$. The solvent was removed under reduced pressure. The GC analysis using hexadecane as an internal standard indicated that 2-(4-methoxybenzyl)-benzofuran was not produced and that 4-methoxybenzyl bromide was recovered in $85 \%$ yield.

\section{Generation and Observation of $p$-Methoxybenzylaminosulfonium Ion (3a)}

\section{Observation of 3a by NMR Spectroscopy}

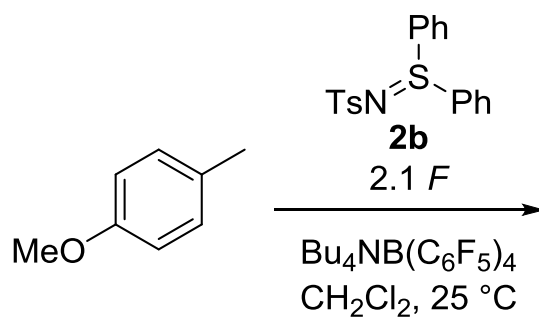

$1 \mathrm{a}$<smiles>COc1ccc(CN([As])[Sb](c2ccccc2)c2ccccc2)cc1</smiles>

$3 a$

In the anodic chamber were placed 4-methoxytoluene (1a) $(24.4 \mathrm{mg}, 0.200 \mathrm{mmol})$,

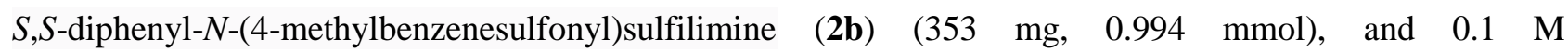
$\mathrm{Bu}_{4} \mathrm{NB}\left(\mathrm{C}_{6} \mathrm{~F}_{5}\right)_{4} / \mathrm{CH}_{2} \mathrm{Cl}_{2}(10 \mathrm{~mL})$. In the cathodic chamber were placed $\mathrm{TfOH}(60 \mu \mathrm{L}, 0.68 \mathrm{mmol})$ and $0.1 \mathrm{M}$ $\mathrm{Bu}_{4} \mathrm{NB}\left(\mathrm{C}_{6} \mathrm{~F}_{5}\right)_{4} / \mathrm{CH}_{2} \mathrm{Cl}(10 \mathrm{~mL})$. The constant current electrolysis $(8.0 \mathrm{~mA})$ was carried out at $25{ }^{\circ} \mathrm{C}$ with magnetic stirring until $2.1 F$ of electricity was consumed. The solution in the anodic chamber was collected and the solvent was removed under reduced pressure. The crude product was dissolved in $\mathrm{CDCl}_{3}$ and dried over molecular sieves $4 \mathrm{~A}$. The solution was analyzed by NMR spectroscopy. ${ }^{1} \mathrm{H}$ NMR $\left(400 \mathrm{MHz}, \mathrm{CDCl}_{3}\right) \delta$ $2.43(\mathrm{~s}, 3 \mathrm{H}), 3.73$ (s, 3H), 4.75 (s, 2H), $6.68(\mathrm{~d}, J=8.8 \mathrm{~Hz}, 2 \mathrm{H}), 6.78(\mathrm{~d}, J=8.8 \mathrm{~Hz}, 2 \mathrm{H}), 7.30$ (d, $J=8.3 \mathrm{~Hz}$, 2H), $7.39(\mathrm{~d}, J=8.3 \mathrm{~Hz}, 2 \mathrm{H}), 7.50-7.54(\mathrm{~m}, 4 \mathrm{H}), 7.62-7.65(\mathrm{~m}, 4 \mathrm{H}), 7.82(\mathrm{t}, J=7.5 \mathrm{~Hz}, 2 \mathrm{H})$. 


\section{Observation of 3a by Mass Spectroscopy}

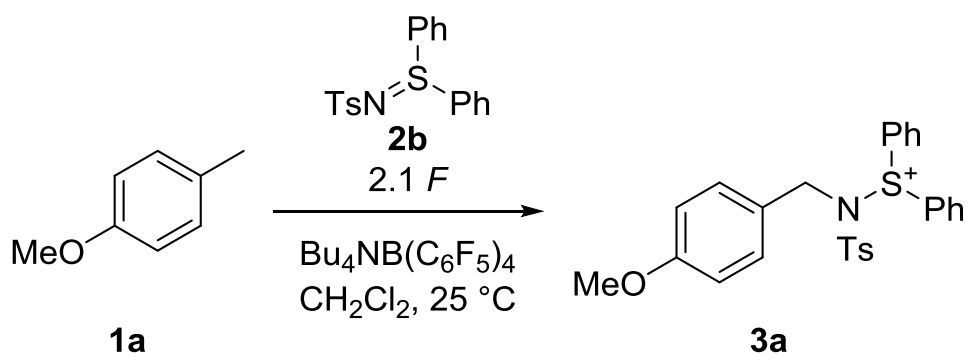

In the anodic chamber were placed 4-methoxytoluene (1a) $(23.6 \mathrm{mg}, 0.193 \mathrm{mmol})$, $S, S$-diphenyl- $N$-(4-methylbenzenesulfonyl)sulfilimine $\quad(\mathbf{2 b}) \quad\left(\begin{array}{llllll}348 & \mathrm{mg}, & 0.980 & \mathrm{mmol}\end{array}\right), \quad$ and $\quad 0.1 \quad \mathrm{M}$ $\mathrm{Bu}_{4} \mathrm{NB}\left(\mathrm{C}_{6} \mathrm{~F}_{5}\right)_{4} / \mathrm{CH}_{2} \mathrm{Cl}_{2}(10 \mathrm{~mL})$. In the cathodic chamber were placed $\mathrm{TfOH}(60 \mu \mathrm{L}, 0.68 \mathrm{mmol})$ and $0.1 \mathrm{M}$ $\mathrm{Bu}_{4} \mathrm{NB}\left(\mathrm{C}_{6} \mathrm{~F}_{5}\right)_{4} / \mathrm{CH}_{2} \mathrm{Cl}(10 \mathrm{~mL})$. The constant current electrolysis $(8.0 \mathrm{~mA})$ was carried out at $25^{\circ} \mathrm{C}$ with magnetic stirring until $2.1 F$ of electricity was consumed. The solution in the anodic chamber was collected and about half the amount of the solvent was removed under reduced pressure. The reaction mixture was analyzed by ESI-MS. HRMS (ESI) calcd for $\mathrm{C}_{27} \mathrm{H}_{26} \mathrm{NO}_{3} \mathrm{~S}_{2}\left(\mathrm{M}^{+}\right)$: 476.1349, found: 476.1359.

\section{Reactions of 3 with Benzofuran}

\section{Preparation of 1-Bromo-2-methoxy-4,5-dimethylbenzene $(1 \mathrm{e})^{4}$}

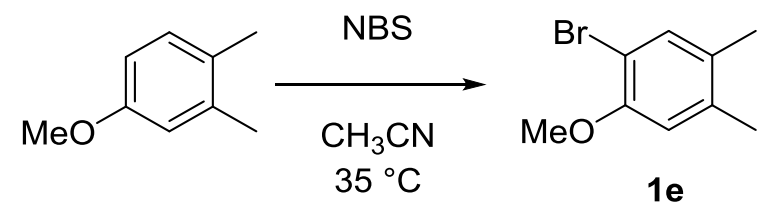

To a round-bottom flask were added 1,2-dimethylanisole $(1.4 \mathrm{~mL}, 10 \mathrm{mmol})$ and $\mathrm{CH}_{3} \mathrm{CN}(50 \mathrm{~mL})$. After addition of $\mathrm{N}$-bromosuccinimide $(1.95 \mathrm{~g}, 10.9 \mathrm{mmol})$, the mixture was stirred at $35^{\circ} \mathrm{C}$ for $20 \mathrm{~h}$. The solvent was removed under reduced pressure. The residue was dissolved in hexane and the precipitate was separated by filtration. The filtrate was concentrated in vacuum and the residue was purified by recrystallization from hexane to give 1-bromo-2-methoxy-4,5-dimethylbenzene (1e) in 45\% yield (968 mg, $4.5 \mathrm{mmol}$ ). TLC $\mathrm{R}_{f}$ 0.62 (hexane/EtOAc 5:1); ${ }^{1} \mathrm{H}$ NMR (400 MHz, $\mathrm{CDCl}_{3}$ ) $\delta 2.17$ (s, 3H), $2.22(\mathrm{~s}, 3 \mathrm{H}), 3.86(\mathrm{~s}, 3 \mathrm{H}), 6.69$ (s, $\left.1 \mathrm{H}\right)$, $7.28(\mathrm{~s}, 1 \mathrm{H}) ;{ }^{13} \mathrm{C}$ NMR $\left(100 \mathrm{MHz}, \mathrm{CDCl}_{3}\right) \delta 18.6,19.9,56.3,107.8,113.4,130.0,133.8,136.9,153.6$; HRMS (EI) calcd for $\mathrm{C}_{9} \mathrm{H}_{11} \mathrm{BrO}\left(\mathrm{M}^{+}\right)$: 213.9988, found: 213.9985 .

\section{Preparation of 2-(4-Methoxyphenyl)-1-phenylethanone (1g)}<smiles>COc1ccc(CC(=O)Cl)cc1</smiles>

To a round-bottom flask were added 4-methoxyphenylacetyl chloride $(4.6 \mathrm{~mL}, 30 \mathrm{mmol})$ and THF (20 
$\mathrm{mL})$. Phenylmagnesium bromide $(1.0 \mathrm{M}$ in THF, $30 \mathrm{~mL}, 30 \mathrm{mmol})$ was added dropwise at $-78{ }^{\circ} \mathrm{C}$. After being stirred at room temperature for $17 \mathrm{~h}$, the mixture was diluted with EtOAc, washed with $\mathrm{H}_{2} \mathrm{O}$, and dried over $\mathrm{Na}_{2} \mathrm{SO}_{4}$. After removal of the solvent under reduced pressure, the residue was purified by flash chromatography (hexane/EtOAc 5/1) and recrystallized from hexane/EtOAc to give 2-(4-methoxyphenyl)-1-phenylethanone (1g) in $16 \%$ yield $(1.1 \mathrm{~g}, 4.8 \mathrm{mmol})$. TLC $\mathrm{R}_{f} 0.33$ (hexane/EtOAc 5:1); ${ }^{1} \mathrm{H}$ NMR (400 MHz, $\left.\mathrm{CDCl}_{3}\right) \delta 3.78(\mathrm{~s}, 3 \mathrm{H}), 4.22(\mathrm{~s}, 2 \mathrm{H}), 6.86(\mathrm{~d}, J=8.8 \mathrm{~Hz}, 2 \mathrm{H}), 7.18(\mathrm{~d}, J=8.8 \mathrm{~Hz}$, $2 \mathrm{H}), 7.45(\mathrm{dd}, J=7.2,7.2 \mathrm{~Hz}, 2 \mathrm{H}), 7.55(\mathrm{tt}, J=1.6,7.2 \mathrm{~Hz}, 1 \mathrm{H}), 8.01(\mathrm{~d}, J=7.2 \mathrm{~Hz}, 2 \mathrm{H})$; The ${ }^{1} \mathrm{H}$ NMR spectrum is in agreement with that in the literature. ${ }^{6}$

Typical procedure for the generation of benzylaminosulfonium ions and their reactions with benzofuran

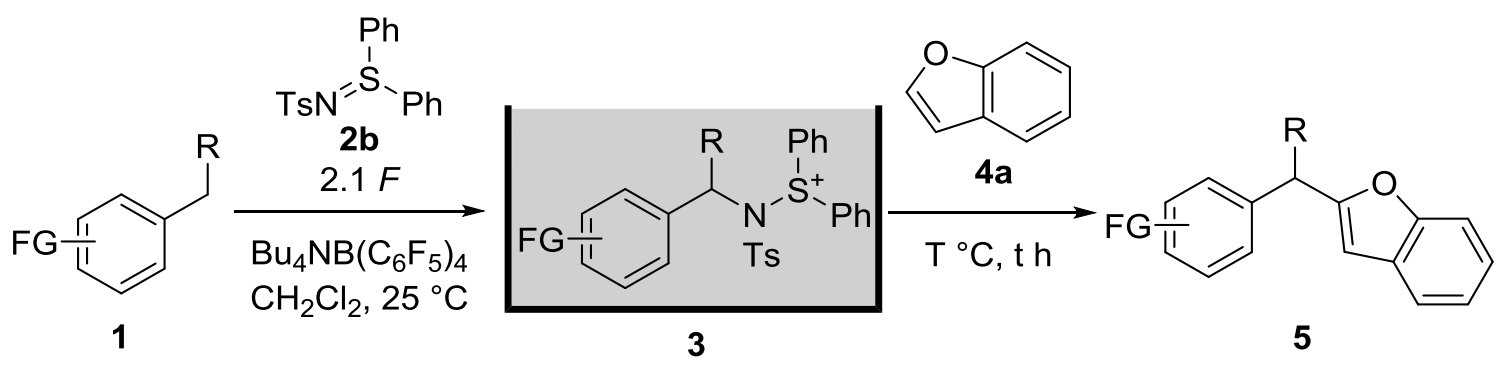

In the anodic chamber were placed benzylic substrate $\mathbf{1}(0.10 \mathrm{mmol})$, $S, S$-diphenyl- $N$-(4-methylbenzenesulfonyl)sulfilimine $\quad(\mathbf{2 b}) \quad(355 \quad \mathrm{mg}, \quad 1.00 \quad \mathrm{mmol}), \quad$ and $\quad 0.1 \quad \mathrm{M}$ $\mathrm{Bu}_{4} \mathrm{NB}\left(\mathrm{C}_{6} \mathrm{~F}_{5}\right)_{4} / \mathrm{CH}_{2} \mathrm{Cl}_{2}(10 \mathrm{~mL})$. In the cathodic chamber were placed TfOH $(30 \mu \mathrm{L}, 0.68 \mathrm{mmol})$ and $0.1 \mathrm{M}$ $\mathrm{Bu}_{4} \mathrm{NB}\left(\mathrm{C}_{6} \mathrm{~F}_{5}\right)_{4} / \mathrm{CH}_{2} \mathrm{Cl}(10 \mathrm{~mL})$. The constant current electrolysis $(4.0 \mathrm{~mA})$ was carried out at $25{ }^{\circ} \mathrm{C}$ with magnetic stirring until $2.1 F$ of electricity was consumed. Benzofuran $(\mathbf{4 a})(54 \mu \mathrm{L}, 0.50 \mathrm{mmol})$ was added to the anodic chamber, and the resulting mixture was stirred $\mathrm{T}{ }^{\circ} \mathrm{C}$ for $\mathrm{t} \mathrm{h}$ (Tables 2 and 3 ). The solution in the anodic chamber was collected and the solvent was removed under reduced pressure. The crude product was purified by flash chromatography and GPC to obtain the coupling product $\mathbf{5}$.

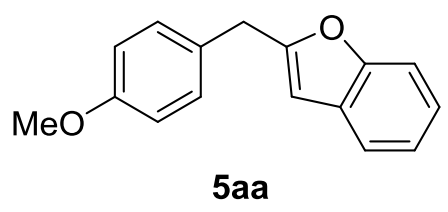

\section{2-(4-methoxybenzyl)benzofuran (5aa).}

Electrochemical oxidation (2.1 F) of 4-methoxytoluene (1a) $(12.5 \mathrm{mg}$, $0.102 \mathrm{mmol})$ in the presence of $\mathbf{2 b}(355 \mathrm{mg}, 1.00 \mathrm{mmol})$, and the treatment with benzofuran followed by flash chromatography (hexane/EtOAc 100:0 to 50:1 to $\mathrm{Et}_{2} \mathrm{O}$ ) and GPC gave the title compound (18.9 mg, 0.079 mmol, 77\%). TLC $\mathrm{R}_{f} 0.50$ (hexane/EtOAc 5:1); ${ }^{1} \mathrm{H}$ NMR $\left(400 \mathrm{MHz}, \mathrm{CDCl}_{3}\right) \delta 3.80(\mathrm{~s}, 3 \mathrm{H}), 4.05(\mathrm{~s}, 2 \mathrm{H}), 6.34(\mathrm{~s}, 1 \mathrm{H}), 6.87(\mathrm{~d}, J=$ 6.8 Hz, 2H), 7.15-7.24 (m, 4H), 7.38-7.42 (m, 1H), 7.44-7.48 (m, 1H); The ${ }^{1} \mathrm{H}$ NMR spectrum is in agreement with that in the literature. ${ }^{7}$

$S, S$-diphenyl- $N$-(4-methylbenzenesulfonyl)sulfilimine

(2b) was recovered in $100 \%$ yield $(354 \mathrm{mg}, 1.00 \mathrm{mmol}$ ) by flash chromatography followed by GPC. 
<smiles>COc1ccc(Cc2cc3ccccc3o2)cc1Br</smiles><smiles>COc1ccc(Cc2cc3ccccc3o2)cc1I</smiles><smiles>COC(=O)c1cc(Cc2cc3ccccc3o2)ccc1OC</smiles>

\section{2-(3-bromo-4-methoxybenzyl)benzofuran (5ba).}

Electrochemical oxidation $(2.1 F)$ of 3-bromo-4-methoxytoluene (1b) $\left(18.6 \mathrm{mg}, 0.092 \mathrm{mmol}\right.$ ) and the treatment with benzofuran ${ }^{8}$ followed by flash chromatography (hexane/EtOAc 100:0 to 50:1) gave the title compound (28.0 mg, $0.077 \mathrm{mmol}, 88 \%$ ). TLC $\mathrm{R}_{f} 0.45$ (hexane/EtOAc 5:1); ${ }^{1} \mathrm{H}$ NMR (400 MHz, $\left.\mathrm{CDCl}_{3}\right) \delta 3.87$ (s, 3H), 4.02 (s, 2H), 6.38 (s, $1 \mathrm{H}), 6.84(\mathrm{~d}, J=8.3 \mathrm{~Hz}, 1 \mathrm{H}), 7.14-7.24(\mathrm{~m}, 3 \mathrm{H}), 7.37-7.42(\mathrm{~m}, 1 \mathrm{H})$, 7.45-7.50 (m, 2H); ${ }^{13} \mathrm{C}$ NMR (100 MHz, $\left.\mathrm{CDCl}_{3}\right) \delta 33.7,56.3,103.4$, $110.9,111.7,112.0,120.4,122.6,123.5,128.7,128.8,130.8,133.6$, 154.8, 155.0, 157.3; HRMS (EI) calcd for $\mathrm{C}_{16} \mathrm{H}_{13} \mathrm{BrO}_{2}\left(\mathrm{M}^{+}\right)$: 316.0099, found: 316.0096 .

\section{2-(3-iodo-4-methoxybenzyl)benzofuran (5ca).}

Electrochemical oxidation $(2.1 F)$ of 3-iodo-4-methoxytoluene (1c) $\left(21.7 \mathrm{mg}, 0.088 \mathrm{mmol}\right.$ ) and the treatment with benzofuran ${ }^{8}$ followed by flash chromatography (hexane/EtOAc 100:0 to 50:1) gave the title compound (28.0 mg, $0.077 \mathrm{mmol}, 88 \%$ ). TLC $\mathrm{R}_{f} 0.44$ (hexane/EtOAc 5:1); ${ }^{1} \mathrm{H}$ NMR (400 MHz, $\mathrm{CDCl}_{3}$ ) $\delta 3.86(\mathrm{~s}, 3 \mathrm{H}), 4.00(\mathrm{~s}, 2 \mathrm{H}), 6.37$ (s, $1 \mathrm{H}), 6.77(\mathrm{~d}, J=8.4 \mathrm{~Hz}, 1 \mathrm{H}), 7.15-7.26(\mathrm{~m}, 3 \mathrm{H}), 7.38-7.42(\mathrm{~m}, 1 \mathrm{H})$, 7.46-7.50 (m, 1H), $7.72(\mathrm{~d}, J=2.2 \mathrm{~Hz}, 1 \mathrm{H}),{ }^{13} \mathrm{C} \mathrm{NMR}\left(100 \mathrm{MHz}, \mathrm{CDCl}_{3}\right)$ $\delta 33.5,56.4,86.0,103.4,110.87,110.92,120.4,122.6,123.5,128.7$, 129.9, 131.3, 139.7, 154.9, 157.0, 157.4; HRMS (ESI) calcd for $\mathrm{C}_{16} \mathrm{H}_{14} \mathrm{IO}_{2}\left(\mathrm{M}+\mathrm{H}^{+}\right)$: 365.0033 , found: 365.0030 .

\section{2-[4-methoxy-3-(methoxycarbonyl)benzyl]benzofuran (5da).}

Electrochemical oxidation $(2.1 \quad F)$ of 4-methoxy-3(methoxycarbonyl)toluene (1d) $(18.0 \mathrm{mg}, 0.100 \mathrm{mmol})$ and the treatment with benzofuran ${ }^{8}$ followed by flash chromatography (hexane/EtOAc 100:0 to 10:1) and GPC gave the title compound (23.1 mg, $0.078 \mathrm{mmol}$, 78\%). TLC $\mathrm{R}_{f} 0.19$ (hexane/EtOAc 5:1); ${ }^{1} \mathrm{H}$ NMR (400 MHz, $\mathrm{CDCl}_{3}$ ) $\delta$ 3.88 (s, $3 \mathrm{H}), 3.89$ (s, $3 \mathrm{H}), 4.06$ (s, $2 \mathrm{H}), 6.36$ (s, 1H), 6.94 (d, J = 8.8 Hz, 1H), 7.15-7.24 (m, 2H), 7.38-7.43 (m, 2H), 7.45-7.49 (m, 1H), 7.75 (d, $J$ $=2.2 \mathrm{~Hz}, 1 \mathrm{H}) ;{ }^{13} \mathrm{C} \mathrm{NMR}\left(100 \mathrm{MHz}, \mathrm{CDCl}_{3}\right) \delta 33.9,52.0,56.1,103.4$, $110.9,112.3,120.0,120.4,122.5,123.5,128.7,128.9,132.0,133.9$, 154.9, 157.5, 158.0, 166.5; HRMS (ESI) calcd for $\mathrm{C}_{18} \mathrm{H}_{17} \mathrm{O}_{4}\left(\mathrm{M}+\mathrm{H}^{+}\right)$: 297.1121, found: 297.1112 . 
<smiles>COc1cc(C)c(Cc2cc3ccccc3o2)cc1Br</smiles><smiles>COC(=O)C(c1ccc(OC)cc1)c1cc2ccccc2o1</smiles>

$5 f a$<smiles>COc1ccc(C(C(=O)c2ccccc2)c2cc3ccccc3o2)cc1</smiles>
$5 g a$

\section{2-[(5-bromo-4-methoxy-2-methylphenyl)methyl]benzofuran (5ea).}

Electrochemical oxidation ( $2.1 F$ ) of 5-bromo-4-methoxy-2-

methyltoluene (1e) $(21.5 \mathrm{mg}, 0.100 \mathrm{mmol})$ and the treatment with benzofuran $^{8}$ followed by flash chromatography (hexane/EtOAc 100:0 to 20:1) and GPC gave the title compound (24.4 mg, $0.074 \mathrm{mmol}, 74 \%$ ). TLC $\mathrm{R}_{f} 0.55$ (hexane/EtOAc 5:1); ${ }^{1} \mathrm{H}$ NMR (400 MHz, $\mathrm{CDCl}_{3}$ ) $\delta 2.31$ (s, $3 \mathrm{H}), 3.88(\mathrm{~s}, 3 \mathrm{H}), 4.00(\mathrm{~s}, 2 \mathrm{H}), 6.24(\mathrm{~s}, 1 \mathrm{H}), 6.74(\mathrm{~s}, 1 \mathrm{H}), 7.14-7.24(\mathrm{~m}$, 2H), 7.38-7.47 (m, 3H); ${ }^{13} \mathrm{C}$ NMR (100 MHz, $\left.\mathrm{CDCl}_{3}\right) \delta$ 19.6, 31.7, 56.2, 103.3, 108.4, 110.9, 114.0, 120.4, 122.5, 123.4, 128.7, 128.9, 134.2, 137.2, 154.6, 154.8, 157.0; HRMS (EI) calcd for $\mathrm{C}_{16} \mathrm{H}_{13} \mathrm{BrO}_{2}\left(\mathrm{M}^{+}\right)$: 330.0250, found: 330.0256 .

\section{2-[(methoxycarbonyl)-(4-methoxyphenyl)methyl]benzofuran (5fa). \\ Electrochemical oxidation $(2.1 F)$ of (methoxycarbonyl)-} (4-methoxyphenyl)methane (1f) $(17.9 \mathrm{mg}, 0.099 \mathrm{mmol})$ and the treatment with benzofuran ${ }^{8}$ followed by flash chromatography (hexane/EtOAc 100:0 to 20:1) and GPC gave the title compound (22.4 $\mathrm{mg}, 0.074 \mathrm{mmol}, 76 \%$ ). TLC $\mathrm{R}_{f} 0.45$ (hexane/EtOAc 5:1); ${ }^{1} \mathrm{H}$ NMR (400 $\left.\mathrm{MHz}, \mathrm{CDCl}_{3}\right) \delta 3.76(\mathrm{~s}, 3 \mathrm{H}), 3.80(\mathrm{~s}, 3 \mathrm{H}), 5.09(\mathrm{~s}, 1 \mathrm{H}), 6.56(\mathrm{~s}, 1 \mathrm{H}), 6.90$ $(\mathrm{d}, J=8.8 \mathrm{~Hz}, 2 \mathrm{H}), 7.16-7.26(\mathrm{~m}, 2 \mathrm{H}), 7.36(\mathrm{~d}, J=8.8 \mathrm{~Hz}, 2 \mathrm{H})$, 7.40-7.44 (m, 1H), 7.47-7.51 (m, 1H); ${ }^{13} \mathrm{C}$ NMR (100 MHz, $\left.\mathrm{CDCl}_{3}\right) \delta$ 50.8, 52.7, 55.3, 105.0, 111.1, 114.2, 120.9, 122.7, 124.0, 127.4, 128.2, 129.7, 154.95, 154.97, 159.4, 170.8; HRMS (ESI) calcd for $\mathrm{C}_{18} \mathrm{H}_{16} \mathrm{O}_{4} \mathrm{Na}$ $\left(\mathrm{M}+\mathrm{Na}^{+}\right): 319.0941$, found: 319.0940 .

\section{2-[(4-methoxyphenyl)-(phenylcarbonyl)methyl]benzofuran (5ga).}

Electrochemical oxidation $\quad(2.1 \quad F) \quad$ of (4-methoxyphenyl)(phenylcarbonyl)methane (1g) (22.3 mg, $0.099 \mathrm{mmol})$ and the treatment with benzofuran ${ }^{8}$ followed by flash chromatography (hexane/EtOAc 100:0 to 50:1 to 20:1) and GPC gave the title compound (23.2 mg, $0.068 \mathrm{mmol}, 68 \%$ ). TLC $\mathrm{R}_{f} 0.38$ (hexane/EtOAc 5:1); ${ }^{1} \mathrm{H}$ NMR $\left(400 \mathrm{MHz}, \mathrm{CDCl}_{3}\right) \delta 3.78(\mathrm{~s}, 3 \mathrm{H}), 6.09(\mathrm{~s}, 1 \mathrm{H}), 6.47(\mathrm{~s}, 1 \mathrm{H}), 6.90(\mathrm{~d}, J=$ $8.8 \mathrm{~Hz}, 2 \mathrm{H}), 7.14-7.26(\mathrm{~m}, 2 \mathrm{H}), 7.35(\mathrm{~d}, J=8.8 \mathrm{~Hz}, 2 \mathrm{H}), 7.38-7.48(\mathrm{~m}$, $4 \mathrm{H}), 7.53(\mathrm{t}, J=7.5 \mathrm{~Hz}, 1 \mathrm{H}) 8.03(\mathrm{~d}, J=7.0 \mathrm{~Hz}, 2 \mathrm{H}) ;{ }^{13} \mathrm{C}$ NMR $(100$ $\left.\mathrm{MHz}, \mathrm{CDCl}_{3}\right) \delta 52.9,55.2,105.9,111.1,114.4,120.8,122.6,123.9$, $127.6,128.4,128.7,128.9,130.2,133.3,136.1,155.0,156.1,159.3$, 195.4; HRMS (ESI) calcd for $\mathrm{C}_{23} \mathrm{H}_{19} \mathrm{O}_{3}\left(\mathrm{M}+\mathrm{H}^{+}\right)$: 343.1329, found: 343.1325 . 

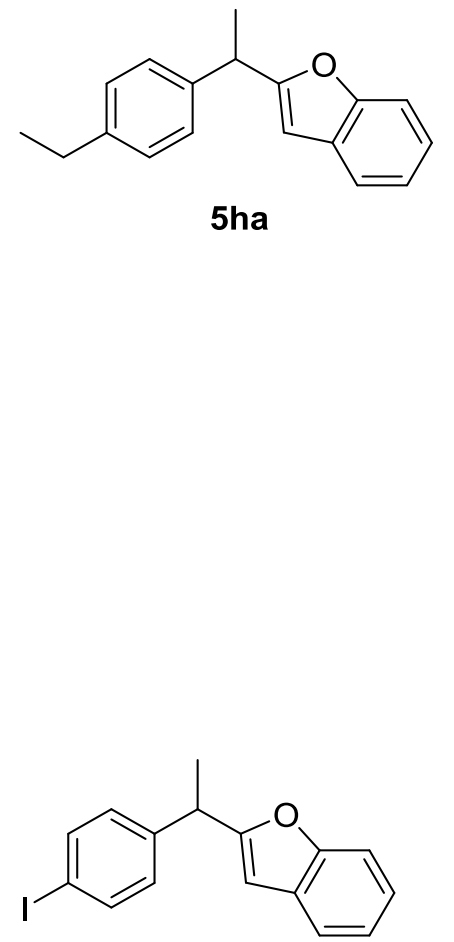

5 ia

\section{2-[1-(4-ethylphenyl)ethyl]benzofuran (5ha).}

Electrochemical oxidation $(3.0 \mathrm{~F})$ of $p$-diethylbenzene $(\mathbf{1 h})(13.1 \mathrm{mg}$, $0.098 \mathrm{mmol})$ at $0{ }^{\circ} \mathrm{C}$ and the treatment with benzofuran ${ }^{8}$ followed by flash chromatography (hexane/EtOAc 100:0 to 100:1) and GPC gave the title compound (15.6 mg, $0.062 \mathrm{mmol}, 64 \%$ ). TLC $\mathrm{R}_{f} 0.59$ (hexane/EtOAc 5:1); ${ }^{1} \mathrm{H}$ NMR (400 MHz, $\mathrm{CDCl}_{3}$ ) $\delta 1.22$ (t, $\left.J=7.4 \mathrm{~Hz}, 3 \mathrm{H}\right), 1.68$ (d, $J=$ $7.0 \mathrm{~Hz}, 3 \mathrm{H}$ ), 2.63 (q, $J=7.4 \mathrm{~Hz}, 2 \mathrm{H}), 4.22$ (q, $J=7.5 \mathrm{~Hz}, 1 \mathrm{H}), 6.42$ (s, $1 \mathrm{H}), 7.12-7.22(\mathrm{~m}, 6 \mathrm{H}), 7.36-7.40(\mathrm{~m}, 1 \mathrm{H}), 7.46-7.50(\mathrm{~m}, 1 \mathrm{H}) ;{ }^{13} \mathrm{C}$ NMR (100 MHz, $\left.\mathrm{CDCl}_{3}\right) \delta 15.5,20.4,28.4,39.2,101.9,110.9,120.4$, $122.4,123.3,127.4,128.0,128.6,140.5,142.6,154.8,162.4$; HRMS (APCI) calcd for $\mathrm{C}_{18} \mathrm{H}_{19} \mathrm{O}\left(\mathrm{M}+\mathrm{H}^{+}\right)$: 251.1430, found: 251.1421 .

\section{2-[1-(4-iodophenyl)ethyl]benzofuran (5ia).}

Electrochemical oxidation (2.5 F) of 1-ethyl-4-iodobenzene (1i) (46.1 $\mathrm{mg}, 0.199 \mathrm{mmol})$ and the treatment with benzofuran ${ }^{8}$ followed by flash chromatography (hexane/EtOAc 100:0 to 50:1) and GPC gave the title compound (42.3 mg, $0.121 \mathrm{mmol}, 61 \%$ ). TLC $\mathrm{R}_{f} 0.21$ (hexane/EtOAc 50:1); ${ }^{1} \mathrm{H}$ NMR (400 MHz, $\left.\mathrm{CDCl}_{3}\right) \delta 1.66(\mathrm{~d}, J=7.4 \mathrm{~Hz}, 3 \mathrm{H}), 4.19$ (q, $J=$ $7.0 \mathrm{~Hz}, 1 \mathrm{H}), 6.43(\mathrm{~s}, 1 \mathrm{H}), 7.03(\mathrm{~d}, J=8.4 \mathrm{~Hz}, 2 \mathrm{H}), 7.15-7.23(\mathrm{~m}, 2 \mathrm{H})$, 7.36-7.39 (m, 1H), 7.47-7.51 (m, 1H), $7.63(\mathrm{~d}, J=8.4 \mathrm{~Hz}, 2 \mathrm{H}) ;{ }^{13} \mathrm{C}$ NMR (100 MHz, $\left.\mathrm{CDCl}_{3}\right) \delta 20.1,39.2,92.0,102.3,111.0,120.5,122.5$, 123.6, 128.4, 129.5, 137.6, 143.0, 154.8, 161.2; HRMS (EI) calcd for $\mathrm{C}_{16} \mathrm{H}_{13} \mathrm{IO}\left(\mathrm{M}^{+}\right)$: 348.0006, found: 348.0007 .

\section{2-diphenylmethylbenzofuran (5ja).}

Electrochemical oxidation $(2.1 F)$ of diphenylmethane $(\mathbf{1 j})(15.7 \mathrm{mg}$, $0.093 \mathrm{mmol}$ ) and the treatment with benzofuran followed by flash chromatography (hexane/EtOAc 100:0 to 50:1) and GPC gave the title compound (23.8 mg, $0.059 \mathrm{mmol}, 63 \%$ ). TLC $\mathrm{R}_{f} 0.70$ (hexane/EtOAc 5:1); ${ }^{1} \mathrm{H}$ NMR (400 MHz, $\left.\mathrm{CDCl}_{3}\right) \delta 5.58(\mathrm{~s}, 1 \mathrm{H}), 6.27$ (s, 1H), 7.15-7.35 (m, 12H), 7.39-7.42 (m, 1H), 7.45-7.47 (m, 1H). The ${ }^{1} \mathrm{H}$ NMR spectrum is in agreement with that in the literature. ${ }^{9}$

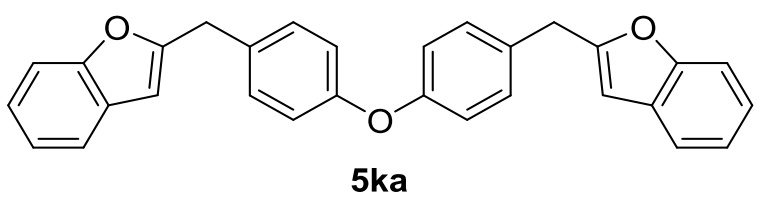

5 ka

\section{Bis[4-(2-benzofurylmethyl)phenyl]ether (5ka).}

Electrochemical oxidation $(4.1 F)$ of di-p-tolylether (1k) $(20.7 \mathrm{mg}, 0.104 \mathrm{mmol})$ and the treatment with benzofuran $^{8}$ followed by flash chromatography (hexane/EtOAc 100:0 to 50:1) and GPC gave the title 
compound (23.0 mg, $0.053 \mathrm{mmol}, 51 \%)$. TLC $\mathrm{R}_{f} 0.56$ (hexane/EtOAc 5:1); ${ }^{1} \mathrm{H}$ NMR (400 MHz, $\mathrm{CDCl}_{3}$ ) $\delta$ $4.08(\mathrm{~s}, 4 \mathrm{H}), 6.38(\mathrm{~s}, 2 \mathrm{H}), 6.96(\mathrm{~d}, J=8.3 \mathrm{~Hz}, 4 \mathrm{H})$, 7.15-7.28 (m, 8H), 7.39-7.43 (m, 2H), 7.46-7.49 (m, $2 \mathrm{H}) ;{ }^{13} \mathrm{C} \mathrm{NMR}\left(100 \mathrm{MHz}, \mathrm{CDCl}_{3}\right) \delta 34.2,103.3,110.9$, $118.9,120.4,122.5,123.4,128.7,130.2,132.0,154.9$, 156.0, 157.8; HRMS (EI) calcd for $\mathrm{C}_{30} \mathrm{H}_{22} \mathrm{O}_{3}\left(\mathrm{M}^{+}\right)$: 430.1563 , found: 430.1561 . 


\section{Oxidation Potential}

Rotating-disk electrode (RDE) voltammetry was carried out using BAS 600C and BAS RRDE-3 rotating disk electrodes. Measurements were carried out in $0.1 \mathrm{M} \mathrm{LiClO}_{4} / \mathrm{CH}_{3} \mathrm{CN}$ using a glassy carbon disk working electrode, a platinum wire counter electrode, and an SCE reference electrode with sweep rate of $10 \mathrm{mVs}^{-1}$ at $3000 \mathrm{rpm}$ at room temperatue. The substrate concentration was $10.0 \mathrm{mM}$.

Table S1. Oxidation Potentials of Toluene Derivatives, Sulfilimine 2b, and Benzofuran<smiles>COc1ccc(C)cc1</smiles>

$1 \mathrm{~b}$<smiles>COc1ccc(C)cc1I</smiles><smiles>COC(=O)c1cc(C)ccc1OC</smiles><smiles>COc1cc(C)c(C)cc1Br</smiles>

1e<smiles>COC(=O)Cc1ccc(OC)cc1</smiles>

$1 \mathrm{f}$<smiles>COc1ccc(CC(=O)c2ccccc2)cc1</smiles>

$1 \mathrm{~g}$
$1.38^{10}$<smiles>CCc1ccc(CC)cc1</smiles>

1h

1.59<smiles>CCc1ccc(I)cc1</smiles>

1.52

1.61<smiles>c1ccc(Cc2ccccc2)cc1</smiles>

1j<smiles>Cc1ccc(O)cc1</smiles>

$1 \mathrm{k}$

1.54<smiles>[As]N=S(c1ccccc1)c1ccccc1</smiles>

$2 b$<smiles>c1ccc2occc2c1</smiles>

\subsection{7}




\section{Reactions of 3a with Nucleophiles}

Typical procedure for the generation of benzylaminosulfonium ions and their reactions with nucleophiles

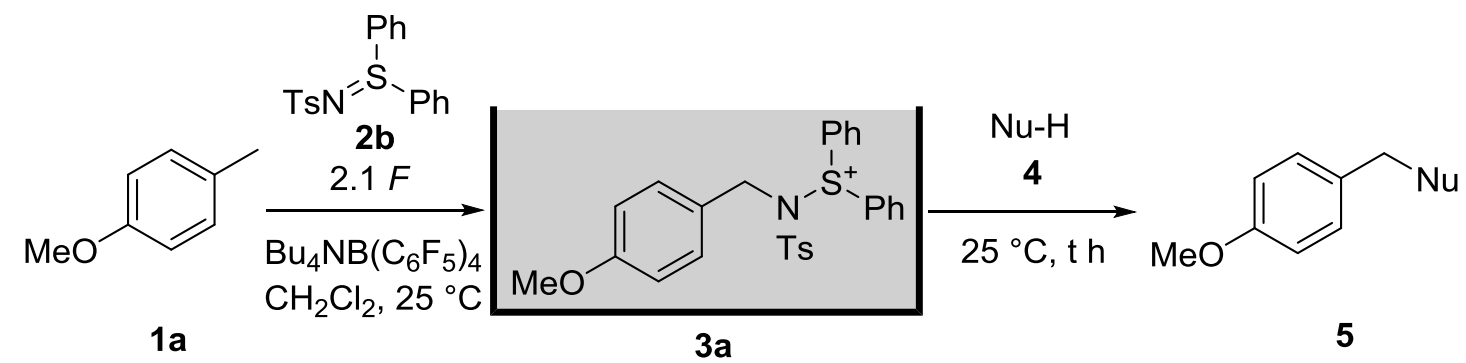

In the anodic chamber were placed 4-methoxytoluene (1) (12.2 $\mathrm{mg}, 0.10 \mathrm{mmol})$, $S, S$-diphenyl- $N$-(4-methylbenzenesulfonyl)sulfilimine $\quad(\mathbf{2 b}) \quad(355 \quad \mathrm{mg}, \quad 1.00 \quad \mathrm{mmol}), \quad$ and $\quad 0.1 \quad \mathrm{M}$ $\mathrm{Bu}_{4} \mathrm{NB}\left(\mathrm{C}_{6} \mathrm{~F}_{5}\right)_{4} / \mathrm{CH}_{2} \mathrm{Cl}_{2}(10 \mathrm{~mL})$. In the cathodic chamber were placed $\mathrm{TfOH}(30 \mu \mathrm{L}, 0.68 \mathrm{mmol})$ and $0.1 \mathrm{M}$ $\mathrm{Bu}_{4} \mathrm{NB}\left(\mathrm{C}_{6} \mathrm{~F}_{5}\right)_{4} / \mathrm{CH}_{2} \mathrm{Cl}(10 \mathrm{~mL})$. The constant current electrolysis $(4.0 \mathrm{~mA})$ was carried out at $25{ }^{\circ} \mathrm{C}$ with magnetic stirring until $2.1 F$ of electricity was consumed. Nucleophile $4(0.50 \mathrm{mmol})$ was added to the anodic chamber, and the resulting mixture was stirred $\mathrm{T}{ }^{\circ} \mathrm{C}$ for $\mathrm{t} \mathrm{h}$. The solution in the anodic chamber was collected and the solvent was removed under reduced pressure. The crude product was purified by flash chromatography and GPC to obtain the coupling product 5.<smiles>COc1ccc(Cc2c[nH]c3ccccc23)cc1</smiles>

$5 a b$<smiles>COc1ccc(Cc2csc3ccccc23)cc1</smiles>

\section{3-(4-methoxybenzyl)indole (5ab).}

Electrochemical oxidation $(2.1 F)$ of 4-methoxytoluene $(11.7 \mathrm{mg}$, $0.096 \mathrm{mmol}$ ) and the treatment with indole (4b) followed by flash chromatography (hexane/EtOAc 100:0 to 5:1) and GPC gave the title compound (17.1 mg, $0.072 \mathrm{mmol}, 75 \%$ ). TLC $\mathrm{R}_{f} 0.27$ (hexane/EtOAc 5:1); ${ }^{1} \mathrm{H}$ NMR (400 MHz, $\left.\mathrm{CDCl}_{3}\right) \delta 3.78(\mathrm{~s}, 3 \mathrm{H}), 4.06(\mathrm{~s}, 2 \mathrm{H}), 6.82(\mathrm{~d}, J=$ $8.8 \mathrm{~Hz}, 2 \mathrm{H}), 6.88-6.91(\mathrm{~m}, 1 \mathrm{H}), 7.07$ (ddd, $J=1.2,7.6,8.4 \mathrm{~Hz}, 1 \mathrm{H})$, 7.15-7.22 (m, 3H), 7.35 (td, $J=0.8,8.0 \mathrm{~Hz}, 1 \mathrm{H}), 7.49-7.53(\mathrm{~m}, 1 \mathrm{H}), 7.93$ (br, 1H). The ${ }^{1} \mathrm{H}$ NMR spectrum is in agreement with that in the literature. ${ }^{12}$

\section{3-(4-methoxybenzyl)benzo[b]thiophene (5ac).}

Electrochemical oxidation $(2.1 F)$ of 4-methoxytoluene $(11.8 \mathrm{mg}$, $0.097 \mathrm{mmol}$ ) and the treatment with benzo[b]thiophene (4c) followed by flash chromatography (hexane/EtOAc 100:0 to 50:1) and GPC gave the title compound (13.9 mg, $0.054 \mathrm{mmol}, 56 \%$ ). TLC $\mathrm{R}_{f} 0.60$ (hexane/EtOAc 5:1); ${ }^{1} \mathrm{H}$ NMR (400 MHz, $\left.\mathrm{CDCl}_{3}\right) \delta 3.79$ (s, 3H), 4.13 (s, 2H), 6.84 (d, $J=$ $8.4 \mathrm{~Hz}, 2 \mathrm{H}), 6.98(\mathrm{~s}, 1 \mathrm{H}), 7.18(\mathrm{~d}, J=8.8 \mathrm{~Hz}, 2 \mathrm{H}), 7.32-7.35(\mathrm{~m}, 2 \mathrm{H})$, 7.68-7.71 $(\mathrm{m}, 1 \mathrm{H}), 7.84-7.87(\mathrm{~m}, 1 \mathrm{H})$. The ${ }^{1} \mathrm{H}$ NMR spectrum is in 
agreement with that in the literature. ${ }^{13}$<smiles>COc1ccc(Cc2ccc(OC)cc2)cc1</smiles><smiles>COc1ccc(Cc2ccc(O)cc2)cc1</smiles>
5 ae<smiles>COc1ccc(Cc2cc(C)ccc2O)cc1</smiles>

5af

\section{di(4-methoxyphenyl)methane (5ad).}

Electrochemical oxidation $(2.1 \mathrm{~F})$ of 4-methoxytoluene $(11.6 \mathrm{mg}$, $0.095 \mathrm{mmol}$ ) and the treatment with anisole (4d) followed by flash chromatography (hexane/EtOAc 100:0 to 20:1) and GPC gave the title compound (14.2 mg, $0.062 \mathrm{mmol}, 65 \%$ ). TLC $\mathrm{R}_{f} 0.57$ (hexane/EtOAc 5:1); ${ }^{1} \mathrm{H}$ NMR (400 MHz, $\left.\mathrm{CDCl}_{3}\right) \delta 3.78(\mathrm{~s}, 6 \mathrm{H}), 3.86(\mathrm{~s}, 4 \mathrm{H}), 6.82(\mathrm{~d}, J=$ $8.4 \mathrm{~Hz}, 4 \mathrm{H}), 7.09(\mathrm{~d}, J=8.4 \mathrm{~Hz}, 4 \mathrm{H})$. The ${ }^{1} \mathrm{H}$ NMR spectrum is in agreement with that in the literature. ${ }^{14}$

\section{4-(4-methoxybenzyl)phenol (5ae).}

Electrochemical oxidation $(2.1 F)$ of 4-methoxytoluene $(11.0 \mathrm{mg}$, $0.090 \mathrm{mmol}$ ) and the treatment with phenol (4e) followed by flash chromatography (hexane/EtOAc 100:0 to 5:1) gave the title compound (14.8 mg, $0.069 \mathrm{mmol}, 77 \%$ ). TLC $\mathrm{R}_{f} 0.60$ (hexane/EtOAc 2:1); ${ }^{1} \mathrm{H}$ NMR $\left(400 \mathrm{MHz}, \mathrm{CDCl}_{3}\right) \delta 3.78$ (s, 3H), 3.85 (s, 2H), 4.82 (br, 1H), 6.74 (d, $J=$ $8.8 \mathrm{~Hz}, 2 \mathrm{H}), 6.82(\mathrm{~d}, J=8.8 \mathrm{~Hz}, 2 \mathrm{H}), 7.03(\mathrm{~d}, J=8.4 \mathrm{~Hz}, 2 \mathrm{H}), 7.08$ (d, $J=$ $8.0 \mathrm{~Hz}, 2 \mathrm{H})$. The ${ }^{1} \mathrm{H}$ NMR spectrum is in agreement with that in the literature. $^{15,16}$

\section{2-(4-methoxybenzyl)-4-methylphenol (5af).}

Electrochemical oxidation $(2.1 F)$ of 4-methoxytoluene $(11.5 \mathrm{mg}$, $0.094 \mathrm{mmol}$ ) and the treatment with $p$-cresol (4f) followed by flash chromatography (hexane/EtOAc 100:0 to 10:1) and GPC gave the title compound (18.0 mg, $0.079 \mathrm{mmol}, 84 \%$ ). TLC R 0.27 (hexane/EtOAc 5:1); ${ }^{1} \mathrm{H}$ NMR (400 MHz, $\left.\mathrm{CDCl}_{3}\right) \delta 2.25$ (s, 3H), 3.78 (s, 3H), 3.89 (s, 2H), 4.56 (br, 1H), $6.68(\mathrm{~d}, J=8.3 \mathrm{~Hz}, 1 \mathrm{H}), 6.83(\mathrm{~d}, J=8.8 \mathrm{~Hz}, 2 \mathrm{H})$, 6.84-6.92 (m, 2H), $7.14(\mathrm{~d}, J=8.4 \mathrm{~Hz}, 2 \mathrm{H}) ;{ }^{13} \mathrm{C}$ NMR (100 MHz, $\left.\mathrm{CDCl}_{3}\right)$ $\delta$ 20.5, 35.6, 55.2, 114.1, 115.6, 126.9, 128.1, 129.6, 130.1, 131.4, 131.8, 151.5, 158.1; HRMS (EI) calcd for $\mathrm{C}_{15} \mathrm{H}_{16} \mathrm{O}_{2}\left(\mathrm{M}^{+}\right)$: 228.1150, found: 228.1150

\section{1-methoxy -4-(4-methoxybenzyl)naphthalene (5ag).}

Electrochemical oxidation $(2.1 F)$ of 4-methoxytoluene $(11.6 \mathrm{mg}$, $0.095 \mathrm{mmol})$ and the treatment with 1-methoxynaphthalene $(\mathbf{4 g})$ followed by flash chromatography (hexane/EtOAc 100:0 to 50:1) and GPC gave the title compound (21.8 mg, $0.078 \mathrm{mmol}, 82 \%$ ). TLC $\mathrm{R}_{f} 0.47$ (hexane/EtOAc 
<smiles>COc1ccc(CC(C(C)=O)C(C)=O)cc1</smiles><smiles>COc1ccc(C/C(C(C)=O)=C(\C)O)cc1</smiles><smiles>COC(=O)C(C)(C)Cc1ccc(OC)cc1</smiles>

5ai<smiles>C=CCCc1ccc(OC)cc1</smiles>

5aj
5:1); ${ }^{1} \mathrm{H}$ NMR (400 MHz, $\left.\mathrm{CDCl}_{3}\right) \delta 3.75(\mathrm{~s}, 3 \mathrm{H}), 3.98(\mathrm{~s}, 3 \mathrm{H}), 4.30(\mathrm{~s}$, 2H), $6.74(\mathrm{~d}, J=7.6 \mathrm{~Hz}, 1 \mathrm{H}), 6.79(\mathrm{~d}, J=8.4 \mathrm{~Hz}, 2 \mathrm{H}), 7.09(\mathrm{~d}, J=8.0 \mathrm{~Hz}$, $2 \mathrm{H}), 7.16(\mathrm{~d}, J=8.0 \mathrm{~Hz}, 1 \mathrm{H}), 7.42-7.47(\mathrm{~m}, 2 \mathrm{H}), 7.87-7.94(\mathrm{~m}, 1 \mathrm{H})$, $8.26-8.32(\mathrm{~m}, 1 \mathrm{H})$. The ${ }^{1} \mathrm{H}$ NMR spectrum is in agreement with that in the literature. ${ }^{17}$

\section{3-(4-methoxybenzyl)-2,4-pentanedione (5ah).}

Electrochemical oxidation $(2.1 F)$ of 4-methoxytoluene $(11.5 \mathrm{mg}$, $0.094 \mathbf{m m o l}$ ) and the treatment with 2,4-pentanedione (4h) followed by flash chromatography (hexane/EtOAc 100:0 to 50:1 to 5:1) gave the title compound (18.3 mg, $0.083 \mathrm{mmol}, 88 \%$, 2:3 mixture of 5ah and 5ah'). TLC $\mathrm{R}_{f} 0.26$ (hexane/EtOAc 5:1); ${ }^{1} \mathrm{H}$ NMR (400 MHz, $\mathrm{CDCl}_{3}$ ) 5ah $\delta 2.08$ (s, 6H), 3.09 (d, $J=7.4 \mathrm{~Hz}, 2 \mathrm{H}), 3.78(\mathrm{~s}, 3 \mathrm{H}), 3.97(\mathrm{t}, J=7.5 \mathrm{~Hz}, 1 \mathrm{H})$ 6.80-6.87 (m, 2H), 7.04-7.10 (m, 2H); 5ah $\delta 2.12(\mathrm{~s}, 6 \mathrm{H}), 3.59(\mathrm{~s}, 2 \mathrm{H})$, $3.79(\mathrm{~s}, 3 \mathrm{H}), 6.80-6.87(\mathrm{~m}, 2 \mathrm{H}), 7.04-7.10(\mathrm{~m}, 2 \mathrm{H}) ;{ }^{13} \mathrm{C} \mathrm{NMR}(100 \mathrm{MHz}$, $\left.\mathrm{CDCl}_{3}\right) \delta 23.2,29.7,32.0,33.5,55.2,55.3,70.2,108.6,114.0,114.1$, 128.3, 129.6, 129.9, 131.5, 158.1, 158.4, 191.9, 203.7; HRMS (EI) calcd for $\mathrm{C}_{13} \mathrm{H}_{16} \mathrm{O}_{3}\left(\mathrm{M}+\mathrm{Na}^{+}\right)$: 243.0992, found: 243.0991 .

methyl 3-(4-methoxyphenyl)-2,2-dimethylpropanoate (5ai).

Electrochemical oxidation $(2.1 F)$ of 4-methoxytoluene $(11.8 \mathrm{mg}$, $0.097 \mathrm{mmol}$ ) and the treatment with methyl trimethylsilyl dimethylketene acetal (4i) followed by flash chromatography (hexane/EtOAc 100:0 to $50: 1$ to $20: 1)$ and GPC gave the title compound $(14.4 \mathrm{mg}, 0.065 \mathrm{mmol}$, 67\%). TLC $\mathrm{R}_{f} 0.42$ (hexane/EtOAc 5:1); ${ }^{1} \mathrm{H}$ NMR (400 MHz, $\mathrm{CDCl}_{3}$ ) $\delta$ $1.16(\mathrm{~s}, 6 \mathrm{H}), 2.79$ (s, 2H), 3.65 (s, 3H), 3.78 (s, 3H), 6.80 (d, $J=8.8 \mathrm{~Hz}$, $2 \mathrm{H}), 7.01(\mathrm{~d}, J=8.4 \mathrm{~Hz}, 2 \mathrm{H})$. The ${ }^{1} \mathrm{H}$ NMR spectrum is in agreement with that in the literature. ${ }^{18}$

\section{4-(4-methoxyphenyl)-1-butene (5aj).}

Electrochemical oxidation $(2.1 \mathrm{~F})$ of 4-methoxytoluene $(11.4 \mathrm{mg}$, $0.093 \mathbf{m m o l})$ and the treatment with allyltrimethylsilane $(\mathbf{4 j}){ }^{19}$ followed by flash chromatography (hexane/EtOAc 100:0 to 50:1) gave the title compound (12.2 mg, $0.075 \mathrm{mmol}, 81 \%$ ). TLC $\mathrm{R}_{f} 0.64$ (hexane/EtOAc 5:1); ${ }^{1} \mathrm{H}$ NMR (400 MHz, $\left.\mathrm{CDCl}_{3}\right) \delta 2.30-2.38(\mathrm{~m}, 2 \mathrm{H}), 2.65(\mathrm{t}, J=7.2 \mathrm{~Hz}$, 2H), 3.79 (s, 3H), 4.90-5.07 (m, 2H), $6.80(\mathrm{tdd}, J=7.6,12.8,16.8 \mathrm{~Hz}$, $1 \mathrm{H}), 6.83(\mathrm{~d}, J=8.8 \mathrm{~Hz}, 2 \mathrm{H}), 7.10(\mathrm{~d}, J=8.0 \mathrm{~Hz}, 2 \mathrm{H})$; The ${ }^{1} \mathrm{H}$ NMR spectrum is in agreement with that in the literature. ${ }^{20}$ 
<smiles>COc1ccc(CC#Cc2ccccc2)cc1</smiles>

\section{3-(4-methoxyphenyl)-1-phenylpropyne (5ak).}

Electrochemical oxidation $(2.1 F)$ of 4-methoxytoluene $(10.7 \mathrm{mg}$, $0.088 \mathrm{mmol}$ ) and the treatment with 1-phenyl-2-(trimethylsilyl)acetylene (4k) followed by flash chromatography (hexane/EtOAc 100:0 to 50:1) and GPC gave the title compound (10.7 mg, $0.048 \mathrm{mmol}, 55 \%)$. TLC $\mathrm{R}_{f} 0.45$ (hexane/EtOAc 5:1); ${ }^{1} \mathrm{H}$ NMR (400 MHz, $\mathrm{CDCl}_{3}$ ) $\delta 3.77$ (s, 2H), 3.81 (s, $3 \mathrm{H}), 6.88(\mathrm{~d}, J=8.4 \mathrm{~Hz}, 2 \mathrm{H}), 7.27-7.30(\mathrm{~m}, 2 \mathrm{H}), 7.33(\mathrm{~d}, J=8.8 \mathrm{~Hz}, 2 \mathrm{H})$, $7.42-7.46(\mathrm{~m}, 2 \mathrm{H})$. The ${ }^{1} \mathrm{H}$ NMR spectrum is in agreement with that in the literature. ${ }^{21}$

\section{Synthesis of an Inhibitor of PTPase}

\section{Preparation of 2-Benzylbenzo $[b]$ thiophene $6^{22}$}
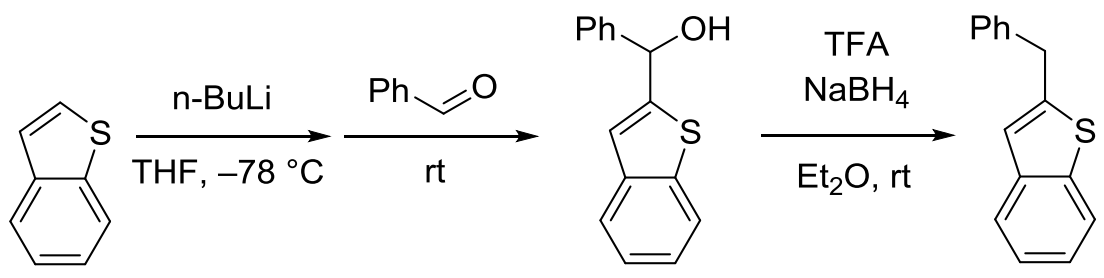

6

To a round-bottom flask were added benzo[b]thiophene $(3.72 \mathrm{~g}, 27.9 \mathrm{mmol})$ and THF $(100 \mathrm{~mL}) . n$-BuLi (2.5 $\mathrm{M}$ in hexane, $12 \mathrm{~mL}, 30 \mathrm{mmol}$ ) was added dropwise at $-78{ }^{\circ} \mathrm{C}$. After being stirred at $-78{ }^{\circ} \mathrm{C}$ for $1 \mathrm{~h}$, benzaldehyde $(3.2 \mathrm{~mL}, 32 \mathrm{mmol})$ was added to the mixture. After stirring at room temperature for $30 \mathrm{~min}$, the mixture was diluted with $\mathrm{Et}_{2} \mathrm{O}$, washed with sat. $\mathrm{NH}_{4} \mathrm{Cl}, \mathrm{H}_{2} \mathrm{O}$ and brine, and dried over $\mathrm{Na}_{2} \mathrm{SO}_{4}$. After removal of the solvent under reduced pressure, the residue was purified by recrystallization from hexane/ $\mathrm{Et}_{2} \mathrm{O}$ to give benzo[b]thiophen-2-yl-phenylmethanol in 74\% yield (4.96 g, $\left.20.6 \mathrm{mmol}\right)$. TLC $\mathrm{R}_{f}$ 0.21 (hexane/EtOAc 5:1); ${ }^{1} \mathrm{H}$ NMR $\left(400 \mathrm{MHz}, \mathrm{CDCl}_{3}\right) \delta 2.49(\mathrm{~d}, J=4.4 \mathrm{~Hz}, 1 \mathrm{H}), 6.12(\mathrm{~d}, J=4.4 \mathrm{~Hz}, 1 \mathrm{H})$, $7.12(\mathrm{t}, J=0.8 \mathrm{~Hz}, 1 \mathrm{H}), 7.25-7.41(\mathrm{~m}, 5 \mathrm{H}), 7.47-7.51(\mathrm{~m}, 2 \mathrm{H}), 7.66-7.70(\mathrm{~m}, 1 \mathrm{H}), 7.75-7.79(\mathrm{~m}, 1 \mathrm{H})$. The ${ }^{1} \mathrm{H}$ NMR spectrum is in agreement with that in the literature. ${ }^{23}$

To a round-bottom flask were added benzo[b]thiophen-2-yl-phenylmethanol (2.01 g, $8.36 \mathrm{mmol}), \mathrm{NaBH}_{4}$ and $\mathrm{Et}_{2} \mathrm{O}(150 \mathrm{~mL})$. To the mixture was added $\mathrm{CF}_{3} \mathrm{COOH}(12 \mathrm{~mL}, 16 \mathrm{mmol})$ dropwise, and the mixture was stirred at room temperature for $6 \mathrm{~h}$. To the mixture was added $10 \mathrm{wt} \%$ aq $\mathrm{NaOH}(150 \mathrm{~mL})$, and the mixture was stirred at room temperature for $30 \mathrm{~min}$. The mixture was washed with $\mathrm{H}_{2} \mathrm{O}$ and brine, and dried over $\mathrm{Na}_{2} \mathrm{SO}_{4}$. After removal of the solvent under reduced pressure, the residue was purified by flash chromatography (hexane) to give 2-benzylbenzo[b]thiophene (6) in $80 \%$ yield ( $1.50 \mathrm{~g}, 6.69 \mathrm{mmol})$. TLC $\mathrm{R}_{f}$ 0.72 (hexane/EtOAc 5:1); ${ }^{1} \mathrm{H}$ NMR (400 MHz, $\mathrm{CDCl}_{3}$ ) $\delta 4.23(\mathrm{~s}, 2 \mathrm{H}), 6.99-7.01$ (m, 1H), 7.22-7.36 (m, 7 $\mathrm{H}), 7.64-7.67(\mathrm{~m}, 1 \mathrm{H}), 7.71-7.75(\mathrm{~m}, 1 \mathrm{H})$. The ${ }^{1} \mathrm{H}$ NMR spectrum is in agreement with that in the literature. $^{24}$ 
Preparation of 3-Acetyl-4-methoxytoluene (8)<smiles>COc1ccc(C)c(C(=O)O[Na])c1</smiles>

To a round-bottom flask were added $\mathrm{LiClO}_{4}(2.11 \mathrm{~g}, 19.8 \mathrm{mmol})$ and $\mathrm{Ac}_{2} \mathrm{O}(1 \mathrm{~mL}, 10.6 \mathrm{mmol})$. To the mixture was added 4-methoxytoluene $(1.25 \mathrm{~mL}, 9.92 \mathrm{mmol})$ dropwise at $100{ }^{\circ} \mathrm{C}$, and the mixture was stirred at $100{ }^{\circ} \mathrm{C}$ for $3 \mathrm{~h}$. Then, the mixture was diluted with $\mathrm{CH}_{2} \mathrm{Cl}_{2}$, washed with sat. $\mathrm{NaHCO}_{3}$ and $\mathrm{H}_{2} \mathrm{O}$, and dried over $\mathrm{Na}_{2} \mathrm{SO}_{4}$. After removal of the solvent under reduced pressure, the residue was purified by GPC to give 3-acetyl-4-methoxytoluene (8) in 77\% yield (1.12 g, $7.43 \mathrm{mmol}$ ). TLC $\mathrm{R}_{f} 0.33$ (hexane/EtOAc 5:1); ${ }^{1} \mathrm{H}$ NMR (400 MHz, $\left.\mathrm{CDCl}_{3}\right) \delta 2.30(\mathrm{~s}, 3 \mathrm{H}), 2.61(\mathrm{~s}, 3 \mathrm{H}), 3.88(\mathrm{~s}, 3 \mathrm{H}), 6.86(\mathrm{~d}, J=8.4 \mathrm{~Hz}, 1 \mathrm{H}), 7.26(\mathrm{dd}, J=1.8$, $8.4 \mathrm{~Hz}, 1 \mathrm{H}), 7.53(\mathrm{~d}, J=2.2 \mathrm{~Hz}, 1 \mathrm{H}) ;{ }^{13} \mathrm{C} \mathrm{NMR}\left(100 \mathrm{MHz}, \mathrm{CDCl}_{3}\right) \delta 20.2,31.8,55.5,111.5,127.8,129.8$, 130.6, 134.1, 156.9, 200.1; HRMS (ESI) calcd for $\mathrm{C}_{10} \mathrm{H}_{12} \mathrm{O}_{2}\left(\mathrm{M}+\mathrm{H}^{+}\right)$: 165.0910, found: 165.0907.

\section{Synthesis of 2-Benzyl-3-(3-acetyl-4-methoxybenzyl)benzo[b]thiophene (9)}

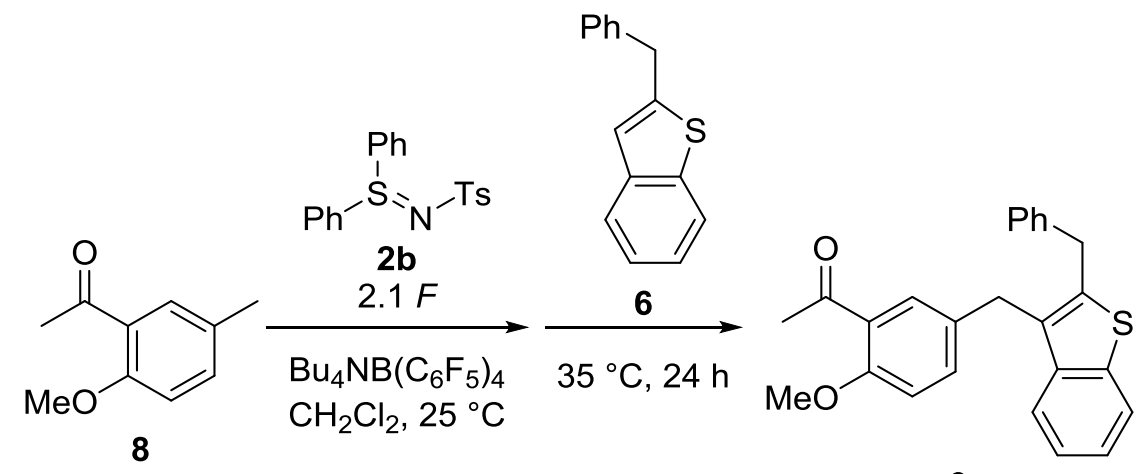

9

In the anodic chamber were placed 3-acetyl-4-methoxytoluene (8) (15.4 mg, $0.094 \mathrm{mmol})$, $S, S$-diphenyl- $N$-(4-methylbenzenesulfonyl)sulfilimine $\quad(\mathbf{2 b}) \quad(351 \quad \mathrm{mg}, \quad 0.99 \quad \mathrm{mmol}), \quad$ and $\quad 0.1 \quad \mathrm{M}$ $\mathrm{Bu}_{4} \mathrm{NB}\left(\mathrm{C}_{6} \mathrm{~F}_{5}\right)_{4} / \mathrm{CH}_{2} \mathrm{Cl}_{2}(10 \mathrm{~mL})$. In the cathodic chamber were placed $\mathrm{TfOH}(30 \mu \mathrm{L}, 0.68 \mathrm{mmol})$ and $0.1 \mathrm{M}$ $\mathrm{Bu}_{4} \mathrm{NB}\left(\mathrm{C}_{6} \mathrm{~F}_{5}\right)_{4} / \mathrm{CH}_{2} \mathrm{Cl}(10 \mathrm{~mL})$. The constant current electrolysis $(4.0 \mathrm{~mA})$ was carried out at $25{ }^{\circ} \mathrm{C}$ with magnetic stirring until $2.1 F$ of electricity was consumed. The solution in the anodic chamber was transferred to a $100 \mathrm{~mL}$ round-bottom flask under argon atmosphere. 2-Benzylbenzo[b]thiophene (6) (219 $\mathrm{mg}$ ) was added to the solution, and the resulting mixtures were stirred at $35{ }^{\circ} \mathrm{C}$ for $24 \mathrm{~h}$. The solvent was removed under reduced pressure. The crude product was purified by flash chromatography (hexane/EtOAc 100:0 to 10:1) and GPC to obtain 2-benzyl-3-(3-acetyl-4-methoxybenzyl)benzo[b]thiophene (9) in $70 \%$ yield (25.5 $\mathrm{mg}, 0.066 \mathrm{mmol}$ ). TLC $\mathrm{R}_{f} 0.32$ (hexane/EtOAc 5:1); ${ }^{1} \mathrm{H} \mathrm{NMR}\left(400 \mathrm{MHz}, \mathrm{CDCl}_{3}\right) \delta 2.56(\mathrm{~s}, 3 \mathrm{H}), 3.83$ (s, 3H), $4.17(\mathrm{~s}, 2 \mathrm{H}), 4.22(\mathrm{~s}, 2 \mathrm{H}), 6.77(\mathrm{~d}, J=8.8 \mathrm{~Hz}, 1 \mathrm{H}), 7.07(\mathrm{dd}, J=2.6,8.8 \mathrm{~Hz}, 1 \mathrm{H}), 7.19-7.30(\mathrm{~m}, 7 \mathrm{H})$, 7.49-7.54 (m, 1H), $7.61(\mathrm{~d}, J=2.6 \mathrm{~Hz}, 1 \mathrm{H}), 7.71-7.75(\mathrm{~m}, 1 \mathrm{H}) ;{ }^{13} \mathrm{C} \mathrm{NMR}\left(100 \mathrm{MHz}, \mathrm{CDCl}_{3}\right) \delta 31.0,31.7$, 34.4, 55.4, 111.7, 121.8, 122.1, 123.7, 123.9, 126.5, 127.9, 128.46, 128.50, 129.7, 129.8, 131.5, 132.9, 138.8, 139.3, 140.00, 140.04, 157.3, 199.6; HRMS (EI) calcd for $\mathrm{C}_{25} \mathrm{H}_{23} \mathrm{O}_{2} \mathrm{~S}\left(\mathrm{M}+\mathrm{H}^{+}\right)$: 387.1413, found: 387.1407 . 
Synthesis of 2-Benzyl-3-(3-acetyl-4-hydroxybenzyl)benzo[b]thiophene $(7)^{22}$<smiles>COc1ccc(Cc2c(Cc3ccccc3)sc3ccccc23)cc1C(C)=O</smiles>

9

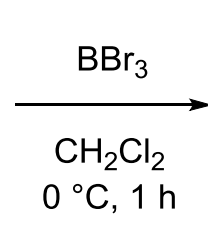

$0{ }^{\circ} \mathrm{C}, 1 \mathrm{~h}$<smiles>CC(=O)c1cc(Cc2c(Cc3ccccc3)sc3ccccc23)ccc1O</smiles>

7

To a round-bottom flask were added 2-benzyl-3-(3-acetyl-4-methoxybenzyl)benzo[b]thiophene (9) (56.2 $\mathrm{mg}, 0.145 \mathrm{mmol})$ and $\mathrm{CH}_{2} \mathrm{Cl}_{2}(2 \mathrm{~mL}) . \mathrm{BBr}_{3}\left(1.0 \mathrm{M}\right.$ in $\left.\mathrm{CH}_{2} \mathrm{Cl}_{2}, 0.60 \mathrm{~mL}\right)$ was added at $0{ }^{\circ} \mathrm{C}$. After being stirred at $0{ }^{\circ} \mathrm{C}$ for $1 \mathrm{~h}, \mathrm{H}_{2} \mathrm{O}(5 \mathrm{~mL})$ was added to the mixture. After being stirred at room temperature for 30 min, the mixture was washed with $\mathrm{H}_{2} \mathrm{O}$, dried over $\mathrm{Na}_{2} \mathrm{SO}_{4}$. The solvent was removed under reduced pressure to give 2-benzyl-3-(3-acetyl-4-hydroxybenzyl)benzo[b]thiophene (7) in 99\% yield (53.4 $\mathrm{mg}$, $0.143 \mathrm{mmol}$ ). TLC $\mathrm{R}_{f} 0.42$ (hexane/EtOAc 5:1); ${ }^{1} \mathrm{H}$ NMR (400 MHz, $\left.\mathrm{CDCl}_{3}\right) \delta 2.40$ (s, 3H), $4.18(\mathrm{~s}, 2 \mathrm{H}$ ), $4.23(\mathrm{~s}, 2 \mathrm{H}), 6.84(\mathrm{~d}, J=8.3 \mathrm{~Hz}, 1 \mathrm{H}), 7.18-7.32(\mathrm{~m}, 8 \mathrm{H}), 7.35(\mathrm{~d}, J=1.8 \mathrm{~Hz}, 1 \mathrm{H}), 7.50-7.54(\mathrm{~m}, 1 \mathrm{H})$, 7.77-7.80 (m, 1H), $12.10(\mathrm{~s}, 1 \mathrm{H}) ;{ }^{13} \mathrm{C} \mathrm{NMR}\left(100 \mathrm{MHz}, \mathrm{CDCl}_{3}\right) \delta 26.5,31.1,34.5,118.4,119.4,121.7,122.3$, 123.9, 124.1, 126.6, 128.5, 128.6, 129.5, 129.66, 129.74, 136.4, 138.9, 139.3, 139.9, 140.1, 160.7, 204.4; HRMS (EI) calcd for $\mathrm{C}_{24} \mathrm{H}_{21} \mathrm{O}_{2} \mathrm{~S}\left(\mathrm{M}+\mathrm{H}^{+}\right): 373.1257$, found: 373.1251 . 


\section{References}

(1) Saito, K.; Ueoka, K.; Matsumoto, K.; Suga, S.; Nokami, T.; Yoshida, J. Angew. Chem., Int. Ed. 2011, 50, 5153.

(2) Huang, S. L.; Swern, D. J. Org. Chem. 1978, 43, 4537.

(3) Marzinzik, A. L.; Sharpless, K. B. J. Org. Chem. 2001, 66, 594.

(4) Murigi, F. N.; Nichol, G. S.; Mash, E. A. J. Org. Chem. 2010, 75, 1293.

(5) Schneider, M. R.; Angerer, E.; Schonenberger, H.; Michel, R. T.; Fortmeyer, H. P. J. Med. Chem. 1982, 25, 1070 .

(6) Yu, J.-W.; Mao, S.; Wang, Y.-Q. Tetrahedron Lett. 2015, 56, 1575.

(7) Zhou, L.; Shi, Y.; Xian, Q.; Liu, Y.; Ye, F.; Zhang, Y.; Wang, J. Org. Lett. 2011, 13, 968.

(8) After electrolysis, the solution in the anodic chamber was transferred to a $100 \mathrm{~mL}$ round-bottom flask under argon atmosphere.

(9) Xian, T.; Dong, X.; Zhou, L. Org. Biomol. Chem. 2013, 11, 1490.

(10) Ashikari, Y.; Shimizu, A.; Nokami, T.; Yoshida, J. J. Am. Chem. Soc. 2013, 135, 16070.

(11) Okajima, M.; Soga, K.; Nokami, T.; Suga, S.; Yoshida, J. Org. Lett. 2006, 8, 5005.

(12) Chu, X.-Q.; Jiang, R.; Fang, Y.; Gu, Z.-Y.; Meng, H.; Wang, S.-Y.; Ji, S.-J. Tetrahedron Lett. 2013, 69, 1166.

(13) Kupracz, L.; Kirschning, A. J. Flow. Chem. 2012, 3, 11.

(14) Maity, P.; Shacklady-McAtee, D. M.; Yap, G. P. A.; Sirianni, E. R.; Watson, M. P. J. Am. Chem. Soc. 2013, 135, 280.

(15) Chem, D.; Xu, C.; Deng, J.; Jiang, C.; Wen, X.; Kong, L.; Zhang, J.; Sun, H. Tetrahedron 2014, 70, 1975.

(16) Bartoli, G.; Dalpozzo, R.; Nino, A. D.; Maiuolo, L.; Nardi, M.; Procopio, A.; Tagarelli, A. Eur. J. Org. Chem. 2004, 2176.

(17) Marjan, J.; Vrazic, D. Org. Biomol. Chem. 2013, 11, 1978.

(18) Nishimoto, Y.; Yasuda, M.; Baba, A. Org. Lett. 2007, 9, 4931.

(19) After electrolysis, the solution in the anodic chamber was transferred to a $100 \mathrm{~mL}$ round-bottom flask, the solvent was removed and 1,2-dichloromethane $(5 \mathrm{~mL})$ was added as solvent.

(20) Li, Y.; Hu, Y.-Y.; Zhang, S.-L. Chem. Commun. 2013, 49, 10635.

(21) Meyer, V. J.; Niggemann, M. Chem. Eur. J. 2012, 18, 4687.

(22) Shrestha, S.; Hwang, S. Y.; Lee, K.-H.; Cho, H. Bull. Korean Chem. Soc. 2005, 26, 1138.

(23) Kuriyama, M.; Ishiyama, N.; Shimazawa, R.; Onomura, O. Tetrahedron 2010, 66, 6814.

(24) Lapointe, D.; Fagnou, K. Org. Lett. 2009, 11, 4160.

(25) Bartoli, G.; Bosco, M.; Marcantoni, E.; Massaccesi, M.; Rinaldi, S.; Sambri, L. Tetrahedron Lett. 2002, 43,6331 . 
10. NMR Spectra
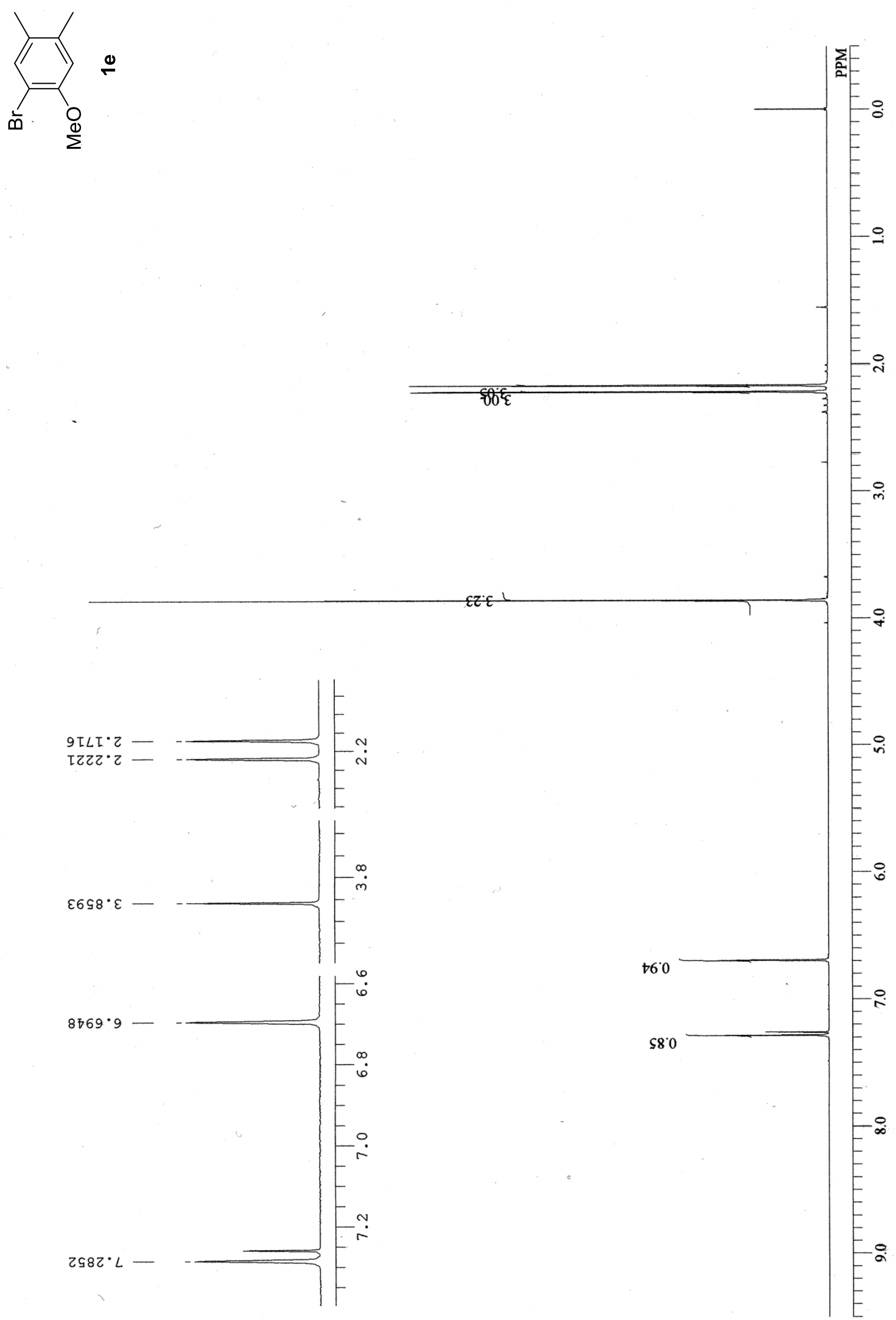

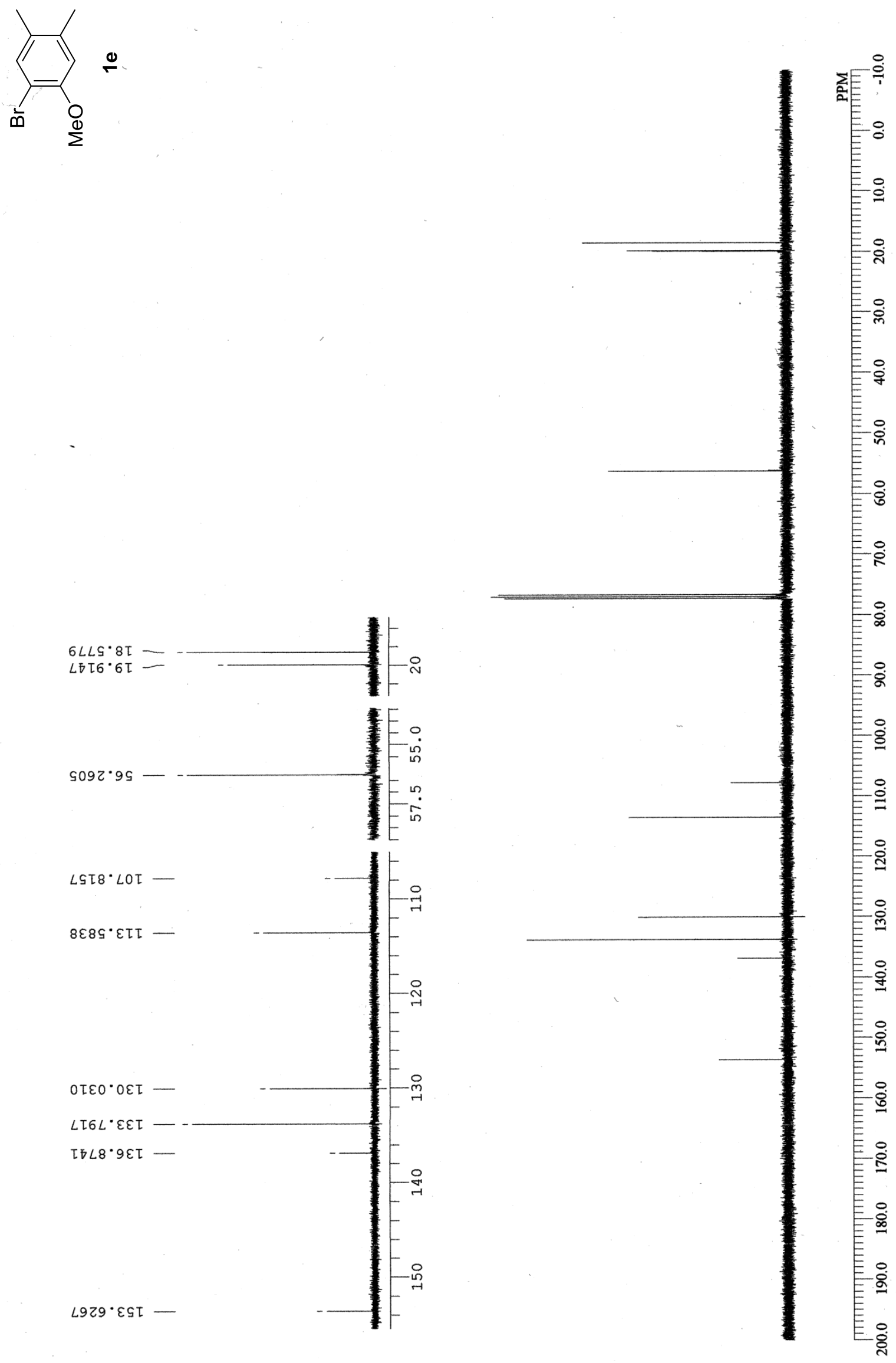


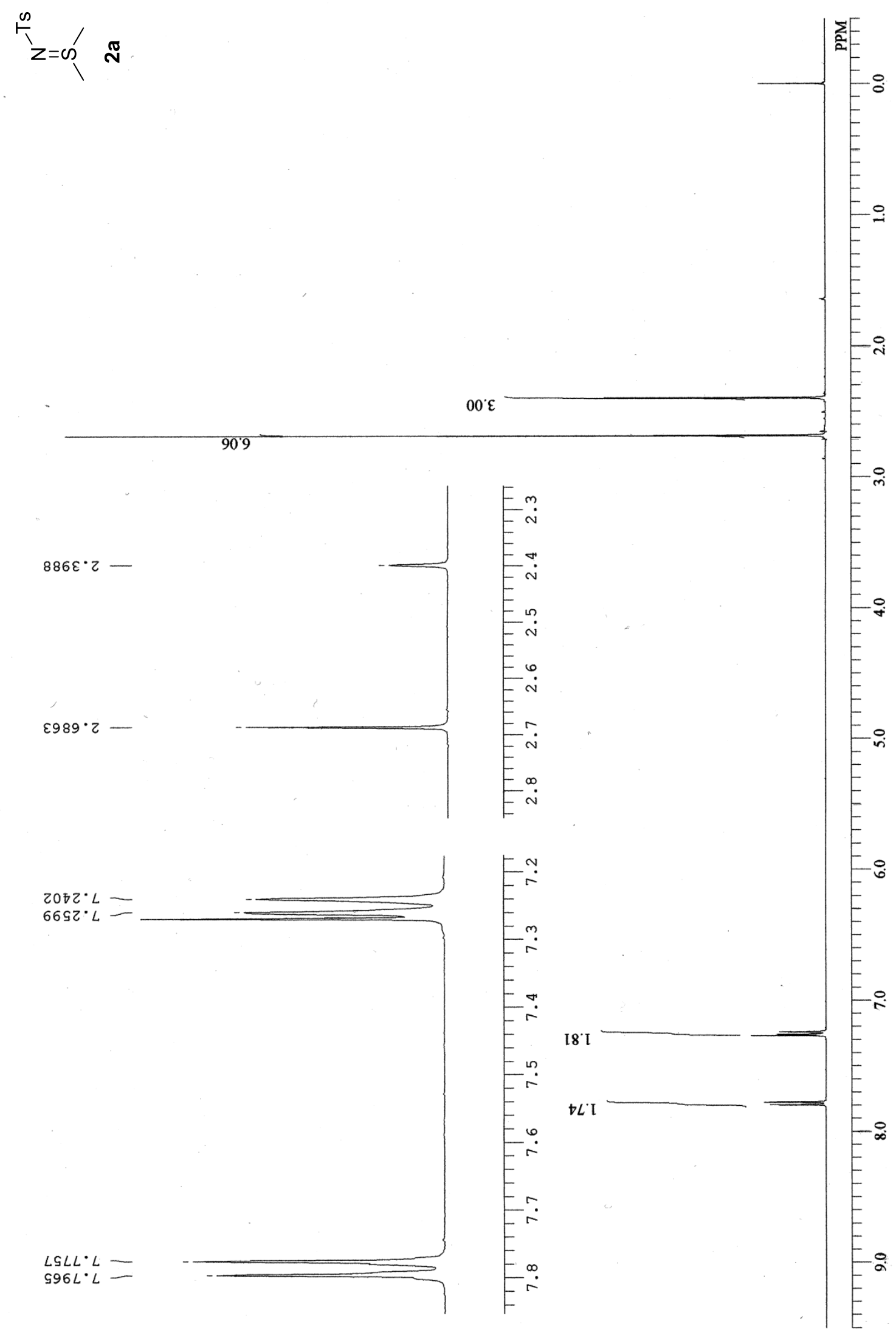



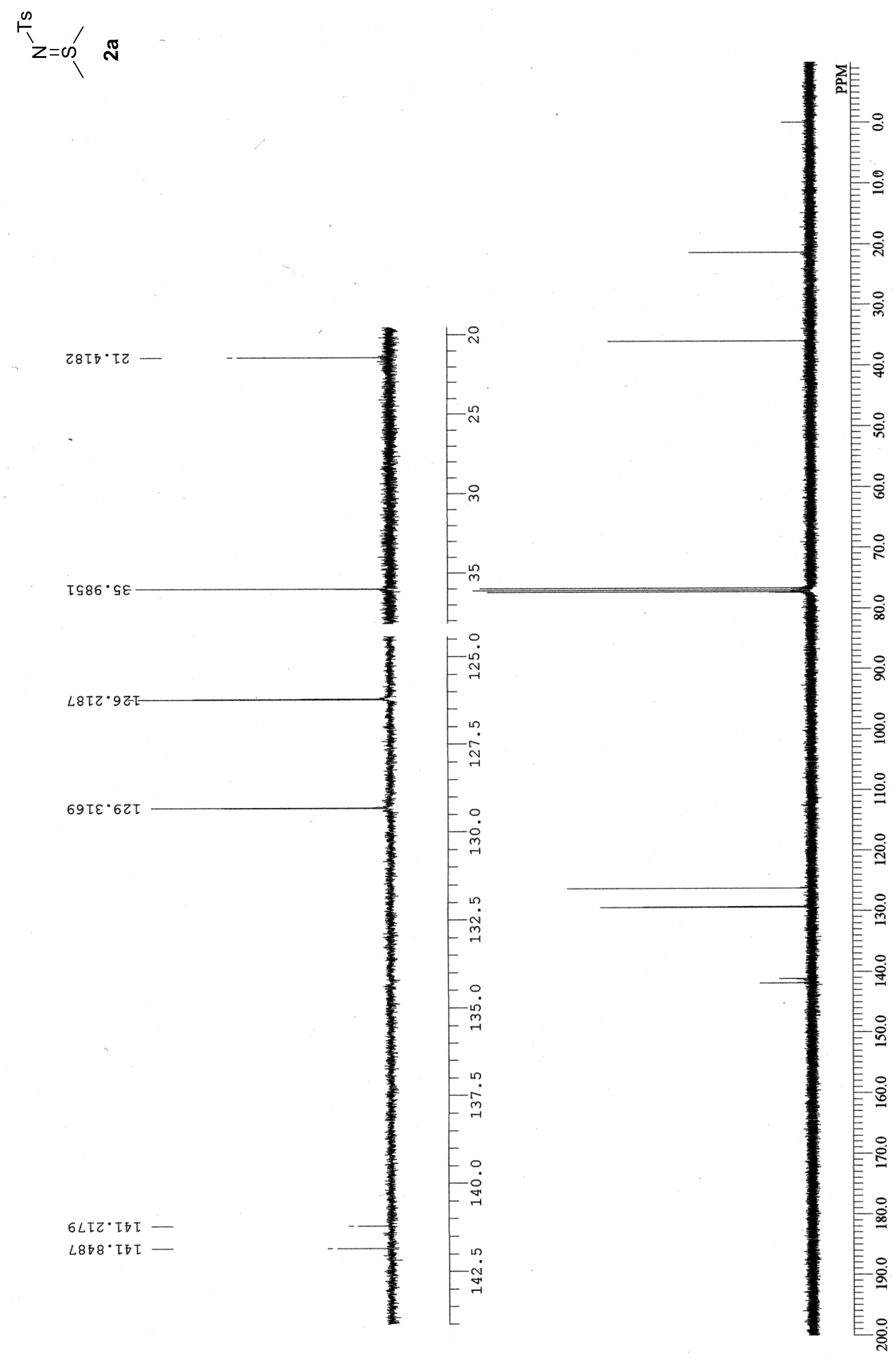

$6 L \tau{ }^{\circ} \tau \nabla \tau-$

$\angle 88^{\circ}$ TET

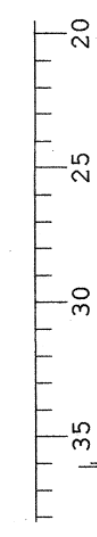

靠。

在。

웅

$\stackrel{8}{8}$

:

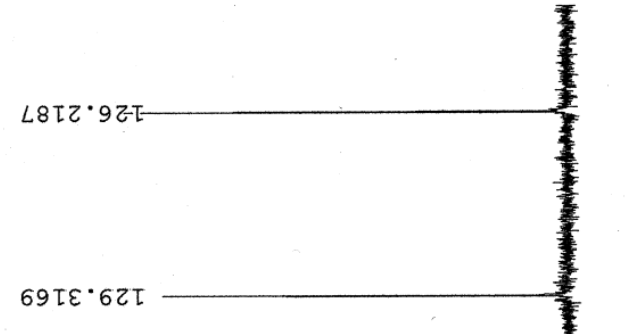

Fị.

E-

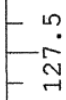

\begin{tabular}{l}
- \\
$E 0$ \\
\hline 0 \\
\hline 0 \\
\hline
\end{tabular}

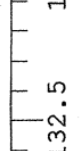

$=$

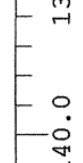

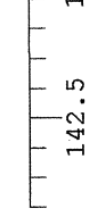

$\therefore$

:

\&.․․ 


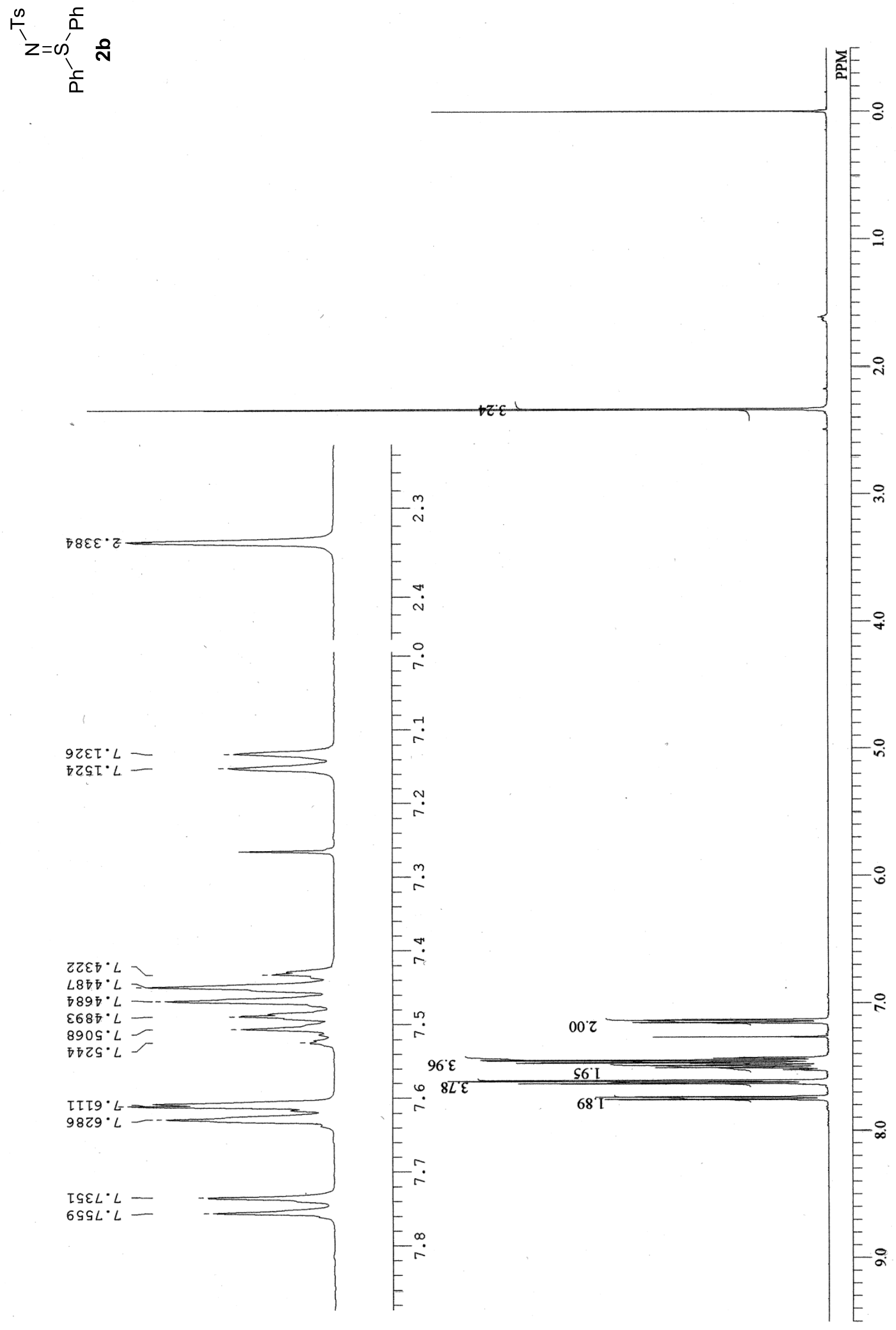




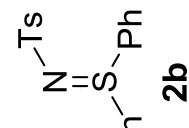

$$
\begin{aligned}
& \text { 듬 }
\end{aligned}
$$

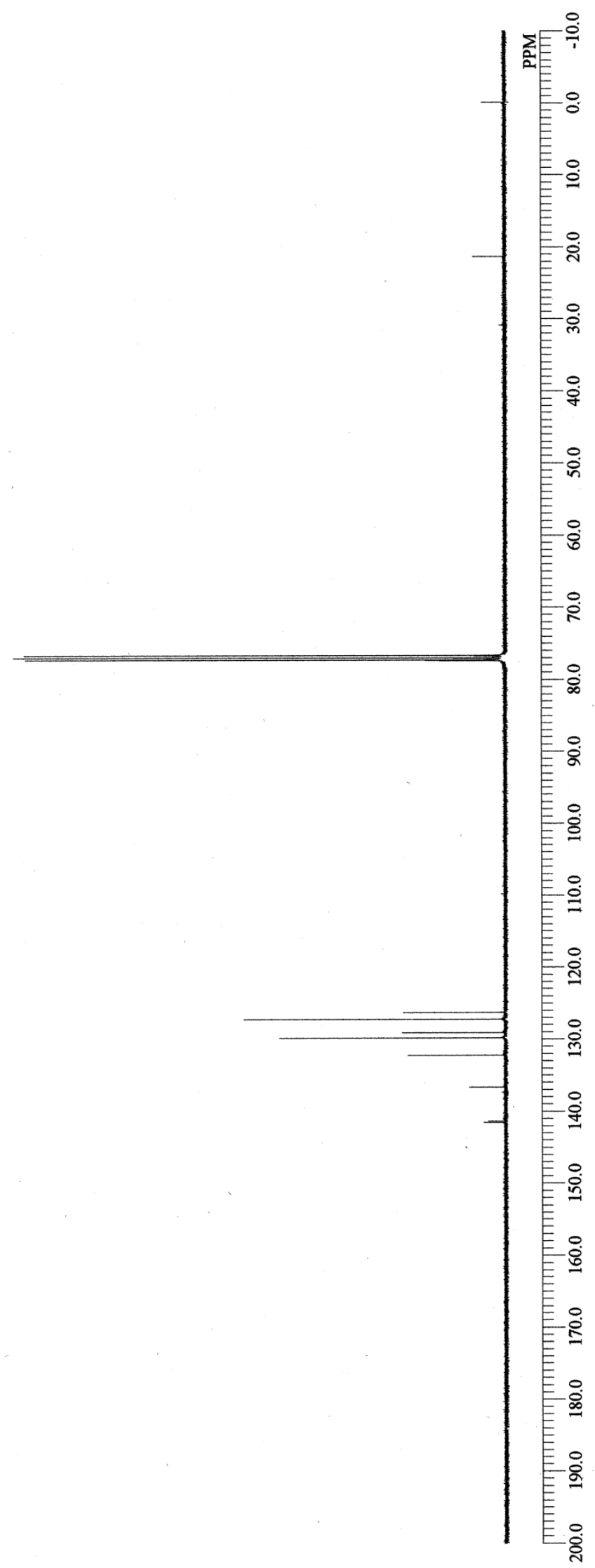




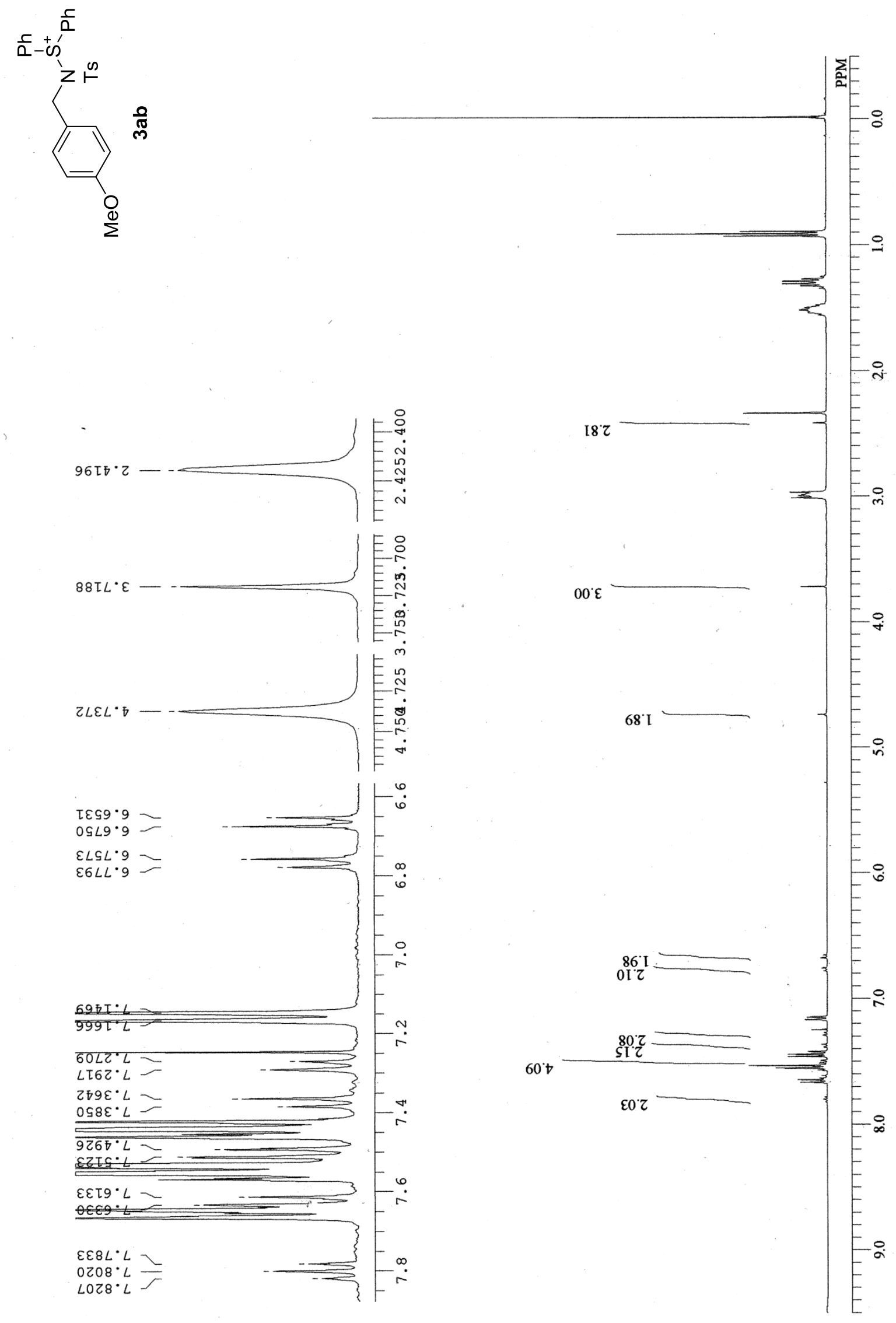



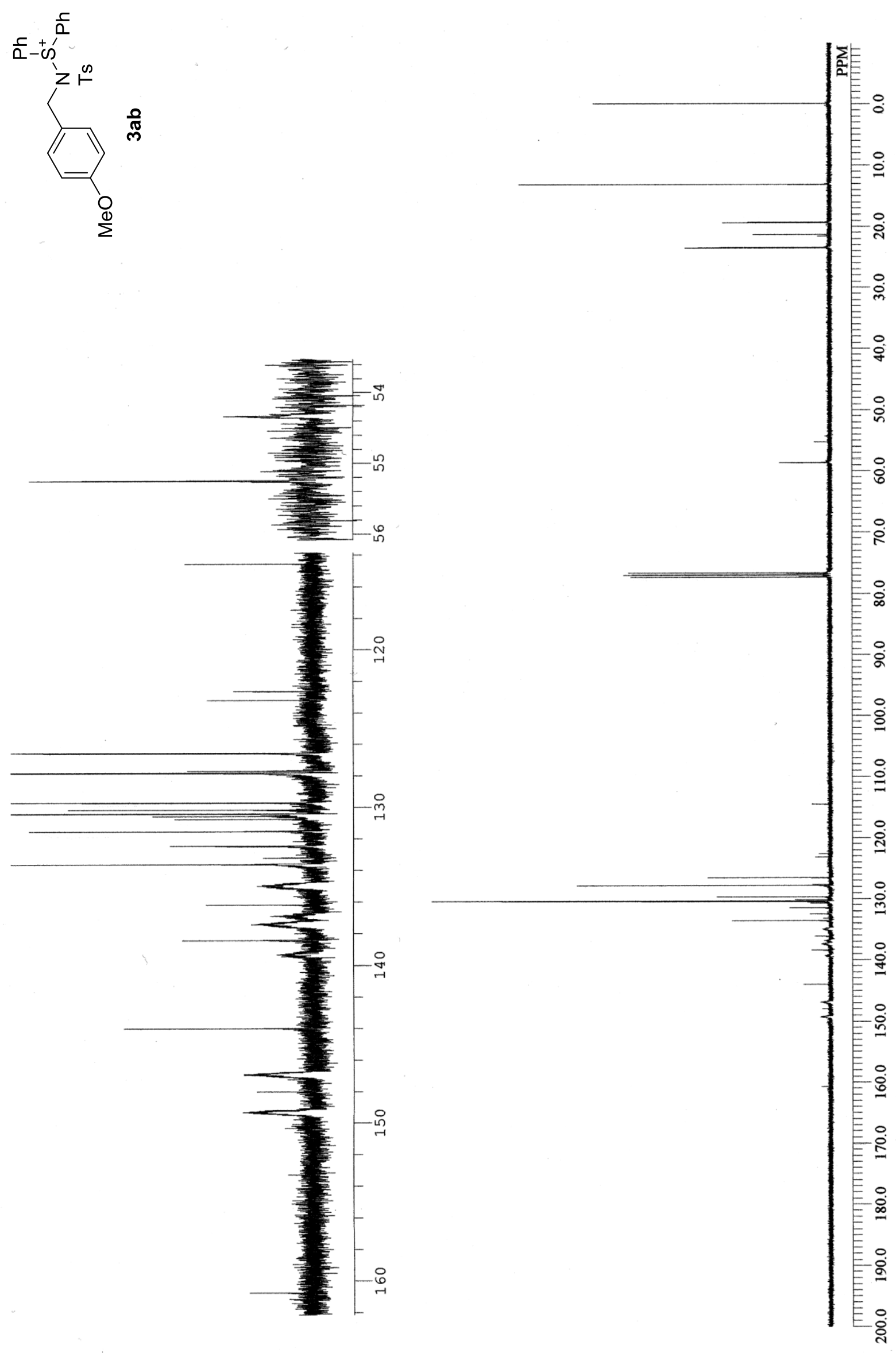


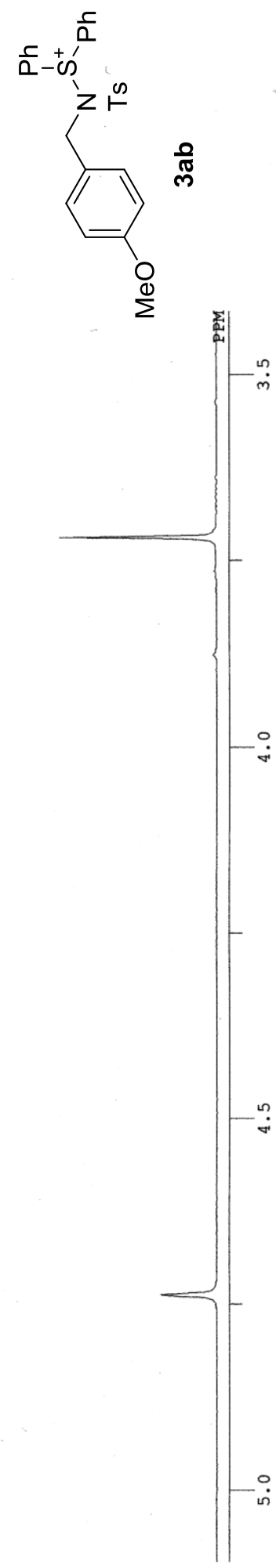

$\infty$

(ब)

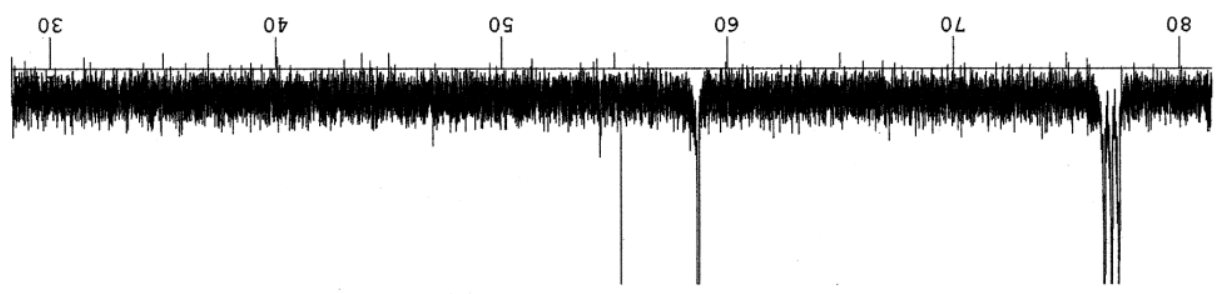


<smiles>COc1ccc(Cc2cc3ccccc3o2)cc1Br</smiles>

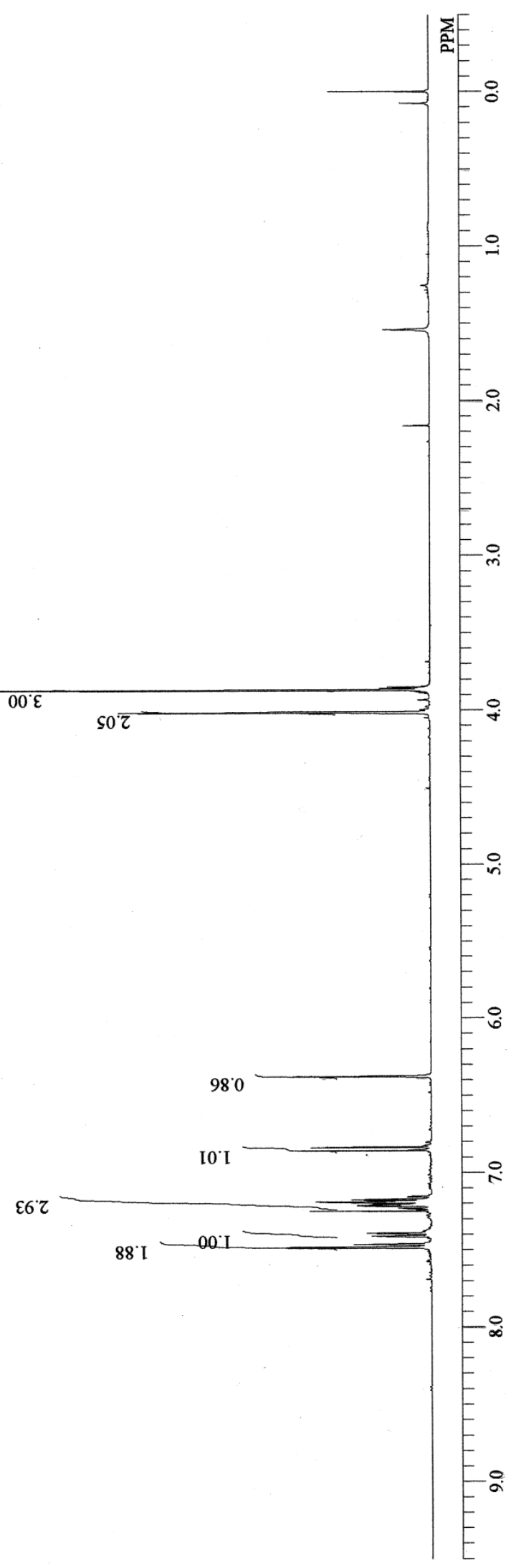



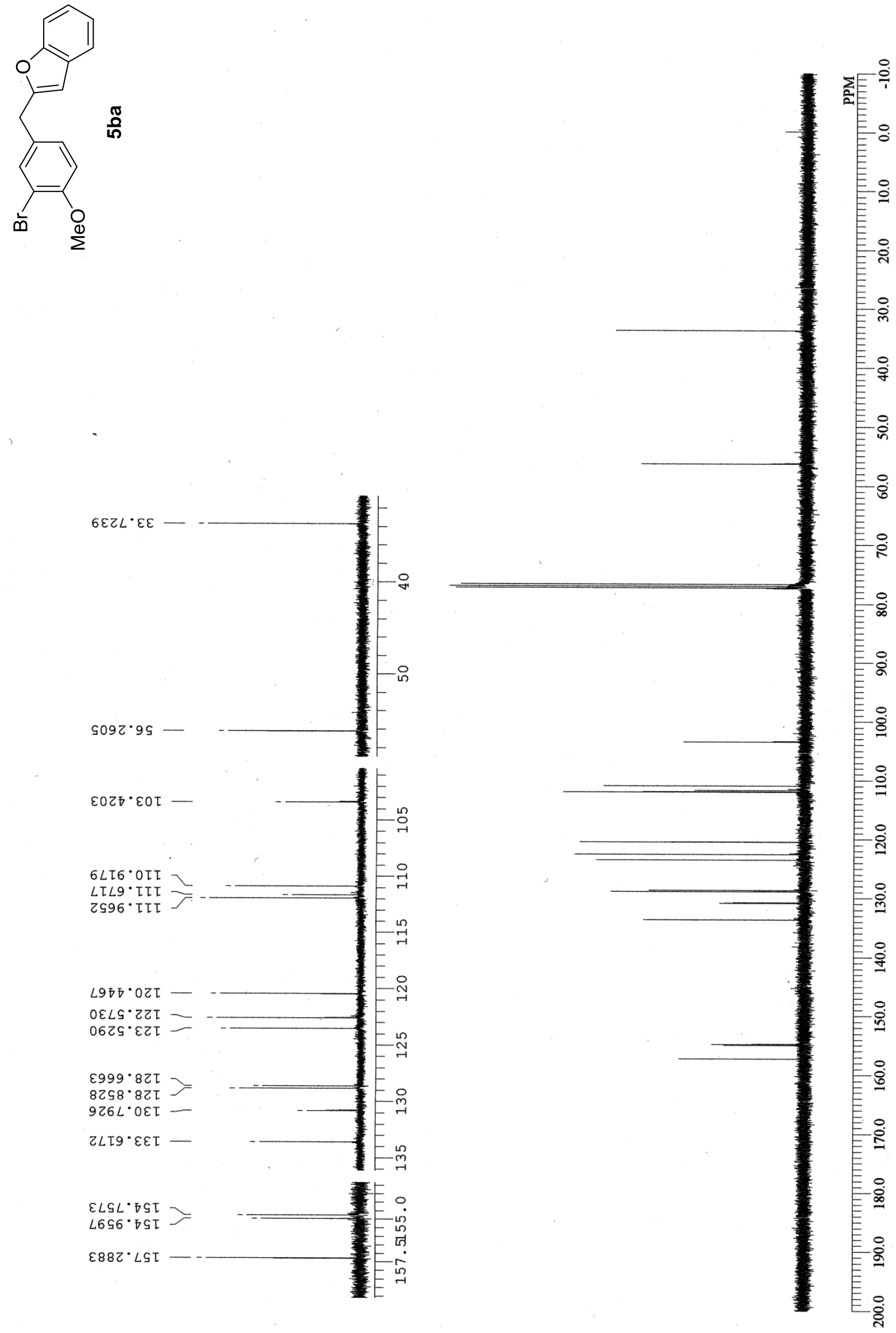

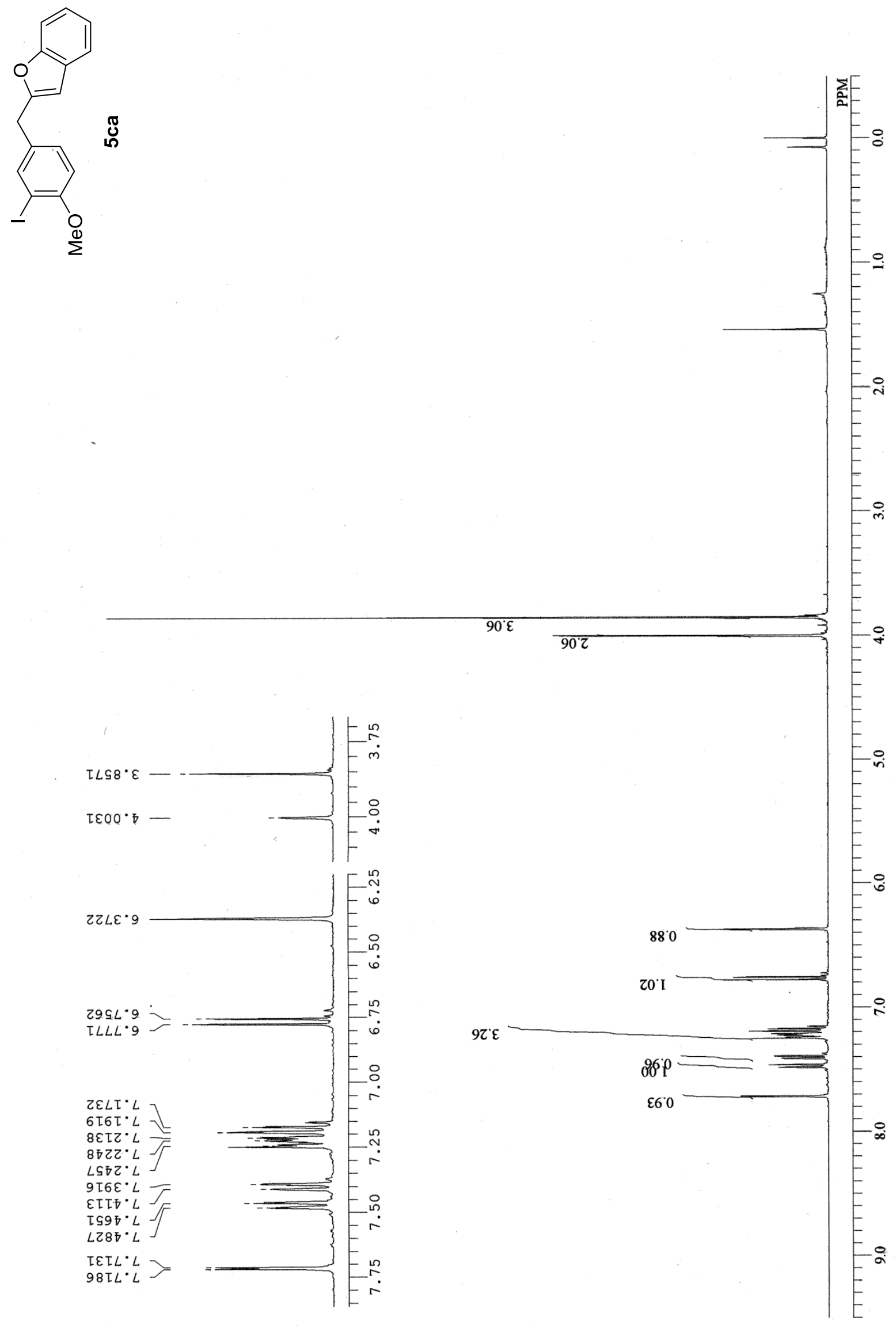

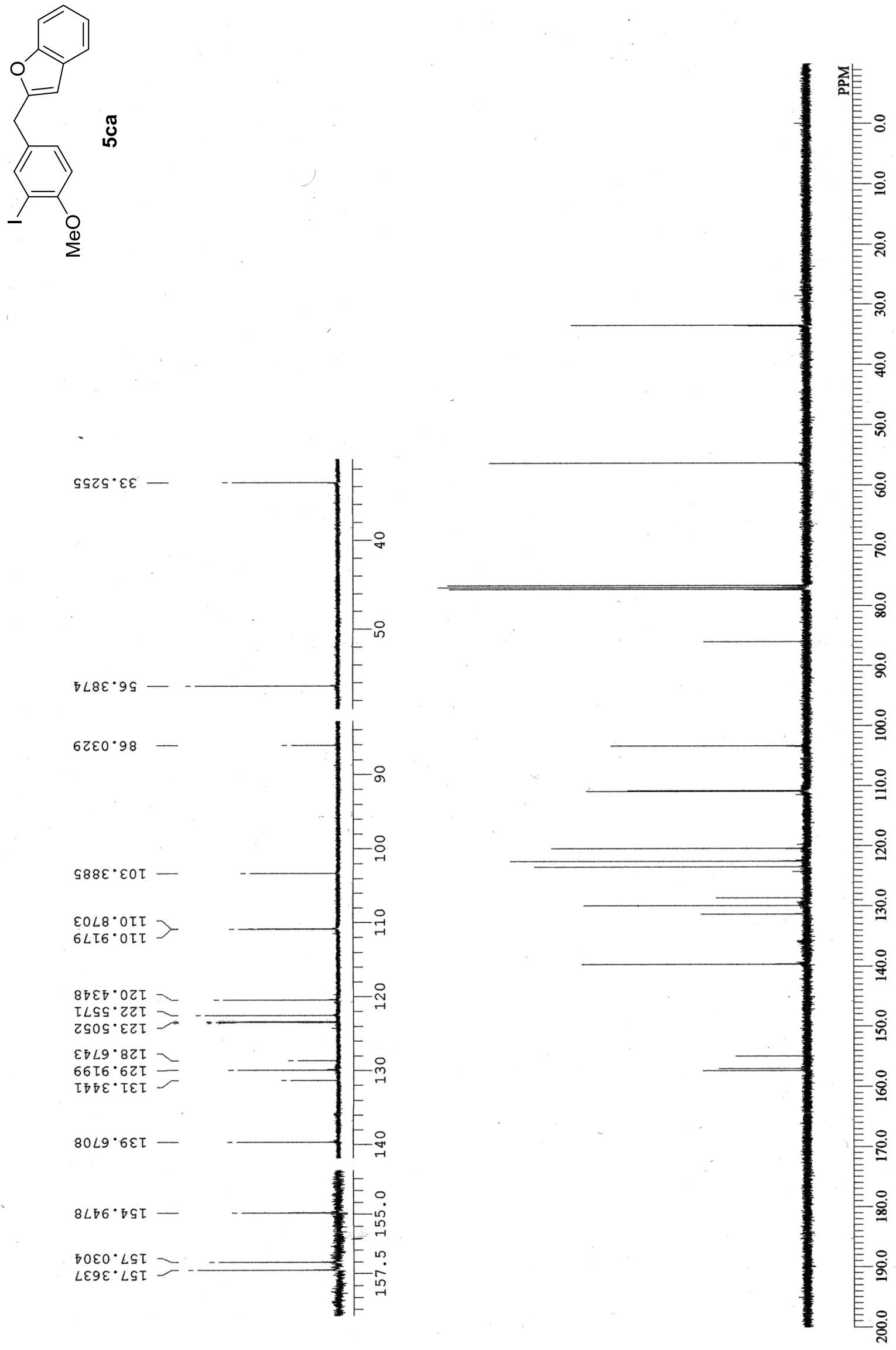

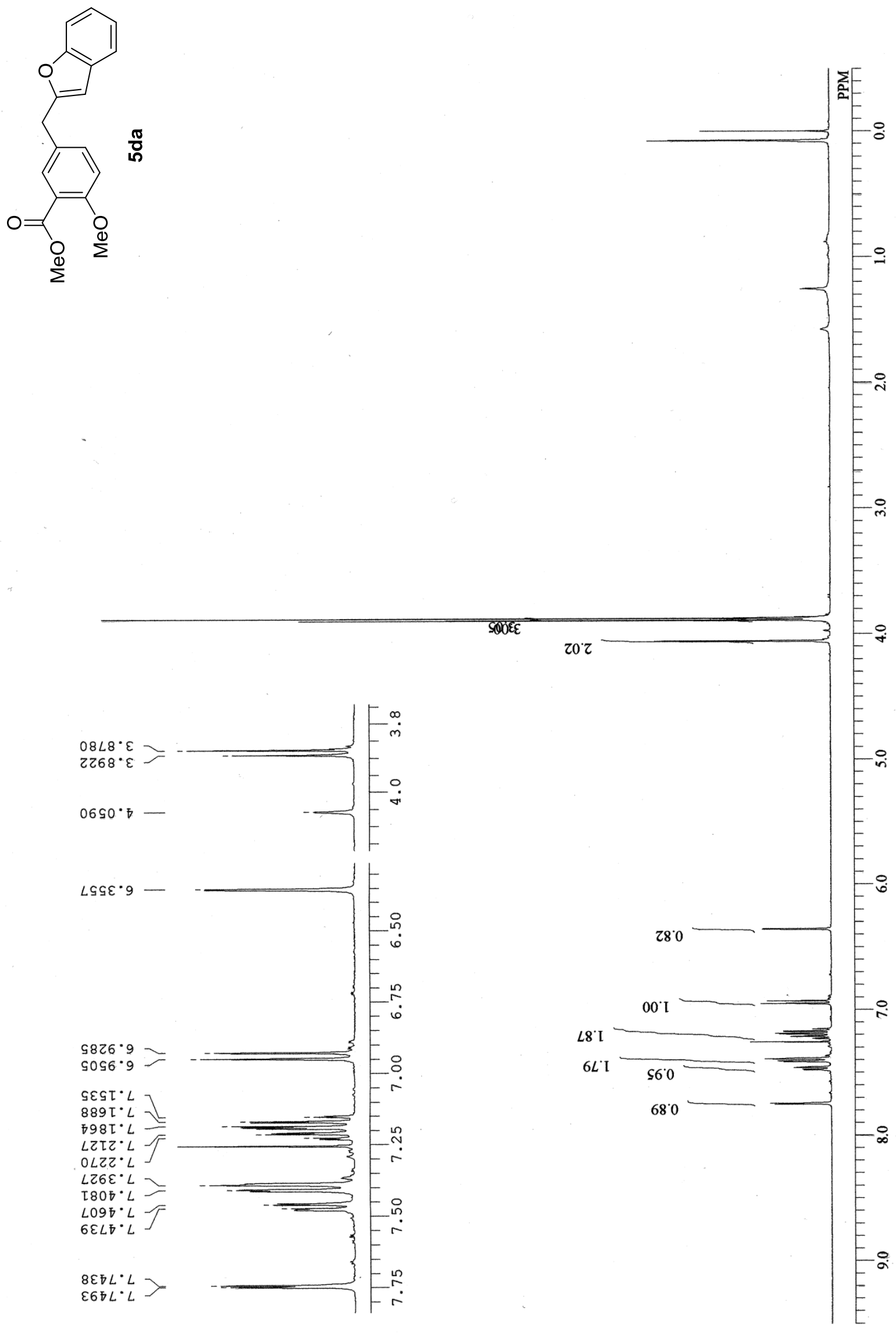

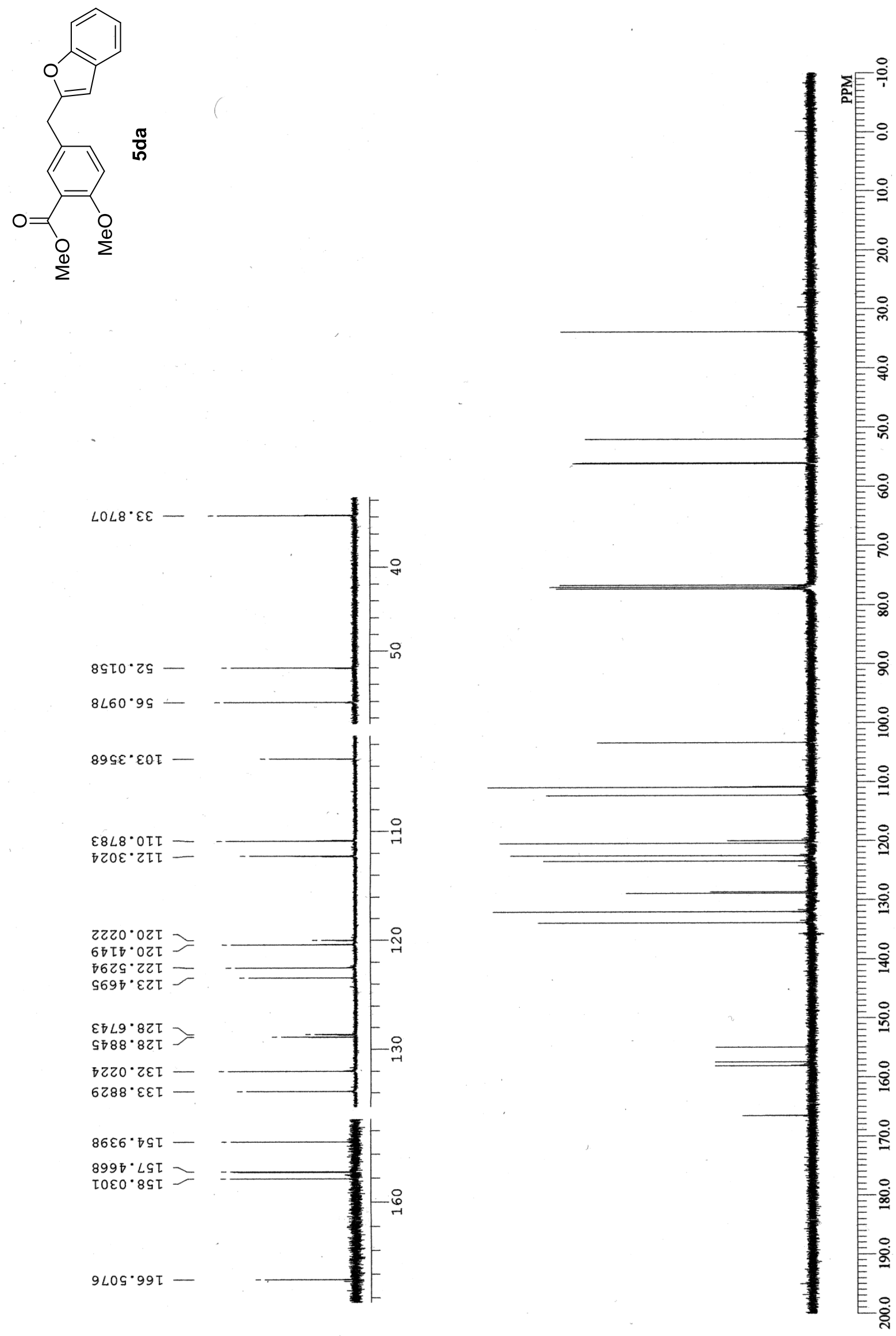

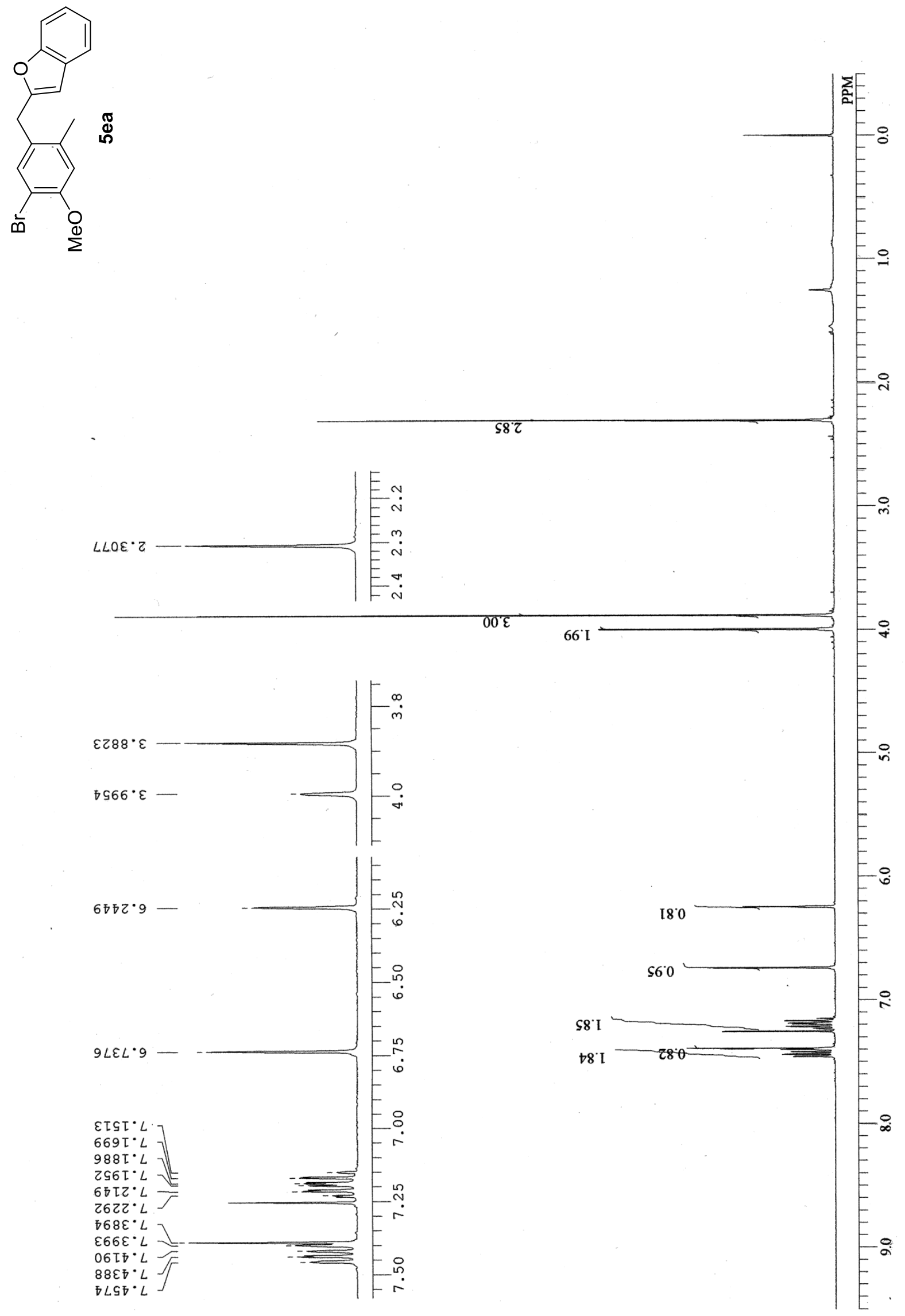

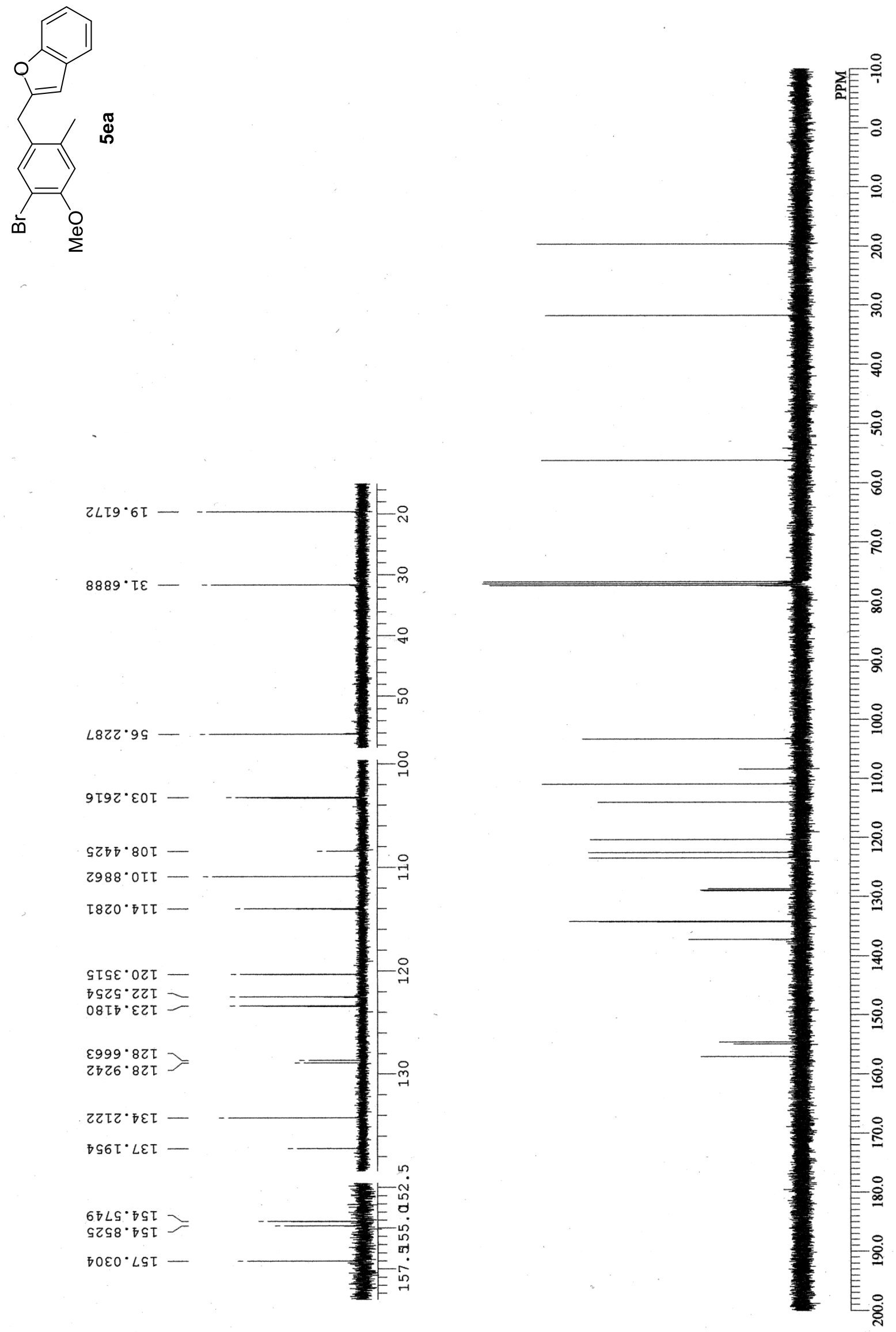
<smiles>COc1ccc(C(C(=O)O)c2cc3ccccc3o2)cc1</smiles>

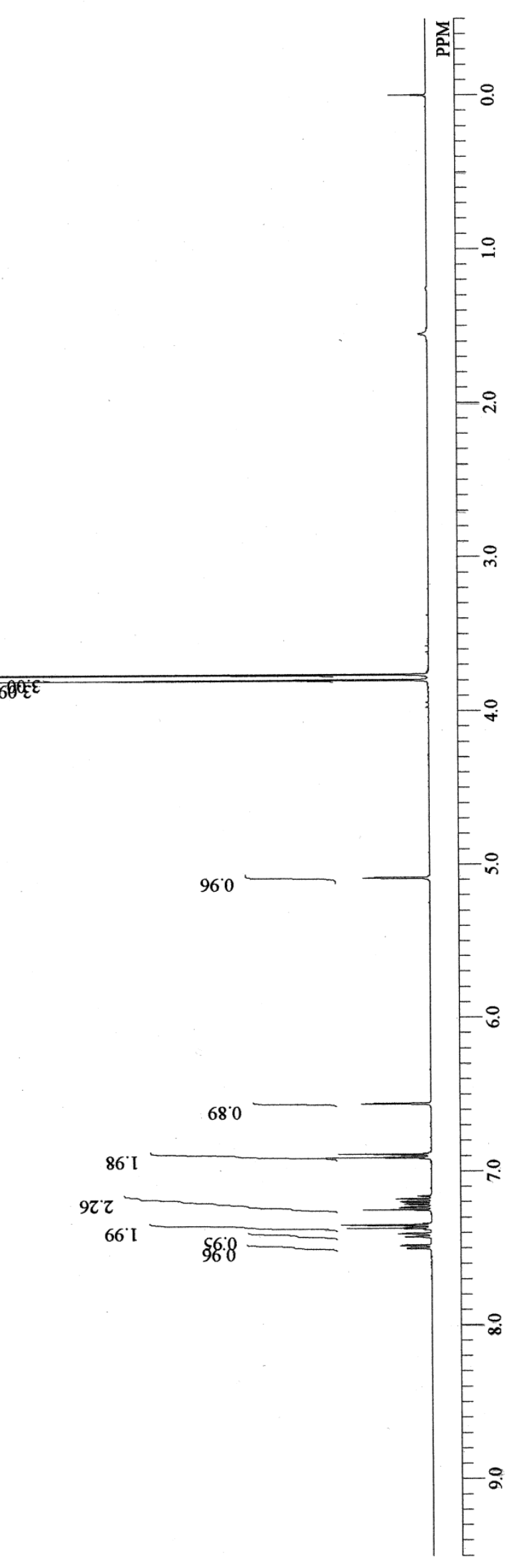


<smiles>COc1ccc(C(C(=O)O)c2cc3ccccc3o2)cc1</smiles>

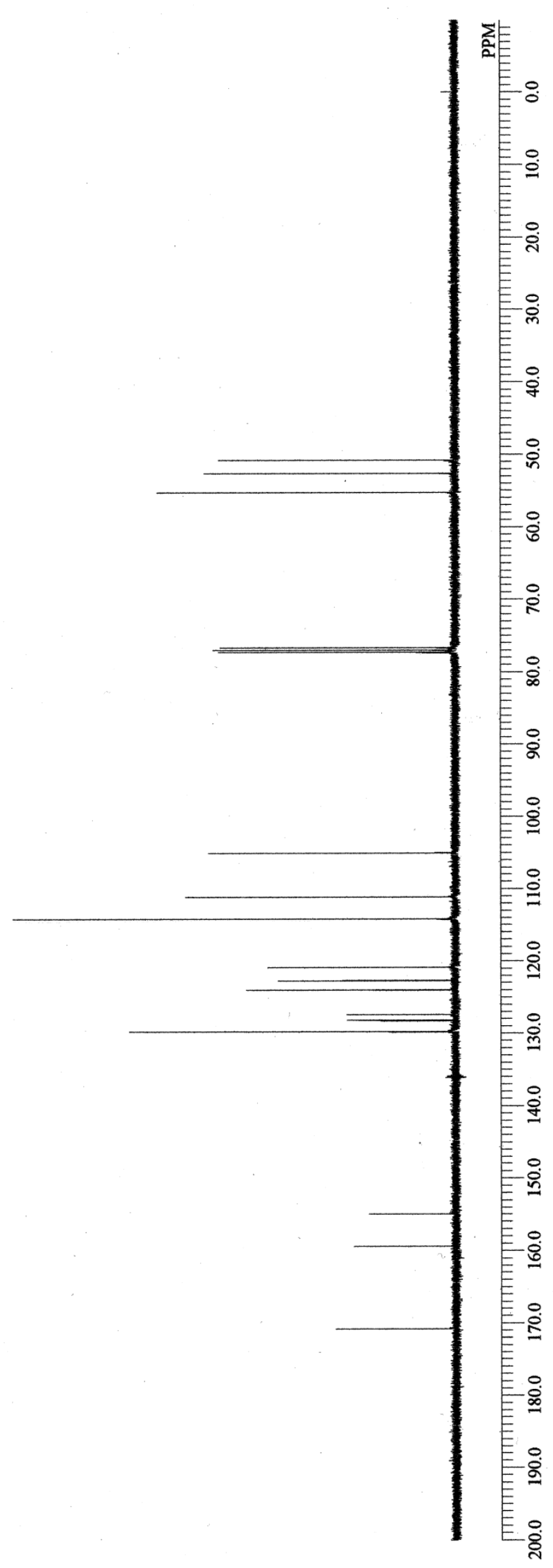


<smiles>COc1ccc(C(C(=O)[O-])c2cc3ccccc3o2)cc1</smiles>

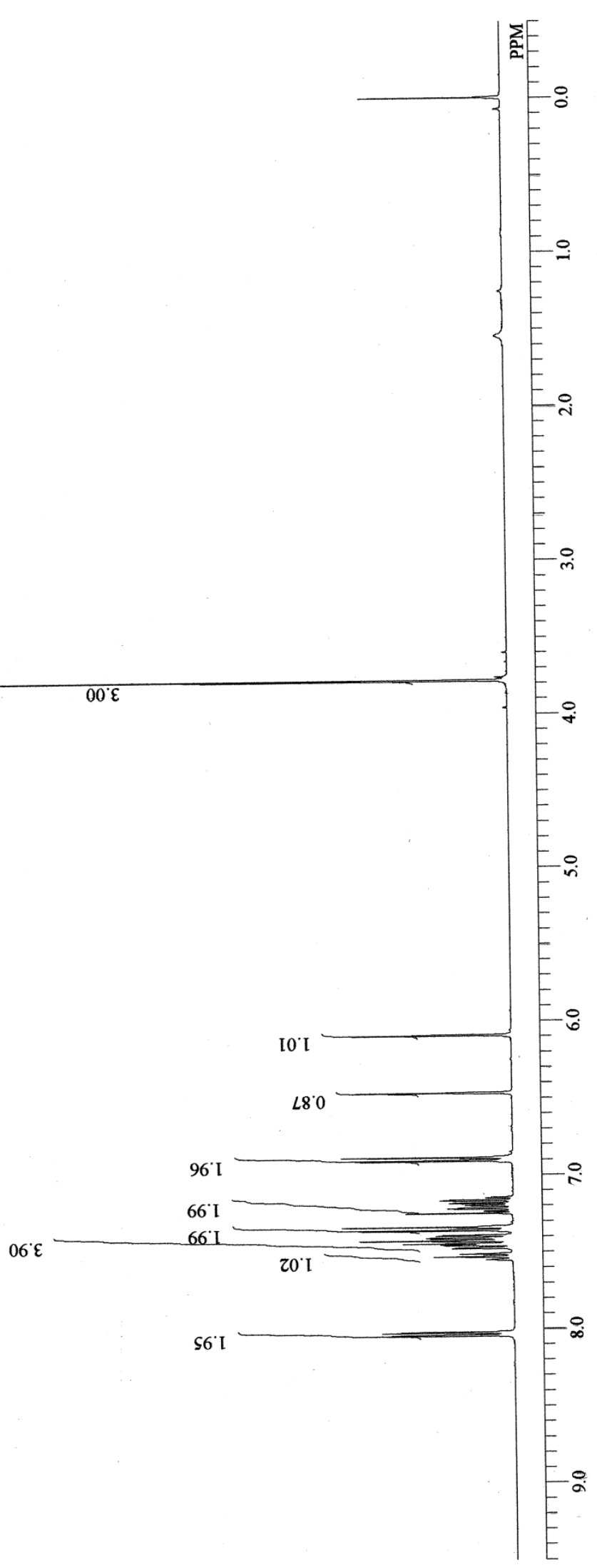


<smiles>COc1ccc(C(C(C)=O)c2cc3ccccc3o2)cc1</smiles>

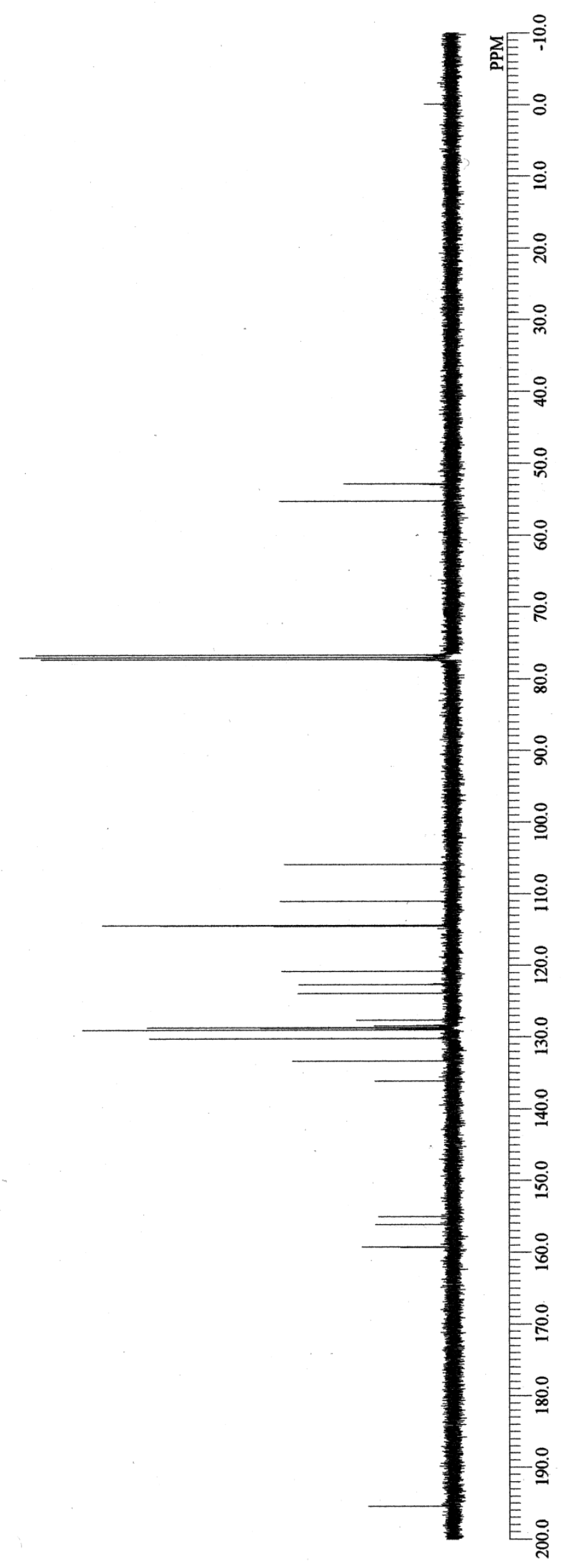



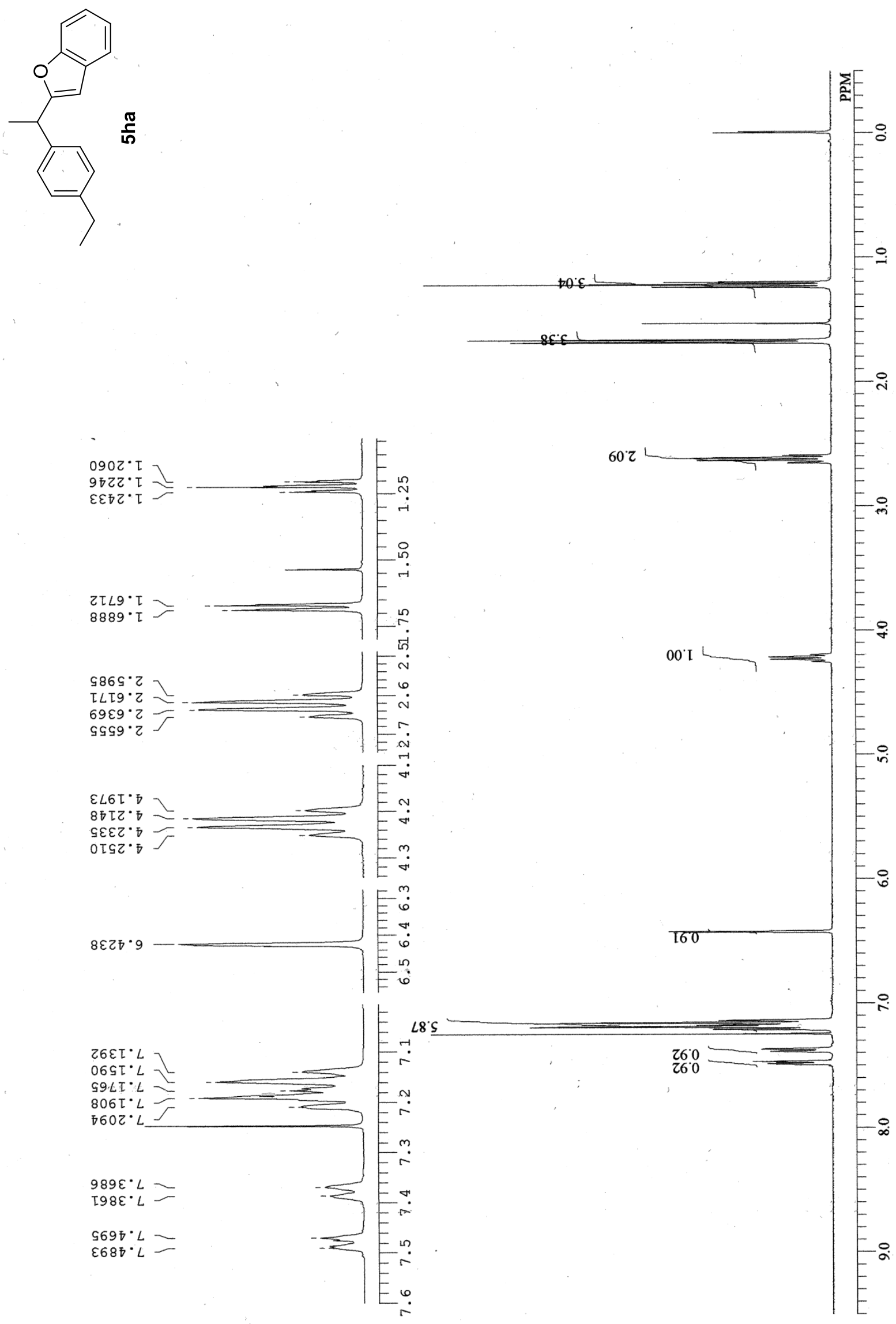

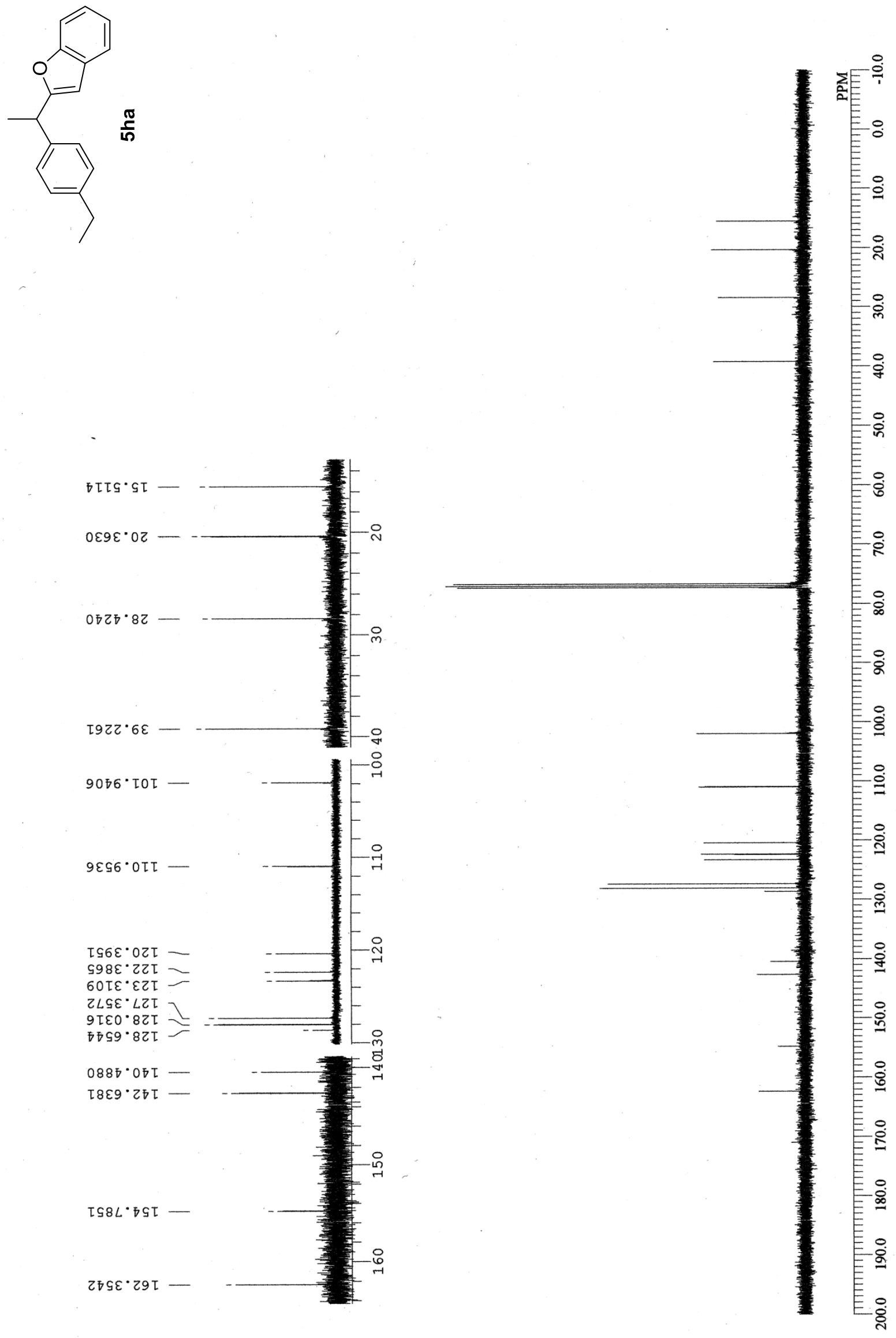

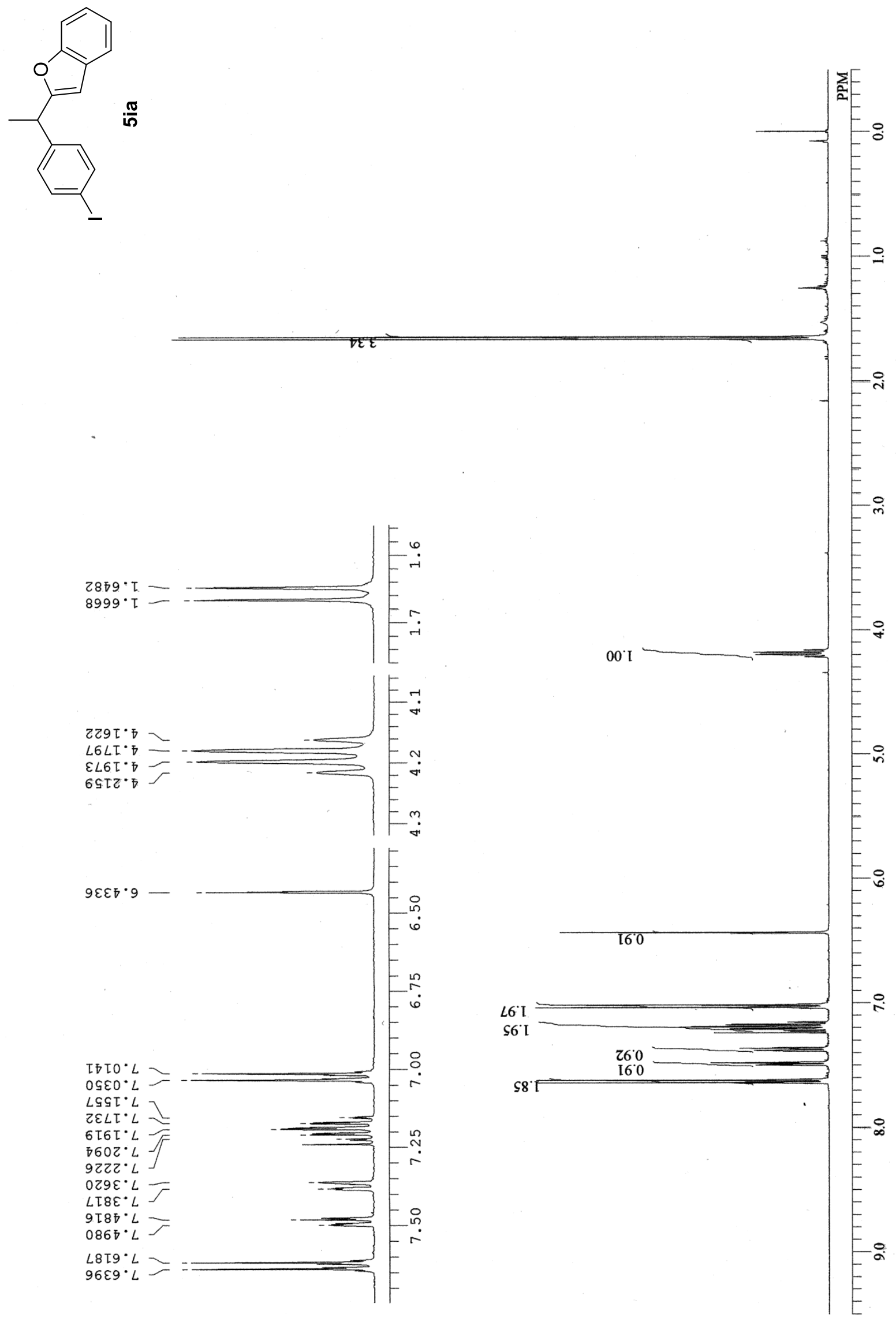

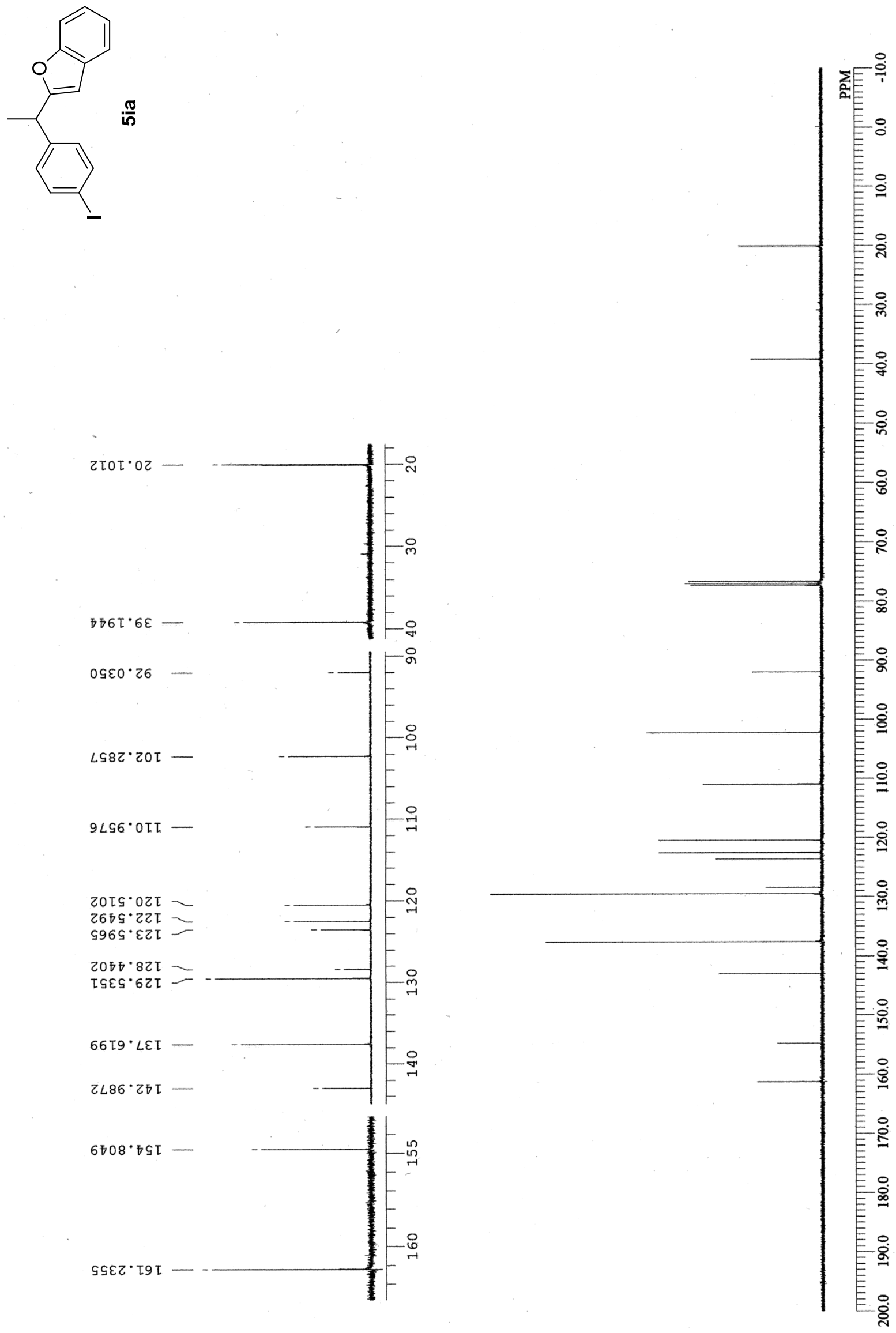

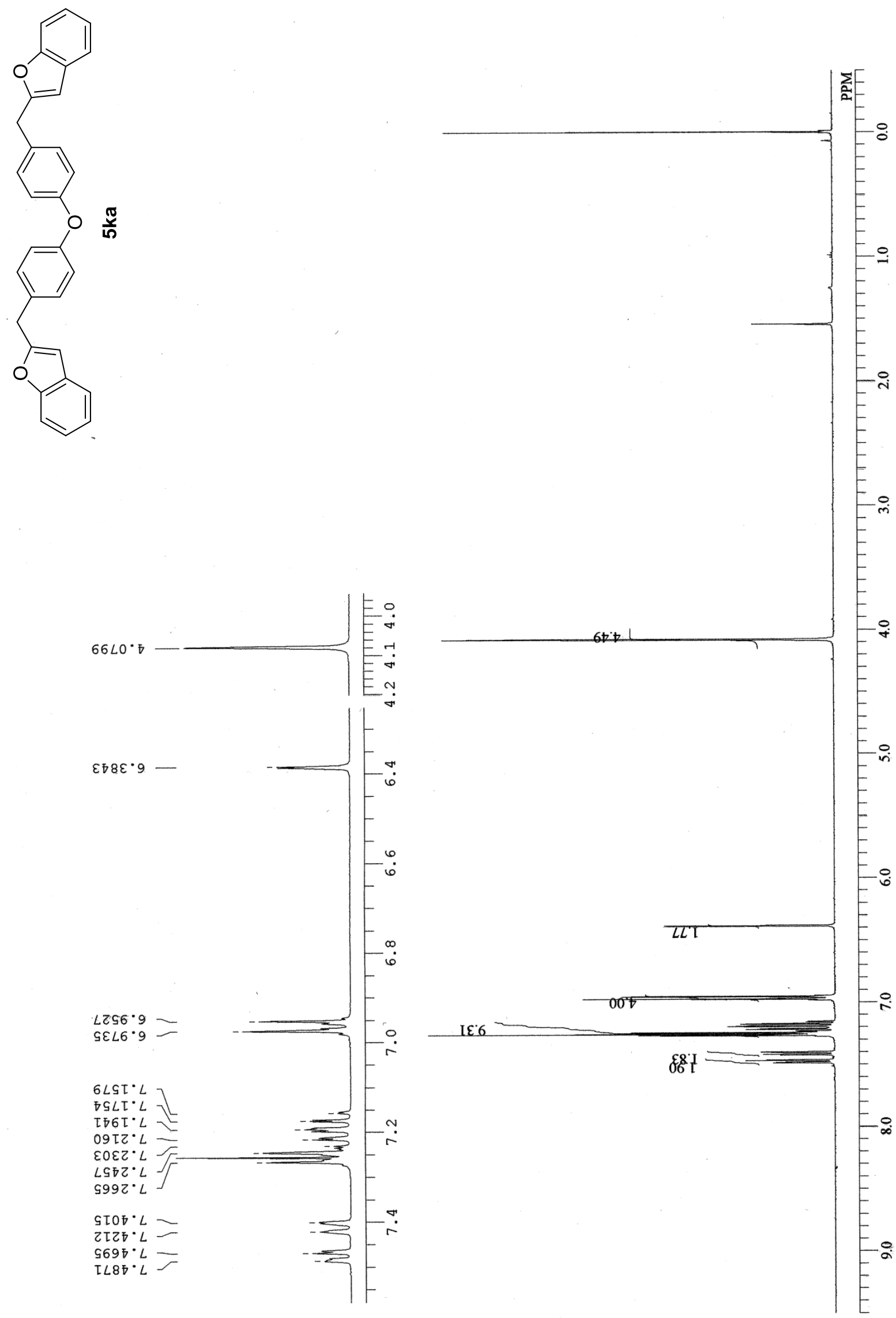

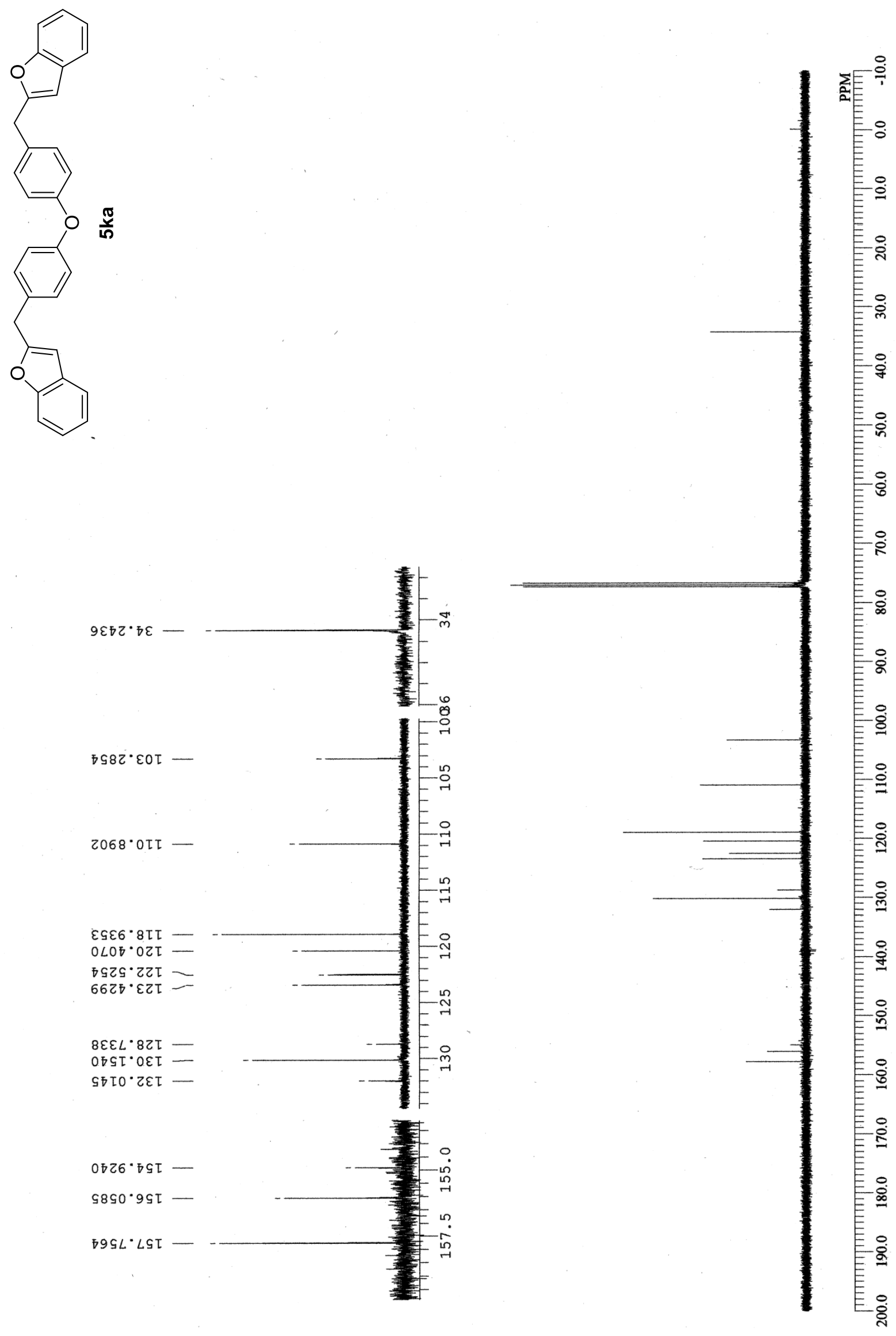


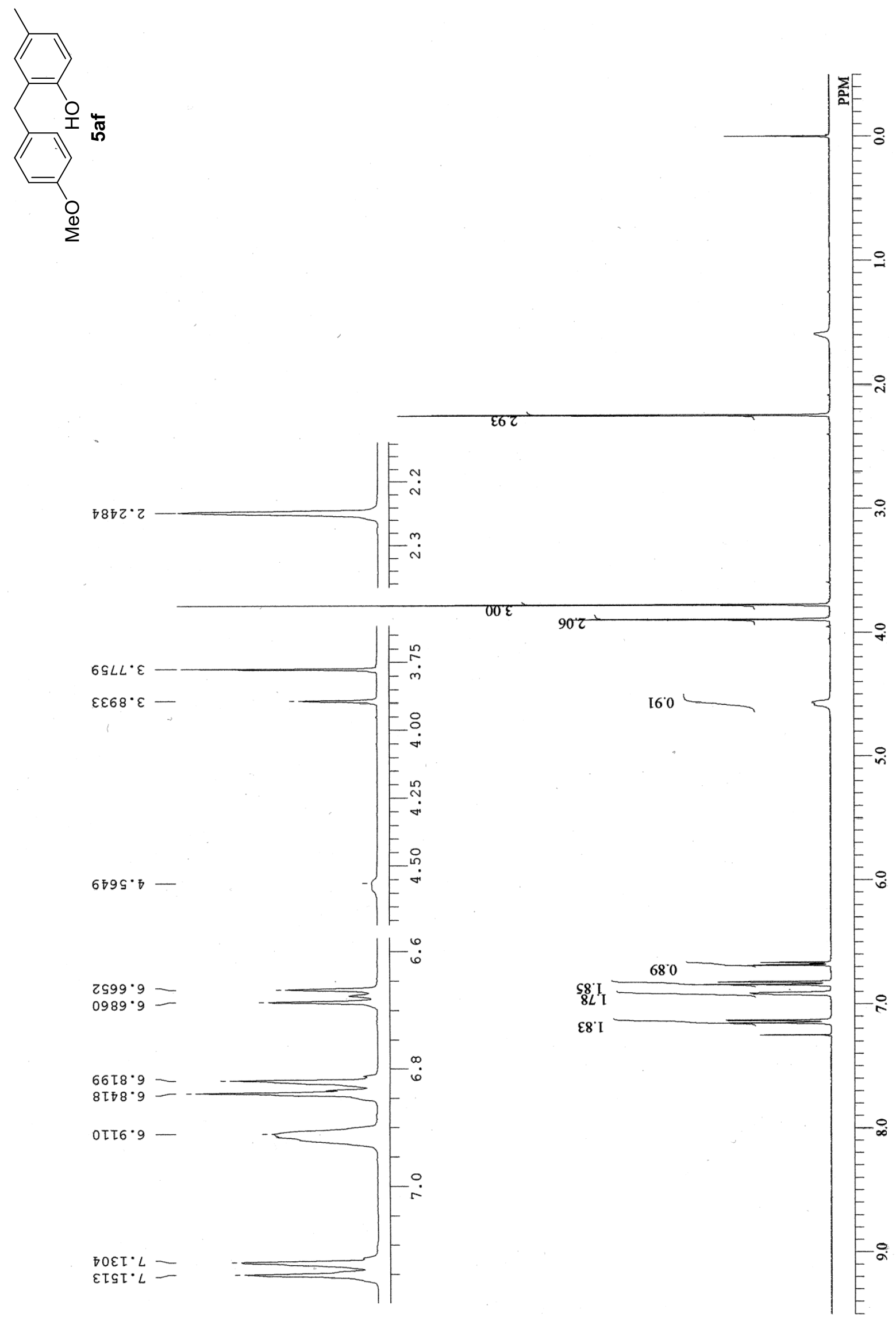



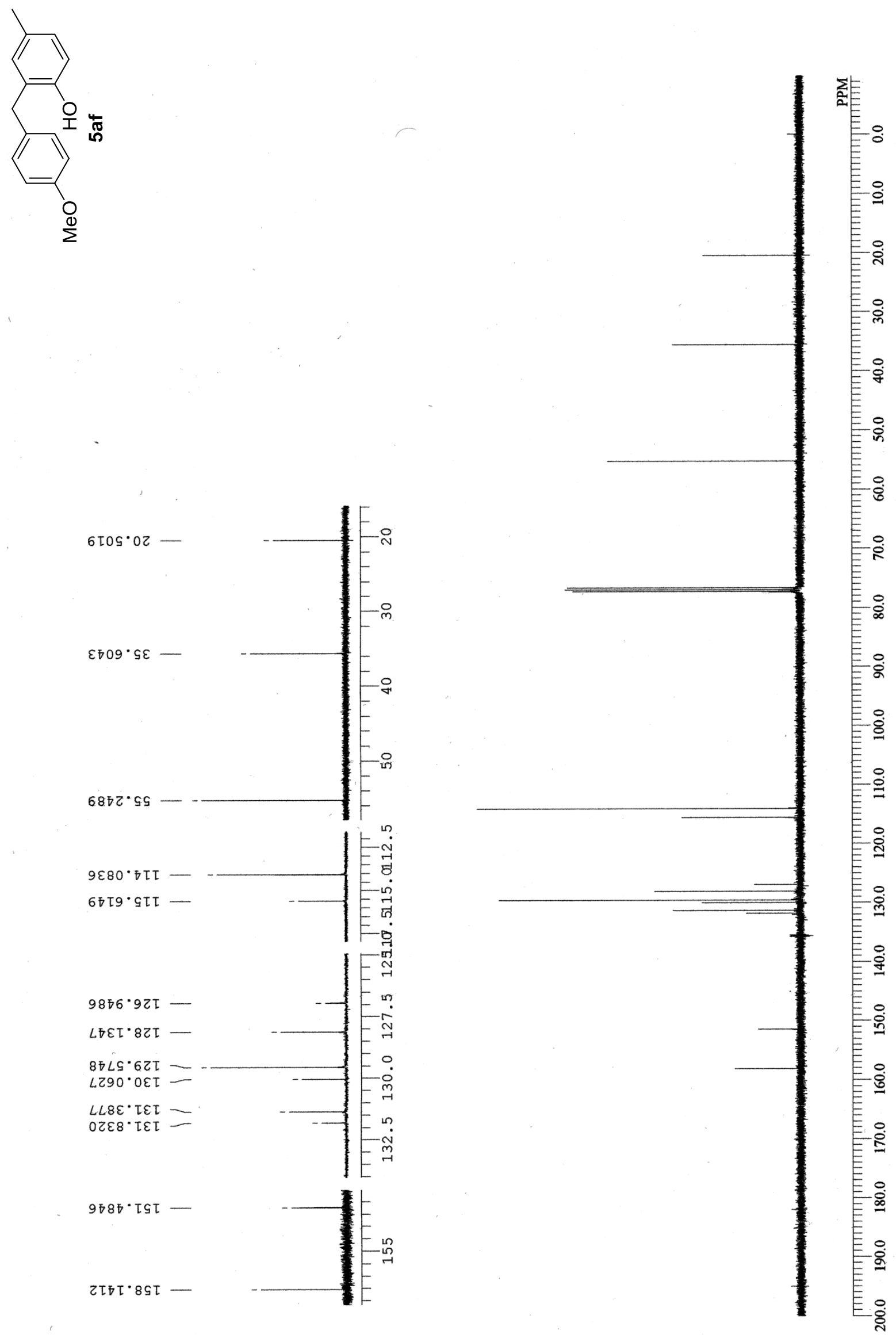

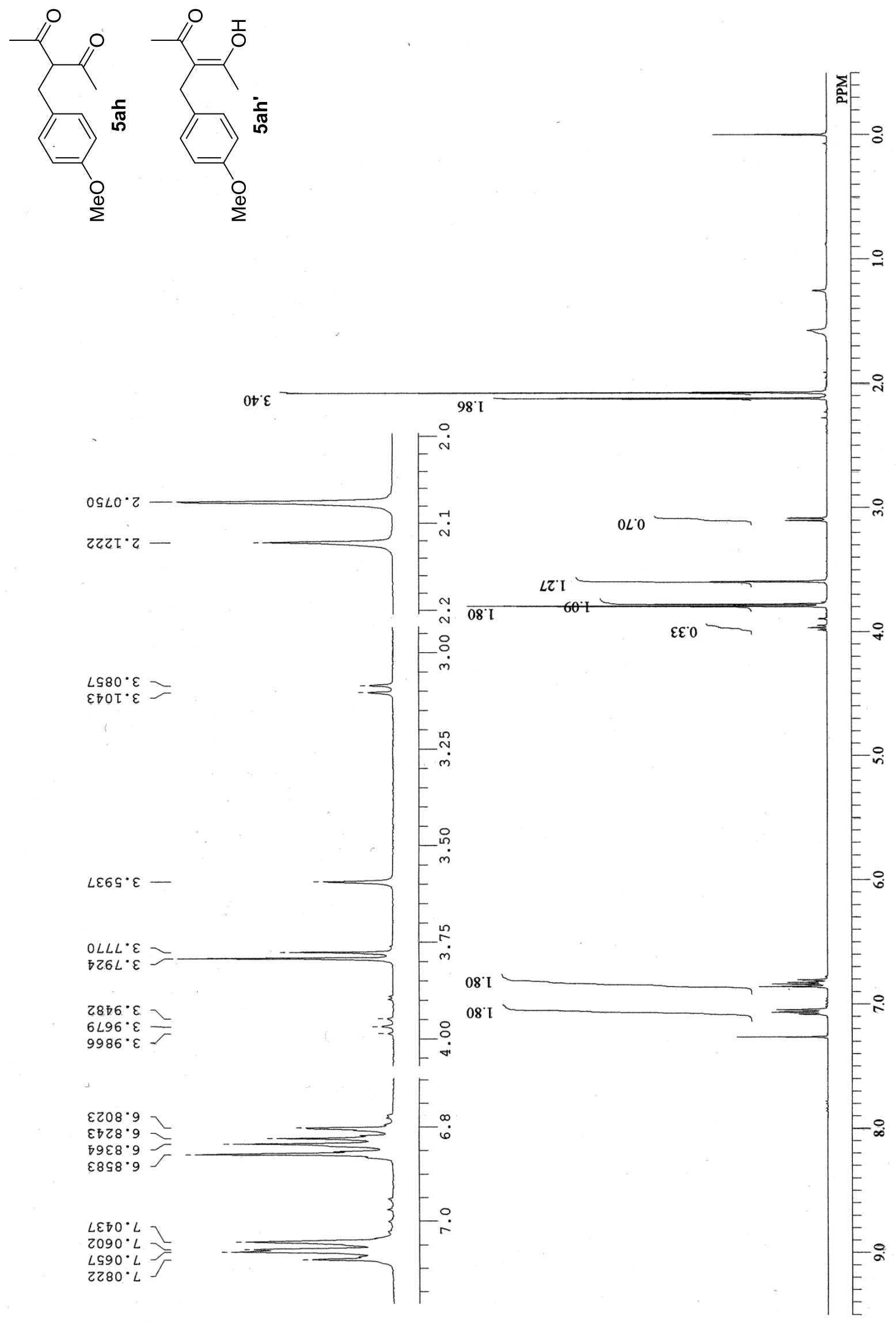

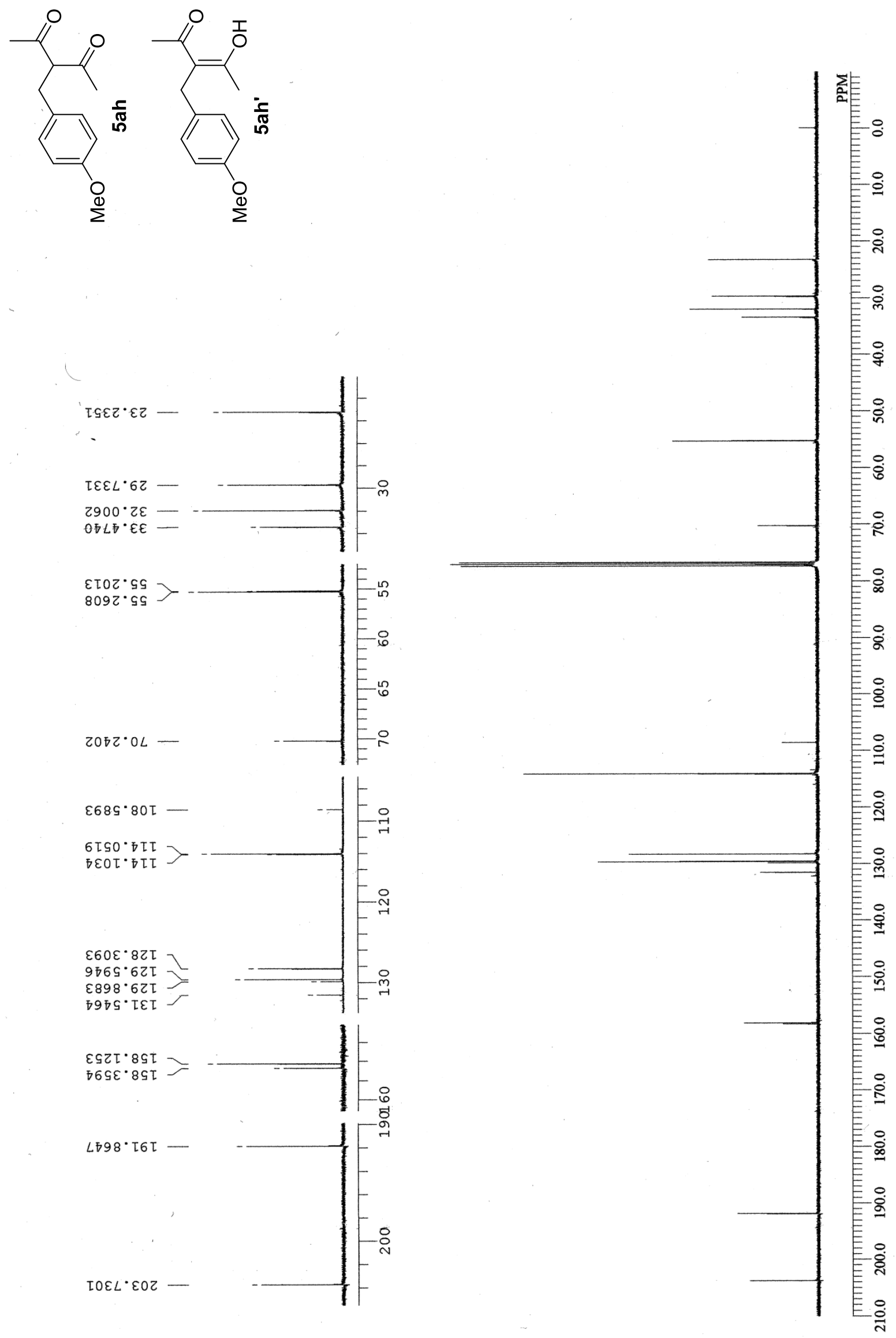

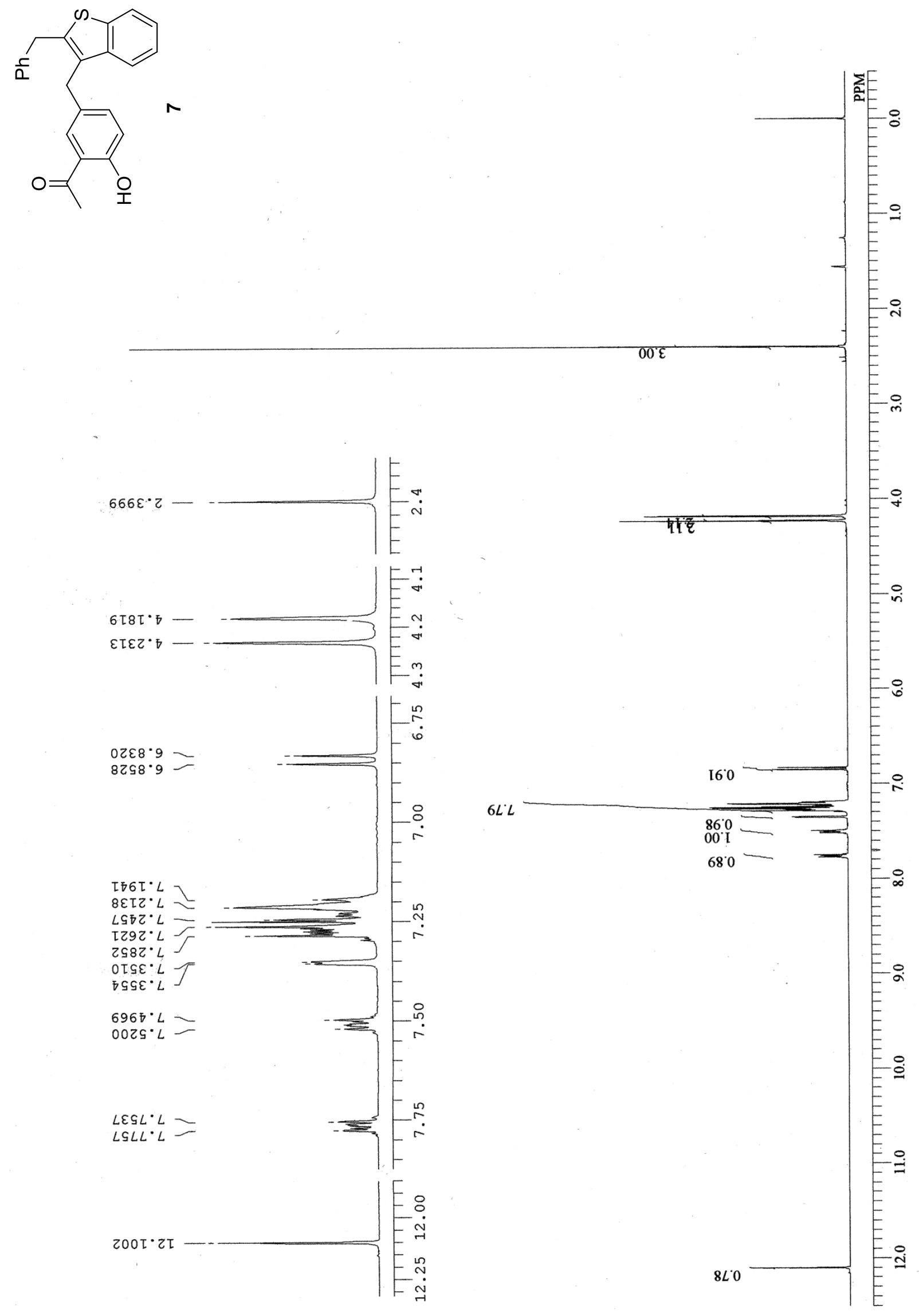

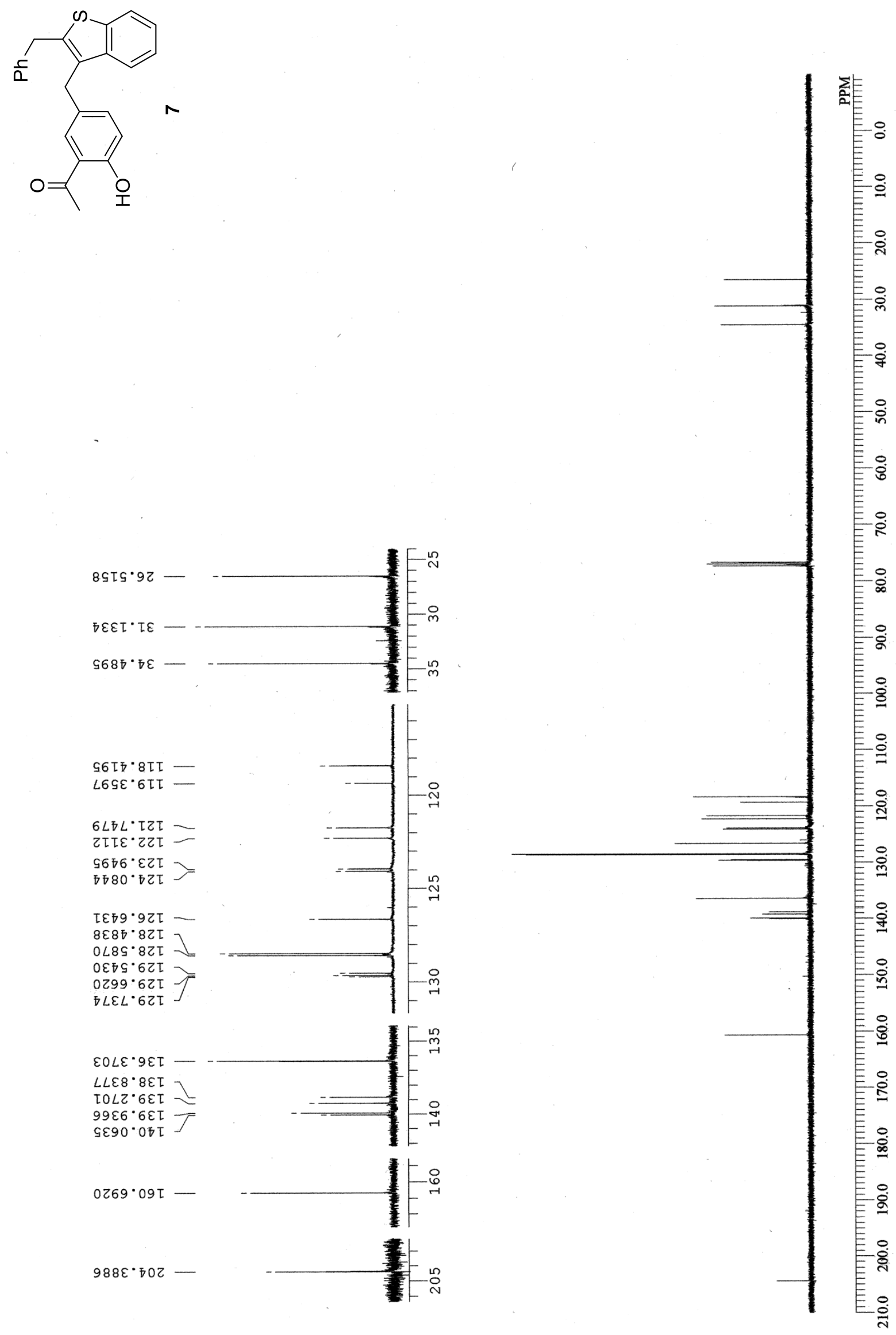
<smiles>COc1ccc(C)cc1C(C)=O</smiles>
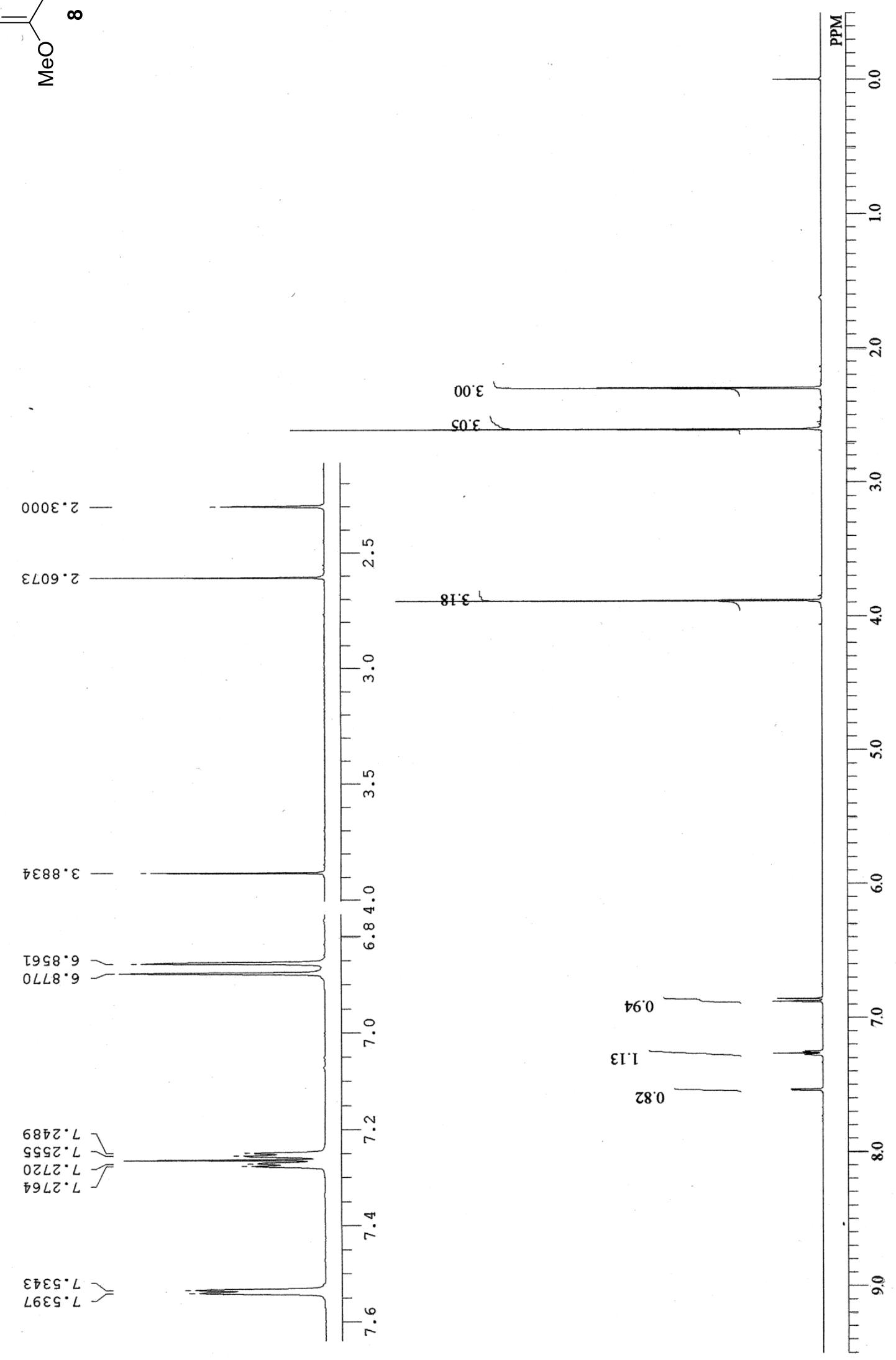
<smiles>COc1ccc(C)cc1C(C)=O</smiles>

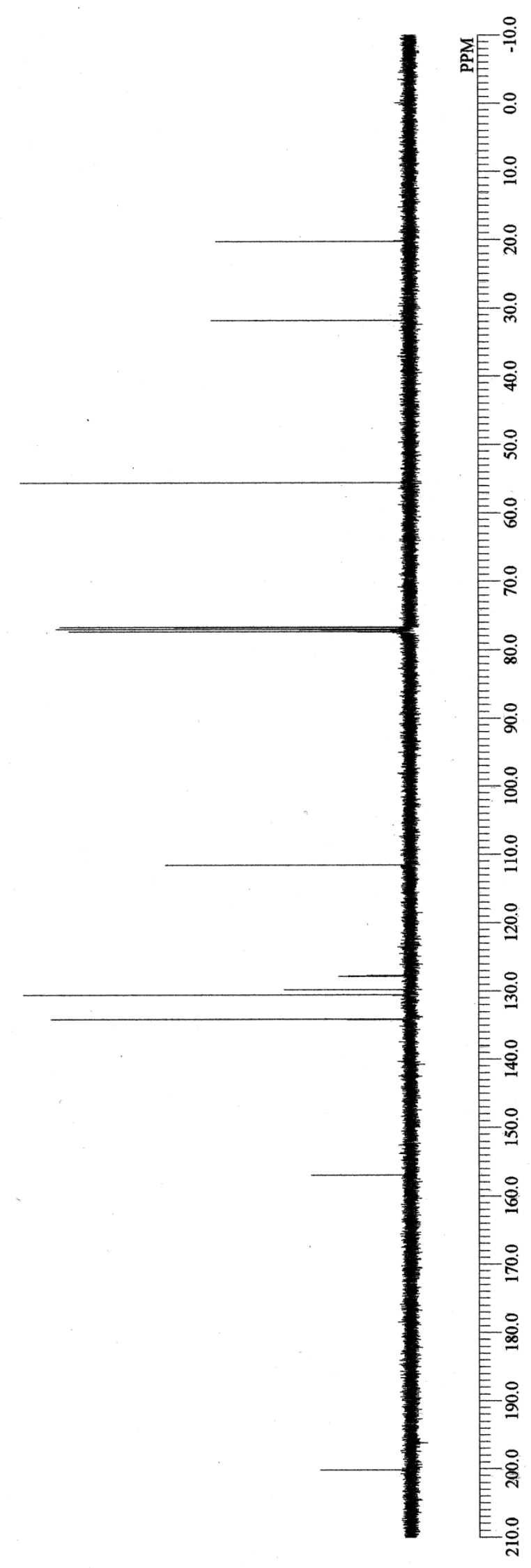



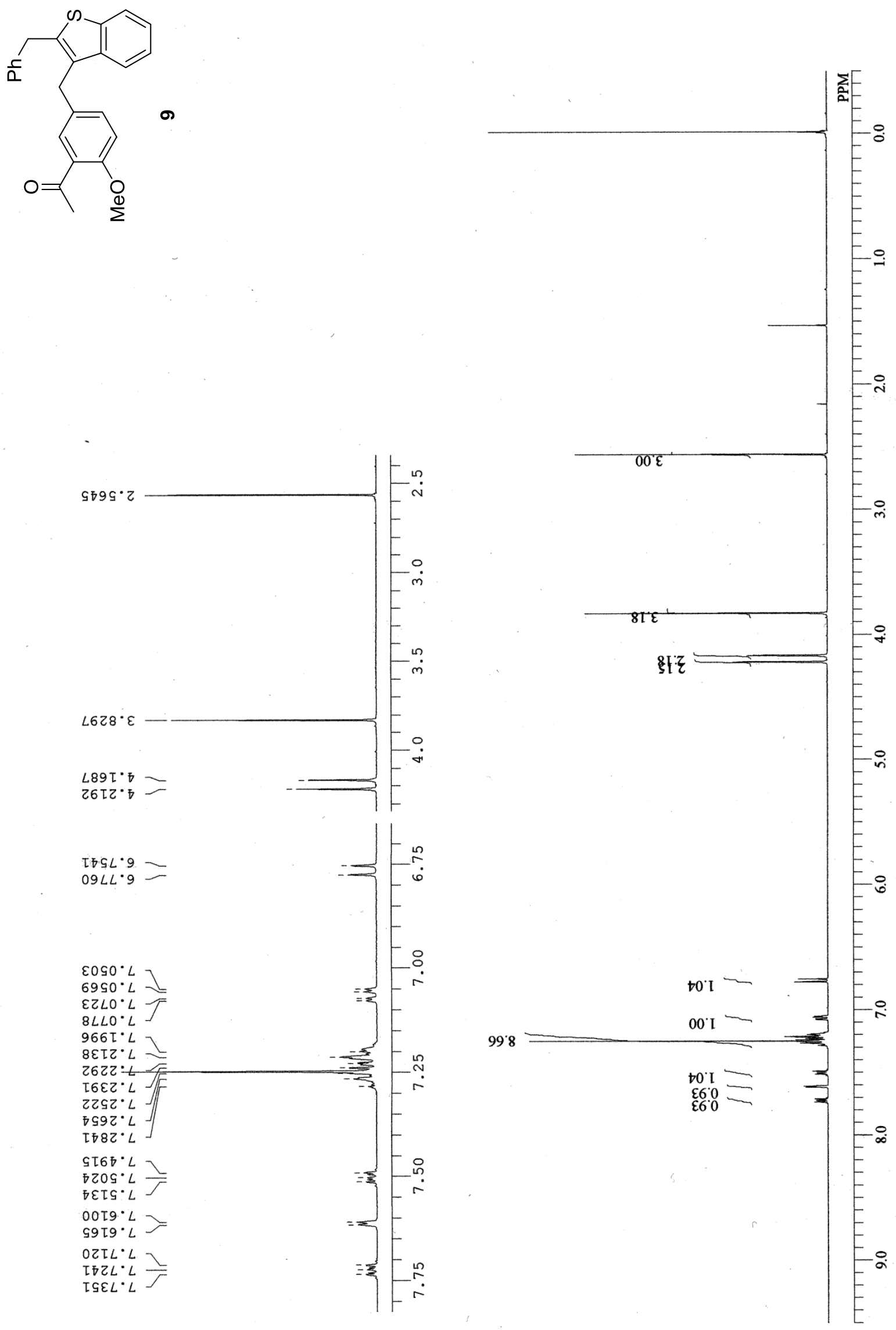
<smiles>[CH2]Cc1oc2ccccc2c1Cc1ccc(OC)c(C(C)=O)c1</smiles>
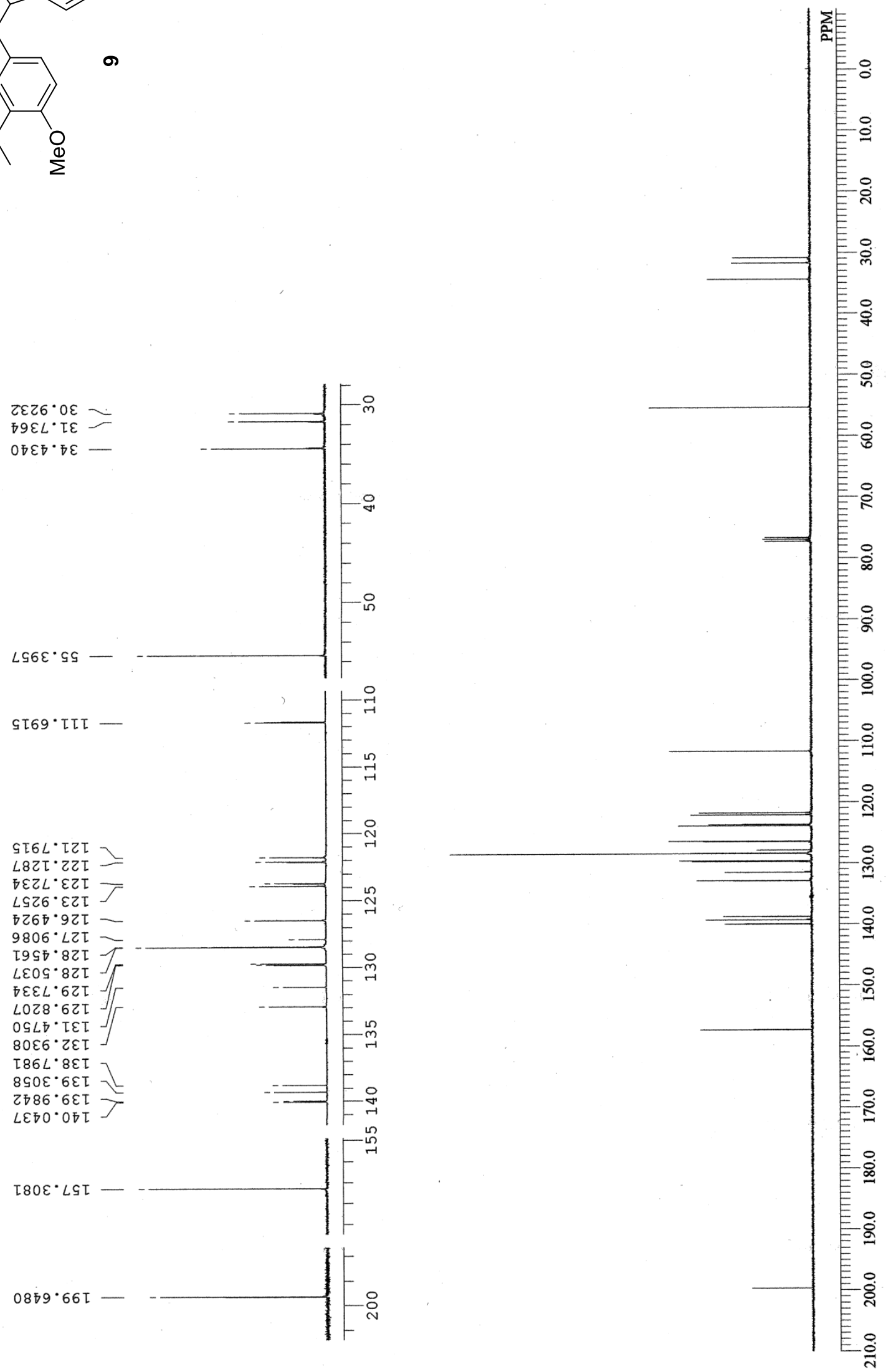